




WOONSPE ITAKIHNA.

solt

\section{PRECEPT UPON PRECEPT:}

TRANSLATED INTO

\section{The Dakota Language.}

BY REV. JOHN B. RENVILLE.

PREPARED FOR THE PRESS

BY EEV. S. E. IIIGGS, D.D., II.D.

REVISED EDITION.

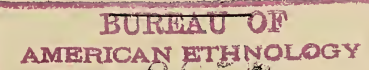

$683 \%$

1807

II B IR A IR Y

AMERTEAN TRACT SOCIETY,

I 50 NASSAU STREET, NEW YORK.

1889. 
p.1024

$$
\begin{array}{r}
1776 \\
1889
\end{array}
$$




\section{TAKU EN UN KIN.}

WOONSPE.

YUMDAPI,

1. Samuel, qa hunku waśte kin $\ldots \ldots \ldots \ldots \ldots \ldots \quad 5$

2. Samuel wokcan cistinna kin............ 9

3. Samuel, qa Wakantanka Koka tawa ........ 13

4. Samuel, qa Dagon wakağapi kin .......... 17

5. Saul wicaśtayatapi kin $\ldots \ldots \ldots \ldots \ldots \ldots \ldots .22$

6. Saul, qa taku śica econ qon ............. 27

7. Dawid tahinca ska awanyake cin.......... 31

8. Dawid, qa candowankiyapi kin........... 34

9. Dawid, qa Golia wicaśta tanka qon ........ 37

10. Dawid, qa wahonkeza kin ............ 43

11. Dawid, qa imnija olidoka kin .......... 47

12. Dawid, qa Saul tawahonkeza kin ......... 53

13. Dawid, qa wahoyapi yuecetupi kin ........ 57

14. Dawid, qa Koka kin Zion Paha akan........ 61

15. Dawid, qa Uriah tawicu qon........... 66

16. Dawid, qa iyopeyapi kin ............ 71

17. Dawid, qa Utuhu can kin............. 76

19. Dawid, qa iyaye cin $\ldots \ldots \ldots \ldots \ldots \ldots . . \ldots 1$

19. Solomon, qa taku waśte kahinig̀e cin ....... 85

20. Solomon, qa Tipi Wakan kin $\ldots \ldots \ldots \ldots \ldots 90$ 
21. Solomon, qa wicaśtayatapi winolinca kin..... 94 22. Solomon, qa wakagiapi kin $\ldots \ldots \ldots \ldots \ldots \ldots 98$ 23. Jeroboam, qa nape śnije qon. .......... 101 24. Elijah, qa kangi kin ................ 104 25. Elijah, qa wiwazica kin................ 108 26. Elijah, qa wahna wośnapi nonpa kin........ 114 27. Elijah, qa magaju kin ............... 120 28. Elijah, qa Eliśa kicopi qon ............. 124 29. Elijah, qa Nabot tawoju kin . . . . . . . . 128 30. Elijah, qa akicita tancan yamni ......... 134 31. Elijah, qa peta canpahmihma kin ........ 137 32. Eliśa, qa wahanksica kin ............. 139 33. Eliśa, qa wicinyanna kin . . . . . . . . . . . 143 34. Israel wicaśtayatapi ehake kin . . . . . . . . 147 35. Hezekiah Wakantanka wacinye ciqon ........ 148 36. Nebukadnezzar, qa wakagapi kin . . . . . . 152 37. Nebukadnezzar wihamna qon ........... 158 38. Belśazzar, qa wicanape wowa qon ......... 162 39. Daniel, qa mnaja otipi kin $\ldots \ldots \ldots \ldots \ldots 166$ 40. Jerusalem ake en ahdi kin ............ 172 Itancan Tawocekiye kin $\ldots \ldots \ldots \ldots \ldots \ldots 177$ Woahope Wikcemna kin ............... 177 Wicayawaśtepi kin............... 179 


\section{WOONSPE ITAKIHNA.}

\section{WOONSPE 1.}

SAMUEL QA HUNKU WAŚTE.

Hokśipidan, wicinyanpidan, Israel oyate kin Kanan makoce kin en token upi, qa taku akipapi he wowapi wakan kin en oyakapi. Jośuwa te cin iyohakam taku akipapi kin ociciyakapi kte.

Unkan hehan tuwe wicaśtayatapi un he. Wakantanka Wicaśtayatapi iyotan kin hee. Jośuwa he wicaśtayatapi un he. Jośuwa he wicaśtayatapi śni; tuka oyate kin token econpi kta Wakantanka cin kin he oyagkiya. Qa Jośuwa te cin iyohakam, ake wanji tokeca Wakantanka woyagkiya.

Śloh otonwe kin en ohna wośnapi wokeya kin heca wan han, qa he en wośna itancan kin ti. Wośna itancan kin Eli eciyapi. Eli he wicaśta waśte hinca. Oyate kin wicota omaka otoiyohi otonwe kin he en wośna ai, qa Wakantanka ohodapi ece.

Oyate wośna ai ece kin he en opeya wicaśta 
wan tawicu nonpa. Tuka he wanakaja, qa he woahtani tanka, hecen dehan tuwedan tawicu nonpa śni.

Tawicu kin unma winohinca waśte, he Hannah eciyapi; tuka Hannah cinca nica. Qa tawicu unma kin he sica, tuka he cinca ota. Winohinca sice ciqon he Hannah ihaha qa heciya; Hannah Wakantanka śicedaka nakaeś cinca nin un ce, eya. Tuka he hecetu śni. Wakantanka Hannah waśtedaka. Winohinca sice cin hena heya eca, Hannah onśika tuktekten on ceya ece.

Ake Hannah Śiloh otonwe kin en $\mathrm{u}$; unkan winohinca unma qon he ake ihaha. Tuka Hannah wośnapi wokeya kin mahen i, qa Wakantanka cekiya. He icunhan Eli wokeya kin timahen yanka. Unkan Hannah ceya un kin heon iśta kin popo, qa ho onśiic̣iya wocekiye eya. Hannah iśta popo kin he Eli wanyaka, unkan miniśa ota yatkan on hececa kecin ; qa, Tokeca e miniśa ota datkan he, eciya. Wicota ehna heciya heon Hannah nina iśteca. Tuka Hannah iś itkom ho onśiic̣iya ayupte ça, miniśa mdatke śni ce, eya ; iyomakiśice cin on Wakantanka cekiya waun ce, eya.

Hehan Eli he nahon kin on ihnahna okiye ça heya; Tokin Wakantanka taku iceyakiye cin aniyupten, eciya.

Hannah taku icekiya he. Hokśiyopa wanji yuhe kta he icekiya; qa wanji yuhe cinhan Wa- 
kantanka qu, qa iye icaliye ça oie kin yaotanin kta he icekiya.

Hehan Hannah Eli ihnahna okiye cin on iyuśkin, qa iśtamnihanpe kpakinte ça, tiyata ki, qa nina iyuśkiniçiya. Iho, micinca, taku wanjidan on wicocante waśte, wocekiye kin hee. Tohan iyoniciśicapi, qa iś waniyazankapi, qa iś wowi-

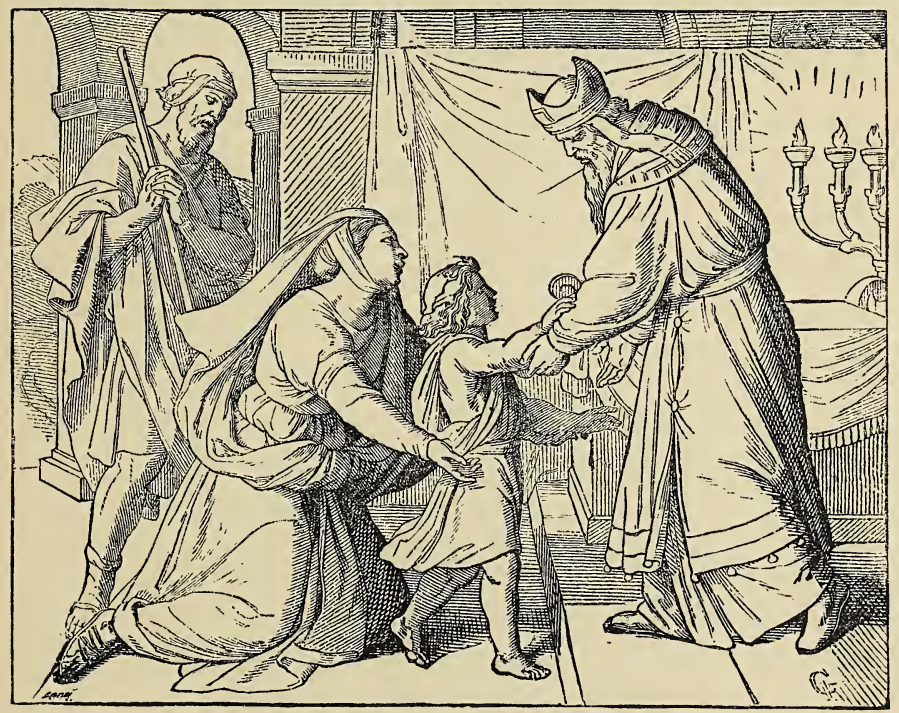

SAMUEL HOKŚIYOPA KIN.

śtece wanji en aniupi kinhan, nina Wakantanka cekiya po.

Hehan Hannah Śiloh etanhan kihde ça Kanan ekta ki. Unkan hokśin yuha. Hokśidan wan yu- 
he ça Samuel eya caśton, Wakantanka icekiye ciqon hee. Unkan tohanyan Samuel hokśiyopa kin hehanyan Hannah Śloh ekta ye śni. Tuka wanna hokśidan kin waniyetu yamni topa ecetu qehan, hehan Śiloh ekta kici ya.

Hannah hokśin yuhe cinhan token Wakantanka iwahoye ciqon he akiktonje śni. Cinca kin nina waśtekidaka heon etanhan Wakantanka en icağe cinhan waśte, qa oie kin yaotanin kte cin he Hannah cin. Hecen wośna itancan kin Eli he qu; qa heciya; Eya winolinca wan wośnapi wokeya kin en cekiya hi wandake ciqon he miye ce, eya. Hokśiyopa kin de Wakantanka maqu kta icewakiya. Unkan maqu ce. Tuka ake iye icaliye cinhan waśte kta. He wacin heon etanhan duhe kta e cicahi ce, eya.

Hecen Eli hokśidan qon yuha. Hehan Hannah iśnana kihde ça hihnaku kin ekta ki. Qa Wakantanka taku icekiya, unkan qu kin, heon yatan qa woyatan odowan wan ahiyaya.

Unkan ake cinca wanhdag hi ece he. Han, omaka otoiyohi wanhdag hi, qa wokoyake kahi ece. Hehan oyate kin token oihduzeyapi kin he iyecen Samuel ihduza; miniliuha zimzipedan kin heca koyake ça tawokoyake kin hanska, siha kin ekta aiyahdeya koyaka.

Unkan Wakantanka Taniya Wakan kin Samuel cante kin ekta ehnaka. Heon tohtani kin nina 
waśtedaka. Qa tohan wokeya kin ohna wośnapi eca, wośna itancan qa oyate kin nina Wakantanka yatanpi nalion, qa wanyake cin hena on sanpa waśte icah aya. Qa oyate kin Samuel waśtedakapi. Hannah cinca wanhdag hi, qa hena nalion eca on sanpa iyuśkin ece.

Tho, micinca, niś eya niwaśtepi kinhan, heon nihunkakepi kin iyuśkin wicayayapi kta, qa iyotan Jesus Itancan kin iyokipi kta, qa nakun mahipiya oyate kin.

Samuel token hokśiyopa waśte kin he iyecen niś Wakantanka en woonspe ode po.

\section{WOONSPE 2.}

SAMUEL WOKCAN CISTINNA KIN.

Samuel Eli kici Śiloh en ti nayalionpi qon. Wośnapi wokeya kin ohna tipi śni, tuka icahda wakeya otipi.

Eli cinca nonpa; qa napin wanna kośkapi, qa wośnapi itancan kin hecapi. Unkan Eli wicaśta waśte kin heon iś eya cinca kin napin iyececapi kecannipi nace. Tuka napin wicaśta ohan śica hecapi; qa Wakantanka ohodapi śni, qa iye takı imağağaiçiyapi, qa iyokipipi ecedan econpi.

Hecen Eli iye wicaśta waśte, tuka cinca kin 
napin śicapi. Eli cinca kin hececapi, tuka nina anawicapte śni kin heon Eli tanyan econ śni.

Wicaśta owasin cinca taku śica econ kinhan iyopekiye kta. Ecin tuwe hecon śni kinhan okinni cinca wan tehinda eśta Wakanśica ti kin en iyohpaye cinhan he tehike.

Iho, heon etanhan, micinca, niś eya tohan taku sica ecanonpi kinhan, nihunkakepi kin nicapsinpsintapi kte cin he hecetu.

Eli cinca taku śica econpi kin hena nalion eca on wahokonwicakiye c̣a hewicakiya ; Micinkśi Wakantanka iyokipiyayapi śni ce. Okinni canniyeniyanpi kinhan kakiśniyanpi kta ce, ewicakiya keś, nakun anag̉optanpi śni.

Unkan wicaśta wan waśte Eli en hi qa heciya ; Wakantanka canteptanyan ce; tokata anpetu wan en nicinca napin wicaktepi kta ce, eya.

Eli he nahon kin on nina icanśica. Eli cinca nina wahokonwicakiye śni kin heon Wakantanka iyokipi śni; tuka Eli iye kin wicaśta waśte kin heon Wakantanka waśtedaka.

Eli qa Samuel kinunkankiya ohehdepi. Unkan hanyetu wan en wanna iwankapi kin hehantu, Samuel wicaho nahon; Samuel, Samuel, eya. Unkan iś, De miye ce, eya. Eli hee kecin. Samuel hokśidan waśte nakaeś, Eli iyokipiye kte cin he ape wiyeya un, heon kohanna ekta i qa; Taku he yaka he, eya. Tuka Eli iś ayupte ça, He miye 
śni ce, eya. Hecen ake Samuel iye tohe kin en kiwanka.

Tuka ake iecadan tuwe kipan qa, Samuel, eya. Ake Eli ekta i, qa, He niye mayakipan he, eciya. Tuka Eli iś ayupte ẹa, Micinkśi he miye śni ce, ekta kiwanka wo, eciya. Qa ake Samuel ekta kiwanka. Tuka ake ecahankeya tuwe, Samuel, eya. Hantu qeś Eli hee linca kecin, qa Eli ekta i qa; Ho de miye ce; he niye miyeco ce, eya. Samuel tuwe kipan ece kin Eli he tuwe kin sdonya. Micinkśi he tuwe kin sdonyaya he. Itancan kin hee ce, eya. Hehan Eli Samuel heciya; Ekta kiwanka wo, qa ake eye cinhan, Itancan nitaokiye niho kin nahion ce, eya wo, eciya.

Ake Samuel ekta kiwanke cin iecadan, Itancan kin ikiyedan hinajin, qa; Samuel, Samuel, eya. Hehan Samuel ayupte ça ; Itancan, niho kin nitaokiye nahon ce, eya. He itokam tohinni Wakantanka Samuel okíye śni; heon etanhan taku nahon kta cantokpani; tuka hehan nahon, unkan Eli on etanhan taku wanji iyokiśica nahion.

Wakantanka Samuel heciya; Eli cinca kin śicapi, tuka nina wahokonwicakiye śni kin heon etanhan, ito, kohan kakiświcawaye kta ce, eya. Wakantanka Samuel taku eciye cin yaśtan qehan, ake Samuel ecen kiwanka. Samuel Wakantanka taku eciye qon he Eli okiciyake kte cin itonpa.

Ihanhianna Samuel kikta, tuka Eli yanke cin 
en i śni; wokeya kin mahen wicohan hena econ ece qon hena econ. Hehan Eli Samuel kico, qa hinhan Wakantanka taku okiyake cin he takudan anahbe śni okiciyake kta icekiya. Unkan Wakantanka taku Samuel eciye cin owasin Eli okiyaka.

Hehan Eli he nahon kin on nina icanśica, cinca kin śicapi tuka nina wahokonwicakiye śni, qa Wakantanka iyokipiye śni kin heon. Hehan Eli ho onśiiçiya ayupte ça heya; Iye Itancan kin token iyokipi kinhan ecen econ nunwe, eya. Eli Wakantanka nina waśtedaka, tuka heceedan on tanyan econ śni.

He iyohakam Wakantanka taku econ kta eca, itokam Samuel okiyake ca; Samuel iś iyoopta oyate kin owicakiyaka ece. Qa taku oyake cin owasin iyecetu ece, hecen hetanhan Samuel Waayata heca.

Samuel taku oyaka eca he oyate kin woanagoptan yapi. Hèca keś nakun apa iyowinye śni taku sica econpi. Śiloh en oyate unpi kin Wakantanka iyokipiyapi sni, heon kohanna iś eya Wakantanka iyopewicaye kta, Eli cinca kin hena om. Tuka Wakantanka Samuel waśtedaka, hecen Samuel iyuśkiniçiya.

Iho, micinca, tokin miś Wakantanka waśtemadake çeś, ecannipi kinhan, niś waśteyadakapi, qa taku iyokipi eceedan ayakitapi kinhan, hecen waśtenida kta. Iye Wakantanka heya; Tona 
SAMUEL QA WAKAN'TANKA KOKA 'TAWA. 13 waśtemadakapi kin miś eya waśtewicawakida ce. Samuel hokśiyopa waśte kin, niś iyecen Wakantanka nina cekiya po.

\section{WOONSPE 3.}

SAMUEL QA WAKANTANKA KOKA TAWA.

Wakantanka Eli cinca kin token kakiświcaye cin he nayalionpi kta. Tuka tokaheya oyate wan Pilistim ewicakiyapi kin he owicamdake kta.

Pilistim oyate kin Kanan makoce kin ahankeya tonwanyanpi. Tuka oyate kin he śicapi, qa wakaggapi ohodapi, qa Israel oyate kin tokawicayapi, qa taku yuhapi ko wicakipi ece. Tuka Israel oyate Wakantanka awanwicayaka heon etanhan Pilistim kin kiunniwicayapi śni; tuka wanna Israel Wakantanka iyokipiyapi śni.

Anpetu wan Pilistim wicota zuya u, qa ahiti. Israel he nakilionpi, unkan iś eya piiçiyapi, qa itkowicakipapi qa om ocitaninyan etipi. Qa ihanhanna hehan om kicizapi. Unkan unma tukte ohiyapi he. Pilistim e ohiyapi. Israel wanna Wakantanka ówicakiye śni, heon etanhan nina yuśinyayapi qa nakipapi.

Israel wahtanipi kin on iyopeiçiyapi, qa Wakantanka nina cekiyapi kta iyececapi, tuka nakun heconpi śni. Qa heyapi; Wakantanka Koka ta- 
wa kin he huweya miye; he den yanka unkanś unkiye ohiunyanpi kta tuka ce, eyapi.

Koka kin he oyanke kaġapi, qa akapatanhan kin owancaya mazaskazi kin heca. Unkan he tuktekten Wakantanka malipiya śapa wan akahpa ece. Tuka Koka kin he oyate niwicaye kte cin hee śni. Wakantanka he oyate niwicaye kte cin hee.

Hehan Israel Śiloh ekta tuwe yeśipi, qa Koka qon he iye tipi kin en wicakaupi kta cinpi. Unkan Eli cinca qon heepi Koka qon wanna aupi. Israel he wanyakapi kin on nina iyuśkinpi qa iyakiśapi. Nina iyakiśapi kin hecen tehan tanhan tanin. Qa Pilistim he nahonpi qa heyapi; He taku e on hecen Israel iyakiśapi hwo, eyapi. Unkan hehan nahonpi.

Wakantanka Koka tawa qon he Israel wicakicicaupi ce, eyapi, Pilistim he nahonpi, unkan nina wikopapi, qa heyapi ; Wakantanka Paro taoyate om awihnuniwicaye ciqon ake dehan Israel ówicakiya ehantanhan unkiś eya iyecen awiunhnunipi kta nace, hecen token unhanpi kta he, eyapi.

Tuka akeś heyapi ; Ho miye, heca eśta ipajinyan token waunśakapi wicunkizapi kte, eyapi. Qa ake om kicizapi he icunhan Koka kin wośna itancanpi kin en ahipi.

Tuka Wakantanka Israel ówicakiye śni; he- 
SAMUEL QA WAKANTANKA KOKA TAWA. 15

cen iyayewicayapi qa ota wicaktepi; qa Eli cinca kin napin nakun wicaktepi; Wakantanka hecen eye ciqon iyecetu.

Hehan Koka kin he Pilistim icupi. He icupi kin on nina iyuśkinpi, qa iye totonwepi kin ekta akipi.

Eli iś okicize kin ekta hi śni. Śloh ekta ti kin e heciya yanka. Koka kin he okicize ekta eyayapi kin heon nina icanteśica, qa token econpi kte cin he nalion cantokpani yanka. Śloh en conkaśke tiyopa kin hen tewanhankan oyankeye ça he akan yanka.

Hehan okicize kin eciyatanhan wanji wahośi u. Watuśekśeca pa kin aihdada, qa tawokoyake nakun aihdupote ça, canku ohna dus u. Wicota wicaktepi hośi u kin heon hecon. He ehan waśihdapi eca hecen econpi ece. Wicaśta kin he hecen u, tuka Eli wanna wicahinca, qa iśtağonge nakaeś wanyake śni; ecen wicaśta qon he Śloh otonwe kin ehna ahioyaka. Hehan oyate kin nina wicaceye cin Eli iś hehan nahon, qa wiwanga.

Unkan wicaśta qon he ayupte ca heya; Kicizapi tuka Israel iyayewicayapi, qa wicota wicaktepi, qa nicinca kin napin nakun wicaktepi ce; qa Koka qon he nakun Pilistim icupi ce, eya.

Eli he nalion kin on nina iyokiśica; qa he icunhan tehan wankantuya yanka, tuka iheyatanhan takudan kaicinyan yanke śni; hecen heciyatanhan ohinhpaye ça tahu paksa. 


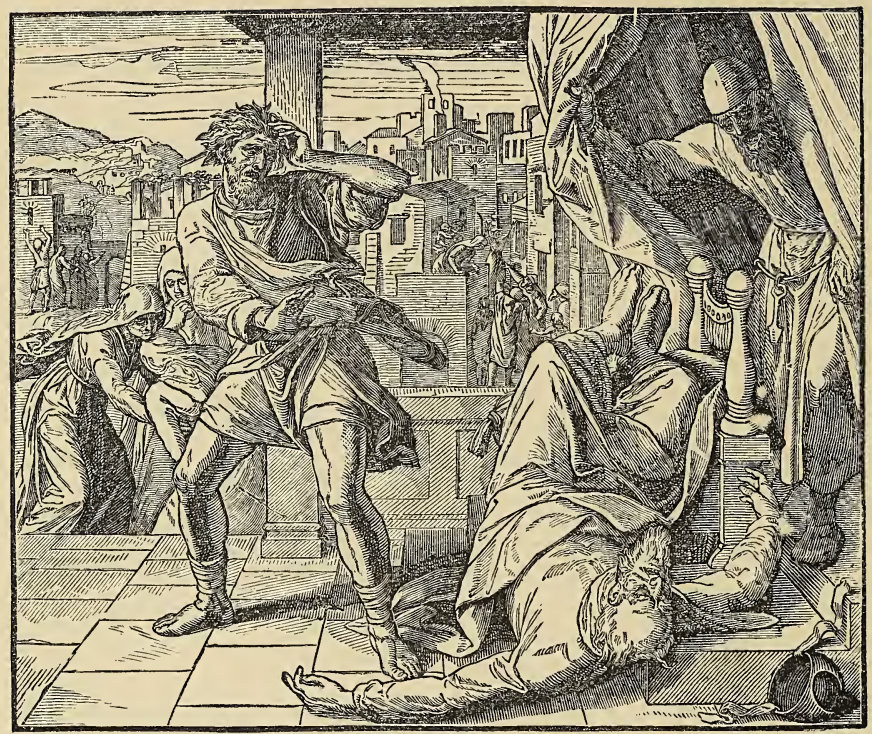

ELI TE CIN.

Hehan Samuel Eli ța nalion kin heon nina iyokiśica. Unkan Eli naggi kin toki un he. Eli Wakantanka nina waśtedaka, hecen wanna ti kin ekta un. Wakantanka Eli wahtani kin owasin kicicajuju, țe śni itokam.

Unkan Eli cinca kin iś nağipi kin toki yapi he. Okpaza wiconte ekta iyayapi. Ecin walitanipi tuka Wakantanka wicakicicajuju śni ecen tapi. 
SAMUEL, QA D.GON WAKAGAPI KIN.

\section{WOONSPE 4.}

SAMUEL, QA DAGON WAKAGAPI KIN.

Pilistim oyate kin Koka qon he akipi kin he nina iyokipiiciyapi. Wakantanka eceedan wakan qa iyotan kin Pilistim he sdonyapi, tuka heconpi.

Unkan iye totonwepi kin en wakağapi tipi wan hdepi, qa Koka akipi qon he wakağapi tipi kin en ehnakapi. Iye wakagapi wan kağapi, wicaśta iyecen okağapi, qa Dagon eya caśtonpi; qa wakağapi tipi kin en oyanke wan tehanwankantuya kicagapi, qa he en hdepi, qa he nina ohokidapi.

Hehan ihanlianna qehan wakaggapi tipi kin wanhlag ipi. Tuka iye wakagiapi tawapi qon he hinlipaye ca Wakantanka Koka tawa kin itokam ohdapśinyan wanka wauhdakapi.

Unkan he tuwe Dagon hecen elipeya he. Wakantanka hee hecon. Wakaġapi kin hena taku śni, tuka ohodapi kin he sdonyapi cin heon etanhan hecon.

Tuka Pilistim Wakantanka hee liinca hecon kecinpi śni; qa ake nakun wakag̉api tawapi qon Koka kin icahda ehdepi. Qa ihanhanna qehan ake wanhdag ipi. Tuka ake nakun Dagon Koka qon itokam hdilipaye ça, hehan tahu papśun, qa isto 


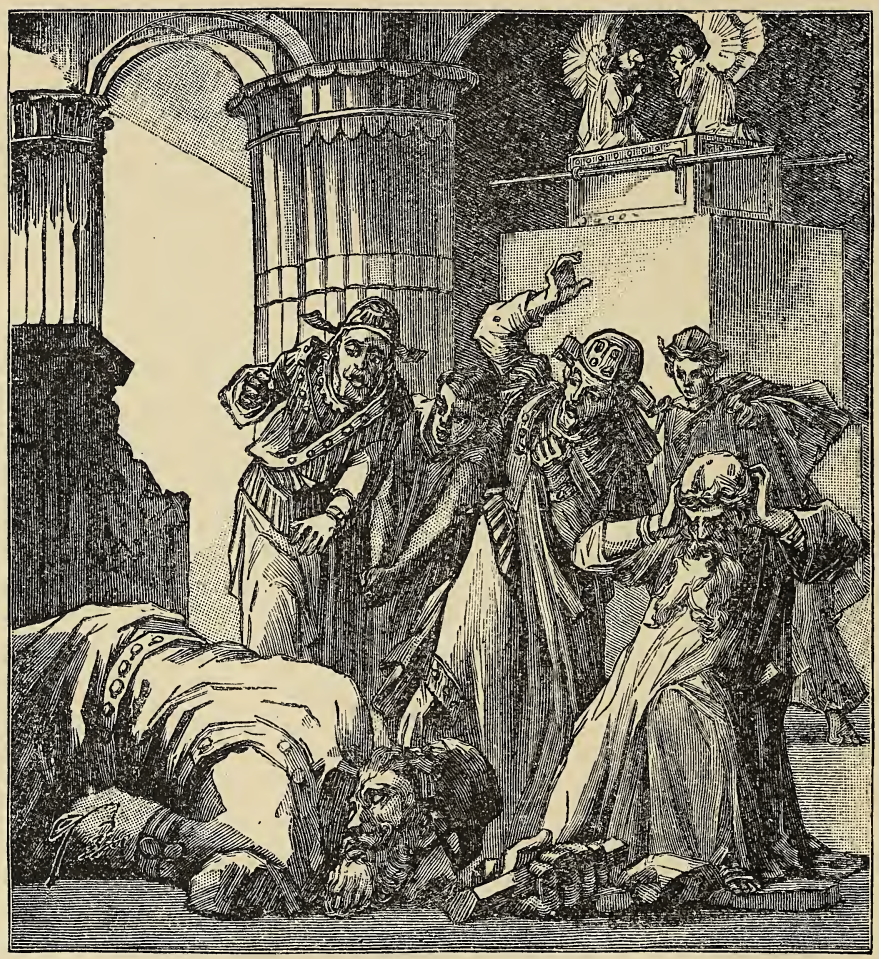

DAGON WAKAGAPI KIN.

napin nakun paksa wanka wanhdakapi; unkan he nina icanteśicapi.

Hehan ake Wakantanka Pilistim oyate kin wowayazan iyahdewicaya. Qa wanna nina wayazankapi kin hehan hekiciyapi; Koka kin de otonwe kin den tehan yanke kta iyecece śni ce, eyapi; qa otonwe tokanta ayapi. Tuka nakun otonwe en 
aipi qon iś eya nina wawicayazanke ça nina wicața. Hecen iś eya otonwe tokan ayapi.

Unkan otonwe wan en ayapi qon he nina kokipapi qa heyapi ; Koka qon hee wanna den aupi ce. Unkiś eya wanna ake nina unțapi kta ce, eyapi. En aipi kin ecahankeya iś eya wawicayazanke ça wicațe ça wicaceya.

Koka kin he yuhapi kin heon Wakantanka wayazanke wicaya, tuka nakun nahanlin Wakantanka hee linca hena hecon kecinpi śni.

Hehan unhanketa Koka kin he token Israel tipi kin ekta ayapi kta he ihdukcanpi. Unkan kaken ihdukcanpi qa heyapi; Koka kin canpahmihma hu nonpa kin heca wanji en unkohnakapi, qa hehan pte wiye nom tohinni heca unpi śni kin he unwicunkiyapi kta ce; qa ptejicadan kin napin den onatag wicunhdepi kta ce; qa pte kin tuwedan wicakaliape kte śni, hecen token econpi kte cinhan wanunyakapi kta.

Qa Israel Wakantanka tawapi kin heceedan iyotan kinhan, tokeśta pte kin owotanna yewicaye ca ecen Koka kin Israel wicakai kta. Qa Koka kin de unhapi kin on etanhan wayazanke unyanpi hecinhan, hehan tanyan sdonunyanpi kta ce, eyapi.

Pilistim hecen wihdukcanpi, qa hdustanpi hehan heyapi ; Wakantanka Paro taoyate om kakiświcaya, tuka iyowinyanpi śni qon; unkiye qe he iyecen econkonpi kte śni ce, eyapi. 
Unkan token ihdukcanpi qon iyecen econpi.

Hehan Wakantanka wowapetokeca wan sdonyewicaya. Pte kin tuwedan awicaye śni, tuka Wakantanka iye owotanna yewicaya. Canku kin ohna katinyan yapi qa tohinni anawam iyayapi śni, owotanna hoton yapi. Pilistim wanjikji ihakam yapi he $\mathrm{i}$ awanyag ayapi.

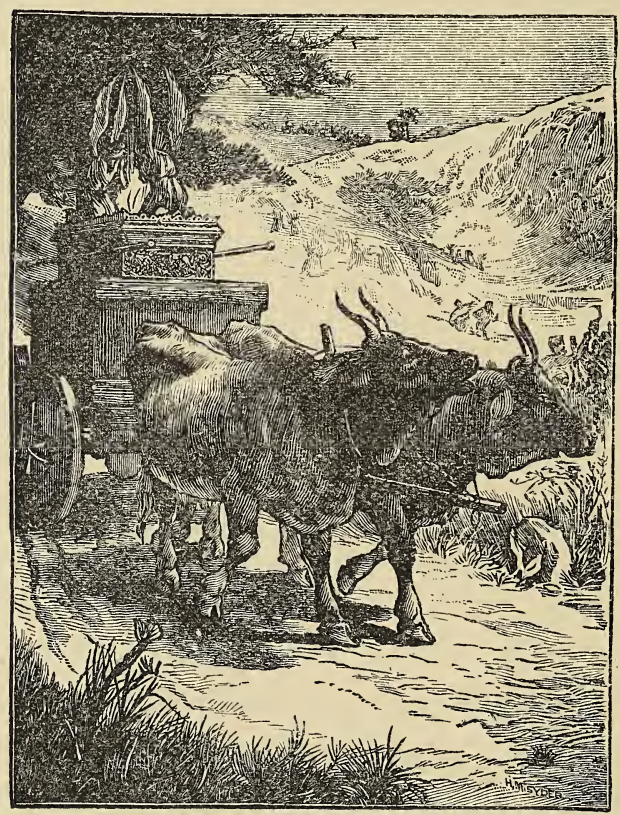

PTEWANUNYANPI KOKA KIN YUSDOHANPI.

Israel etanhan wanjikji maggata woksapi icunhan ptewanunyanpi kin Koka qon hee aupi wanyakapi, qa nina iyuśkinpi. Unkan ptewanunyan- 
pi qon magga kin ehna inyan wan tanka yanke cin he icahda inajinpi.

Wakantanka he Koka kin ake wicakahdi kin he Israel sdonkiyapi, heon etanhan Wakantanka wopida eciyapi, qa wakiyuśnapi cinpi. Hehan Koka qon he icupi, qa inyan tanka kin he akan ehnakapi, qa canpahmihma qon kaksaksapi qa on cetipi, qa ptewanunyanpi qon napin wicaktepi, qa Wakantanka wakiyuśnapi.

Pilistim etanhan wanjikji iawanyag aupi qon heepi icicawin kipi, qa taku wapetokeca wanyakapi kin on yuśinyayapi. Wakantanka hecon sdonyapi kin heon etanhan wakagiapi yuhapi kin hena ehpeyapi iyececapi. Tuka nakun iyowinyanpi sni kin heon Wakantanka sihda.

Unkan Israel iś eya ake Wakantanka iyokipiyapi śni. Koka kin he yulidokapi kte śni. - Wakantanka he tehinda. Koka kin mahen taku un he. Inyan wan akan woahope wikcemna owapi qon hee. Unkan Israel Koka qon yuhdokapi qa wanyakapi.

Oyate kin tohan Wakantanka anağoptanpi śni eca sihda ece. Hecen Israel iśs eya ake wowayazan wan tehika iyahdewicaye ça on nina țapi. Unkan Koka kin otonwe kin hen yuhapi kte cin ikopapi; qa otonwe tokanta wahowicayapi. Iś eya hena nakun Israel etanhanpi. Unkan huwe hipi; qa wicaśta wan paha akan ti; unkan he ti 
kin en ehnakapi. Hecen wicaśta qon he cinhintku kici Koka kin awanyakapi.

Unkan token on etanhan Śiloh ekta wośnapi wokeya wan he cin heciya Koka kin ayapi sni he. Ecin oyate kin he wakagiapi ohodapi, qa Wakantanka ohodapi sni kin he Wakantanka sihda; heon Koka tawa kin heciya ayewicakiye śni.

Wakantanka iśnana waonśida hinca; hetanhan Koka kin he ake Israel totonwepi kin ekta hdi, heon oyate kin tona Wakantanka waśtedakapi kin nina pidapi.

\section{WOONSPE 5.}

SAUL WICAŚTAYATAPI KIN.

Unkan tuwe Israel oyate en wicaśtayatapi he. Wakantanka Wicaśtayatapi tanka kin hee. Samuel wanna wicaśta tanka, qa wicaśtayatapi śni, tuka wayaco kin heca. He itokam Moses qa Josuwa qa Eli, hena Israel oyate en wayacopi kin heepi. Hehan iśs ito Samuel hee.

Samuel ohinniyan Wakantanka cekiye ça, oyate kin token econpi kta iyececa hecinhan he iwanga. Qa token eciya eca, ake is oyate kin owicakiyaka ece. Heon etanhan Israel pidapi kta iyececapi. Ecin wicaśta akantu wanji wicaśtayatapi yuhapi eśta Wakantanka he iyecece śni. Hecetu 
tuka wicaśtayatapi wanji wanyag yuhapi kta kecinpi; qa heyapi; Wicaśtayatapi wanji unhapi kinhan okicize eca he tokapa unkiyapi ece e kta, eyapi. Qa Samuel en ipi qa, Wicaśtayatapi wanji unqu po, eciyapi.

Samuel he on nina icanteśica, qa on Wakantanka cekiya. Unkan Wakantanka wicaśtayatapi wanji wicaqa he. Han. Wakantanka he iyokipi śni, tuka nina cinpi kin on, ito, wanji yuhapi kta ce, eya.

Hehan Samuel Israel oyate witaya wicakico qa hewicakiya; Wakantanka wicaśtayatapi wan niçupi kta ce. Tuka wicaśtayatapi kin he kaken niyuhapi kta ce ; nicincapi kin hena htani wicakiye kta ce; qa apa tacanpahmihma kin itokam yapi kta ce; qa apa iś mazasagye qa wahacanka wahonkeza ko kahwicakiye kta ce. Qa taku waśte yute kte cin hena iś nicunkśipi kin he śpankiyapi kta ce. Qa nitamagapi qa taku woyuha duhapi kin hena etanhanhan icu, qa iye hena yuhe ca, token cin kinhan ecen econ kta ce. Kinhan hehan wicaśtayatapi yacinpi kin de on iyopeniçiyapi kta ce: qa hehan hinnakaha Wakantanka ayaceyapi kta ce. Tuka iś hehan nanihonpi kte śni ce, ewicakiya.

Samuel hena hewicakiya, tuka nakun iyowinyanpi śni, qa: Wicaśtayatapi wanji unhapi kta, panyanhan eyapi, qa hehan owasin tiyata kihdapi. 
Unkan wicaśtayatapi kte cin he tuwe kahiniga he. Wakantanka iye kahnig̉e ça Samuel okiyaka, he tuwe kte cin.

Unkan kọska wan atkuku kin wanunyanpi suktanka śonśonna ko ota yuha. Únkan śonśonna kin etanhan yamni taninpi śni. Hecen kośka qon he ookiye wan kici śonśonna kin he owicadepi. Kośka kin he Saul eciyapi. Wanna tehan owicadepi. Tuka iyewicayapi śni; hecen unhanketa otonwe wan Samuel en ti qon he ikiyedan upi.

Unkan ookiye qon he Saul heciya; Otonwe kin den wicaśta wan waayata heca e ti: unkan taku eya eca, owasin iyecetu ece, keyapi nawahon ce. Ito, en unye ça śonśonna taninpi śni kin he unkiwange kte, eya. Hecen otonwe kin ehna yapi. Unkan Samuel akipapi. Tuka Saul qa ookiye kin unmanna tohinni Samuel wanyakapi śni, nakaeś tuwe kin sdonyapi śni. Samuel wanna wicalinca, onholida hanske cin heca wan un, qa paha kin hanskaska.

Unkan Saul heya; Waayate cin tukte ti hecinhan omakiyaka ye, eciya. Hehan Samuel is ayupte ça, Waayate cin hemaca ce, eya.

Unkan Samuel iye qe he tuwe kin sdonya he. Han, iye qe sdonya. Iśs eya tohinni wanyake śni, tuka Wakantanka he okiyaka, wicaśta wan hehan hen akipe kte cin he; kinhan he Israel en wicastayatapi kte cin hee kta. Qa Saul sonsonna kitan- 
in śni kin he iwanġe kta cin kin he nakun sdonkiya.

Hecen Saul okiyake śni itokam iye tokaheya heya; Śonśonna nicitaninpi śni qon wanna iyewicayapi ehantanhan anpetu yamni ce, eya. Hehan Samuel iye ti kin ekta hde ape. Qa hanyetu kin

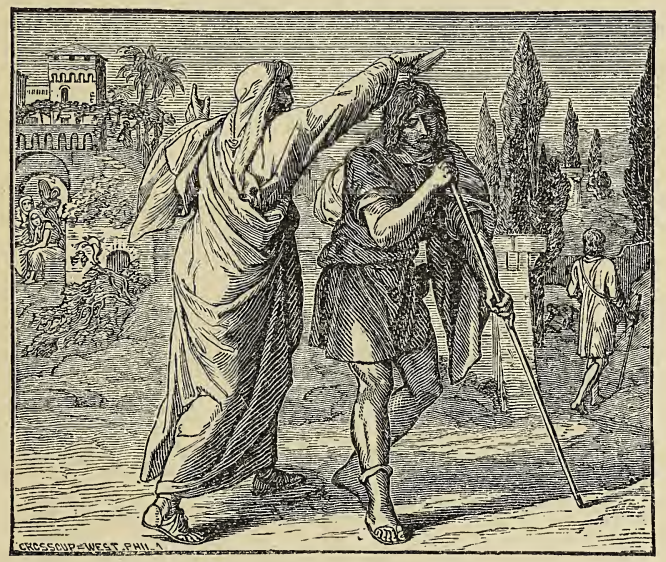

SAMUEL SAUL SDAKIYE.

hen kici yanke ça, taku ota okiyake ça, ihanhanna hehan hde kta keciya. Hecen Saul qa ookiye qon kici Samuel ti kin ekta hipi. Hehan Samuel Saul kiciśnana tipi kin akan ipi, qa heciya taku okiyaka.

Hehan ihanlianna qehan, hamlianna linea kiktapi, qa Samuel qa Saul, ookiye qon hena otonwe kin ehna yapi; qa ecen otonwe kin inaśdokapi 
qehan, Samuel Saul heciya; Ookiye kin he iśnana kahintokam hde śi wo, eya. Hecen ookiye kin iyaye çehan, hehan Samuel Saul kiciśnana inajin, qa wihdi janjan wan yuha i qon he icu, qa Saul pa kin akaśtan, qa heya; Wakantanka Israel en wicastayatapi kalinige cin he niye ce, eciya. He ehan tuwe wicaśtayatapi kta eca hecen econpi ece. Samuel hena hecakicon, qa hehan kinunkankiya kihdapi. Saul iye takuwicaye cin ekta ki, tuka wicaśtayatapi kte cin he tuwedan okiyake śni.

He iyohakam iecadan Samuel Israel witaya wicakico. Qa tuwe wicaśtayatapi kte cin he owicakiyaka. Qa hewicakiya; Wakantanka niyepi en wicaśtayatapi. Unkan yacinpi śni kin he sicaya ecanonpi ce. Heca eśta wicaśtayatapi wan yacinpi kin he duhapi kta ce; eye ça hehan Saul hee kin wicakipazo. Oyate kin Saul wanyakapi, unkan nina iyokipipi. Saul iśnana hanska, Israel en tuwedan iyehankeca śni. Israel oyate kin wicaśta hececa wanji he wicaśtayatapi yuhapi cinpi. Wicaśta hanske ca okicize en waditake cin he itanpi cinpi. Hehan oyate kin panyanhan heyapi; Wakantanka wicaśtayatapi kin de patan wo, eyapi.

Wicaśtayatapi kin he taku wicaśta kin nayahonpi kta; Wakantanka waśtedake ca iś hecece sni, unma tukte hecinhan. 
Unkan Israel tanyan econpi e on etanhan wicaśtayatapi waśte yuhapi kta kecannipi he. Hiya. Wakantanka cantewicakiye ça tanyan wicakuwa, tuka hee pidapi śni. Qa Samuel nakun wayaco yuhapi, qa taku owasin owicakiyaka ece, tuka hena e pidapi śni. Samuel heon canniye wicaye sni, tuka śicaya econpi kin he icansica, heon sanpa nina wahokonwicakiya.

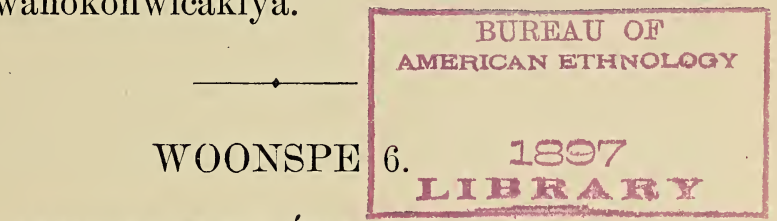

SAUL QA TAKU ŚICA ECON QON.

Wakantanka Saul token wicaśtayatapi kag̉e cin he wanna nayahonpi. Saul wateśdagkiton qa oyanke wan akan yanke ça, ozuye eca canpahmihma iyotan waśte kin heca ohna ya ece, qa okicize en waditaka.

Samuel ohinni Saul wanyag i, qa nina wahokonkiya; Wakantanka anaǵoptan qa wicaśtayatapi waśte kta he cinkiye, ça on nina wocekiye ekiciya.

Wakantanka Abraham waśte dake cinhan sdonye kta e on etanhan taku econ si. Unkan tokeh econ si qon iyecen econ, qa on etanhan Wakantanka eceedan wastedake ciqon he yeksuyapi. Iho he iyecen Wakantanka token cin kin hena Saul econ si kta; ito is token econ ka. 
Oyate wan Kanan makoce kin ikiyedan tonwanyanpi, oyate kin he Amalek eciyapi. Oyate kin he sicapi, heon etanhan owasin awihnunipi kta e Wakantanka wicakonza.

Anpetu wan en Samuel Saul yanke cin en i, qa heciya; Amalek oyate kin he wicayakize kta ce. Wakantanka he econ niśi ce; hecen wica, qa winolinca, qa śiceca, qa wanunyanpi taku yuhapi koya iyuhpa wicayaktepi kta ce, eciya.

Hecen Saul akicita ota iwicacu qa oyate kin he takpe wicai, qa wicaktedan, qa Wakantanka hecon si qon econ, qa mazasagye on owasin wicaktepi. Unkan Saul hecin; ito wicaśtayatapi kin he niyake awahda ke ecin; qa heceedan ktepi śni tiyata aki. Saul iye wicaśtayatapi iyotan, qa on etanhan wicaśtayatapi wan kaśka hnake ça on iwahaniçida kta kecin. Qa nakun tahinca qa wanunyanpi tona tamaheca ślitinpi kin henana wicakte, qa tona cepapi waśtepi kin hena niyake awicahdi. Hena on wijiniçiya cin qa hecon.

Unkan hanyetu wan en Wakantanka Samuel okíya, qa Saul olian kin nina iyokipi śni he okiyake. Samuel he nahon kin nina iyokiśica, qa hanyetu ihunniyan Wakantanka cekiya.

Thanhanna qehan Samuel Saul ode, qa Wakantanka taku ota eciye ciqon hena Saul okiyag śi. Saul sicaya econ kin nahahin Samuel sdonye śni kecin, qa on hnaye kicaga. 
Hehan Samuel wanyake çehan iyuśkin, tuka he ecaheconka. Qa heya, Wakantanka token econ maśi qon ecen ecamon ce, eya. Tuka Samuel iś heya; Qa tahinca qa wanunyanpi ko hoton yukan nawation kin dena takupi he, eya.

Wanna Samuel hena sdonye cin Saul sdonkiya, hecen takudan ni wahnake śni ce, eye kta iyecece śni nakaeś, wancahna ihdatokan qa oyate kin he iye econpi ce, eya. Unkan Saul he iye sni, tuka oyate kin hee heconpi he. Hiya; iye itancan, tuka hecen cin nakaeś, oyate kin iyecen econpi.

Unkan wanunyanpi kin hena Wakantanka wakiyuśnapi kinhan iyokipi naceca he. Hiya. Saul iye Wakantanka anagoptan kinhan he iyokipi kta tuka.

Hehan Samuel Saul heciya; Wakantanka canniyeniyan ce, hecen wicaśtaniyatapi kin de tehan kte śni ce, eya.

Saul he nakilion kin nina yuśinyaja. Qa Samuel heciya; Miciyanke ça wocekiye emiciya ye. Saul wahtani kin on iyokiśice śni, tuka Wakantanka kakiśsye kta ikope cin heceedan on hecece cin, Samuel he sdonya. Wakantanka Saul tanyan ecakicon, unkan wahtani. Tuka nakun cante kin on iyopeiçiye śni ; tuka wikopa ecedan on hecece cin. Heon Samuel kici yanka cin śni. Qa Samuel hde kta, unkan anica, qa taonhohida kin en 
yuza; tuka Samuel iyowinye śni kihde ça taonhohia qon kiyulideca.

Unkan Samuel kinajin qa heya; Wicaśtaniyatapi tuka Wakantanka he enicihdaku, qa wicaśta wan niye kin isanpa waśte e he qu ce; qa wanna he yuśtan ce; hecen Wakantanka tawacin hdutokeca kte śni ce.

Hehan iyotan nina yanke ape, qa kici Wakantanka cekiye kta keya. Wakantanka canniyeye cin he oyate kin sdonyapi kta cin śni. Saul Wakantanka iyotan dake śni, qa oyate e iyotan wicada, unhanketa Samuel kici cekiye kta keya.

Hehan Samuel Amalek wicaśtayatapi wan niyake yanke cin he wanyaka cin. Wicaśta kin he sica, tawacin hinyanza, heon etanhan ktepi kte cin he Wakantanka konza. Tuka Saul ni hnake cin heon iye kin, wani kte sece ecin. Tuka Samuel hehan wanyaka, unkan mazasagye wan icu qa kte.

Hehan Samuel Saul elipeya kihda, qa icimana wanyag hi śni. Tuka Samuel ohinni iyokiśica, Saul śicaya econ kin heon.

Iho, micinca, Wakantanka iyokipi śni yayapi kinhan, nicantepi kin on iyokiśica po. Saul Wakantanka waśtedake śni, tuka kakiśye kta ikope cin heceedan ekta ewacin.

He tokeca Saul wicaśtayatapi kin kte śni he, qa wanunyanpi kin hena nakun. Ecin Wakan- 
tanka hena owasin awihnuniye sii, tuka oie kin anagoptan śni kin he śicaya econ. Hecen unkiśs eya Wakantanka taku econ unśipi kin hena econqonpi kte. Unkan Wakantanka tona śicapi kin hena wicakte unśipi he. Hiya. Owasin cantekiciye unśipi.

\section{WOONSPE 7 .}

DAWID TALIINCA SKA AWANYAKE CIN.

Hehan tuwe wicaśtayatapi kte cin Samuel nahanliin sdonye śni. Unkan Wakantanka heciya; Ihepi ojuha kin he ojudan ohdaśtan, qa Bethlehem ekta ya wo; wicaśta wan Jesse eciyapi hen ti kin he cinca kin wanji wicaśtayatapi wakaliniga ce.

Hecen Samuel Bethlehem ekta Jesse ti kin en i. Jesse wanna wicahinca, qa cinca kośka ota. Unkan Samuel Jesse cinca tokapa kin he wanyaka, unkan ito eca Wakantanka kahnigge cin he dee ka ecin. Kośka kin hanske ça wicaśta waśte, qa wicaśtayatapi oiyokipi kta iyececa. Unkan Wakantanka kahigige cin he dee ce, Samuel ecin.

Tuka Wakantanka Samuel heciya; Wanji wakahnig̉e cin he dee śni ce. Wakantanka wicaśta itohnake iwanyake śni, tuka wicacante kin hee iwanyaka. Jesse cinca tokapa kin owanyag waśte, tuka cante kin e Wakantanka iyokipiye śni. Han. 
tuqe tuwe cante kin on Wakantanka waśtedake cinhan he Wakantanka wicaśtayatapi kahinige kta.

Hehan ake Jesse cinca okihe kin he wanyaka; tuka, Wakantanka kahinige cin dee śni ce, eya. Qa ake wiciyamni kin he wanyaka. Tuka nakun

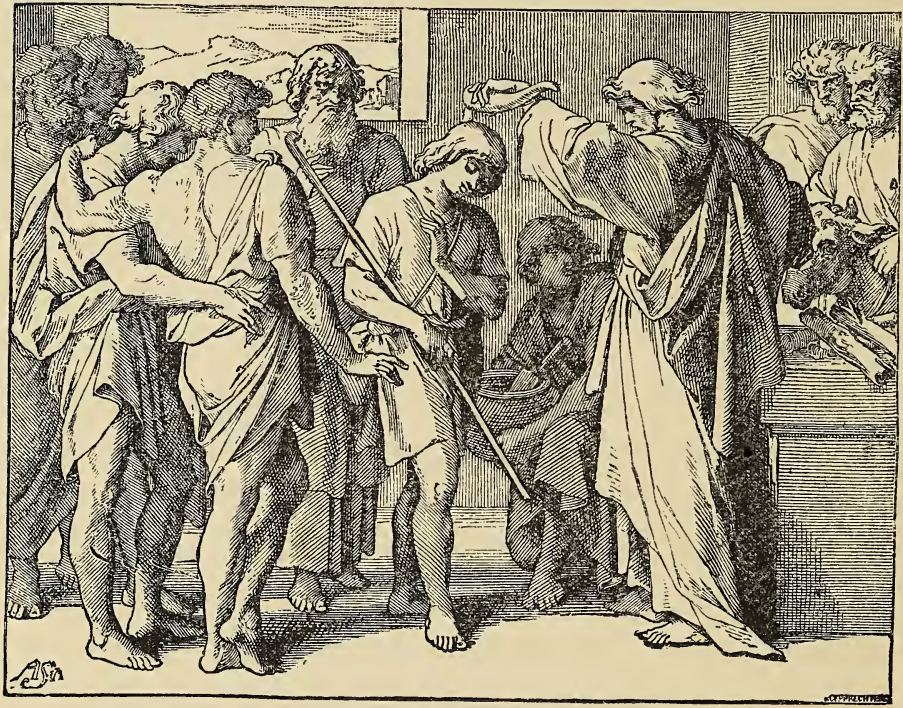

SAMUEL DAWID SDAKIYE.

hee śni, ecen cinca kośka śakowinpi kin owasin wanwicayaka. Tuka wanjidan Wakantanka kahinig̉e cin eepi śni.

Unkan Samuel Jesse heciya; Nakun nicinca wanji tokiya un he. Unkan Jesse ayupte ca, Ehake micinca wanji, tuka tahinca wanunyanpi 
awanyaka ce, eya. Hehan Samuel, He kohanna kico ye wicaśi wo; wanmdaka wacin ce, eya. Qa Jesse kico ye wicaśi.

Jesse cinca hakakta Dawid eciyapi. Nahanhin kośka śni hokśidan. Dawid ite waśte kin ecen iśta oze koya tawacin waśte itohnake. Unkan cante kin on Wakantanka waśtedaka he. Han, waśtedake.

Unkan wanna en hi, hehan Wakantanka Samuel heciya; Najin qa ihepi kin on ecakicon wo, de wakahinige ciqon ee ce. Hehan Samuel ihepi kin on pa sdakiya; cincu śakowinpi kin icahda najinpi.

Hehan Samuel iye ti kin ekta kihda. Dawid wanna wancahna wicaśtayatapi kta Wakantanka ke śni. Tuka Dawid iś eya sdonkiya, tokata anpetu wan en wicaśtayatapi kte cin he. Hecen ake iś tahinca wanunyanpi awanyaka. He awanwicayake cin icunhan candowankiyapi on dowan. Wakantanka yatan dowan. Dawid tadowan kin hena Psalm eyapi.

Dawid wicastayatapi cin hinca kecanni he. Wicaśtayatapi oyanke qa wateśdake kin hena cin kin ee śni. Taku iyotan da kin he oyate kin witaya Wakantanka yatanpi kta, hee cin kin ee. Iye itancan kinhan he onspewicakiya okihi; qa tona onśikapi kin hena onśiwicada okihi; qa tona iś śicapi kinhan, nakun kakiświcaya okihi. 
Samuel Dawid ihepi on pa sdakiye ciqon he icunhan Wakantanka Taniya Wakan kin Dawid qu; hecen nina ksape ça waditaka, qa wicaśtayatapi kta iyececa. Wakantanka iśnana wicoksape qa wowaditake kağa okihi.

\section{WOONSPE 8.}

DAWID QA CANDOWANKIYAPI KIN.

Unkan tuwe wicaśtayatapi kte cin Saul sdonye śni. Ecin Samuel okiyake śni. Dawid hee kte cin Saul sdonye cinhan nina sihda kta, tuka sdonye śni.

Saul ohinni cante śica. Woniya sica cante kin iyohpaya. Tuka Wakantanka anakicipte śni. Hecen nina wikope ça icomni un kin hena asni śni. Tipi iyotan waśte kin heca oti, qa taokiye kin iś ota hena yuonihanyan kuwapi, tuka takudan icanwaśte śni.

Tho micinca, he sdonya po. Wakantanka tohan taku śihda eca, iye iśnana iyokiśinwicaya okihi, qa nakun iyuśkinwicaya okihi.

Saul cante sice cin heon token econ he. Wahtani kin heon iyopeiçiye ça Wakantanka kicicajuju kta icekiya he. Hiya. Wocekiye eye kte cin waśtedake śni.

Hehan Saul taokiye kin sdonyapi, woniya śica 
cante kin mahen un, qa on etanhan hecece cin he, qa heciyapi; Okinni candowankiyapi qa wicaho ko on dowanpi nayahon kinhan anisni kta nace. Hecen yacin kinhan tuwe wayupika wanji unkodepi kte, eyapi.

Hecen Saul awicayupte ça wanji ode wicaśi. Unkan ookiye qon wanji Saul heciya ; Jesse Bethlehem ekta qon he cinca kin wanji heca wayupika sdonwaya ce; qa owanyag waśte ie ksapa ko; qa nakun Wakantanka waśtedaka ce; he kico yapi yacin he, eya.

Unkan Saul he kico ye wicaśi. Unkan Jesse ekta ipi qa heciyapi; Wicaśtayatapi kin he unkuśipi, qa Dawid nicinkśi kin he kico ce, eyapi.

Unkan Jesse iś Dawid ye kta keya. Hehan tatokadan tado qa ağuyapi miniśa ko wicaśtayatapi kin kaye śi. Unkan Dawid iś śonśonna wan hena qinkiye ca ekta kai. Hehan Saul Dawid wanyake cin nina waśte dake ca, tehan en un kta cin; qa Dawid atkuku kin ekta okiyag wicaśi. Dawid nina candowankiye cin Saul nahon yanke cin heon tawacin kin ekicetu aye ça ecen akisni.

Hehan ake Dawid Saul ehpeya kihda, qa ake tahinca wanunyanpi awanwicayaka. Dawid wicaśtayatapi ti en un kin he iyotandake śni, tuka tahinca wanunyanpi awanyake cin hee iyokipi. Saul wicaśta śice nakaeś, taokiye kin ota iś eya sicapi. 


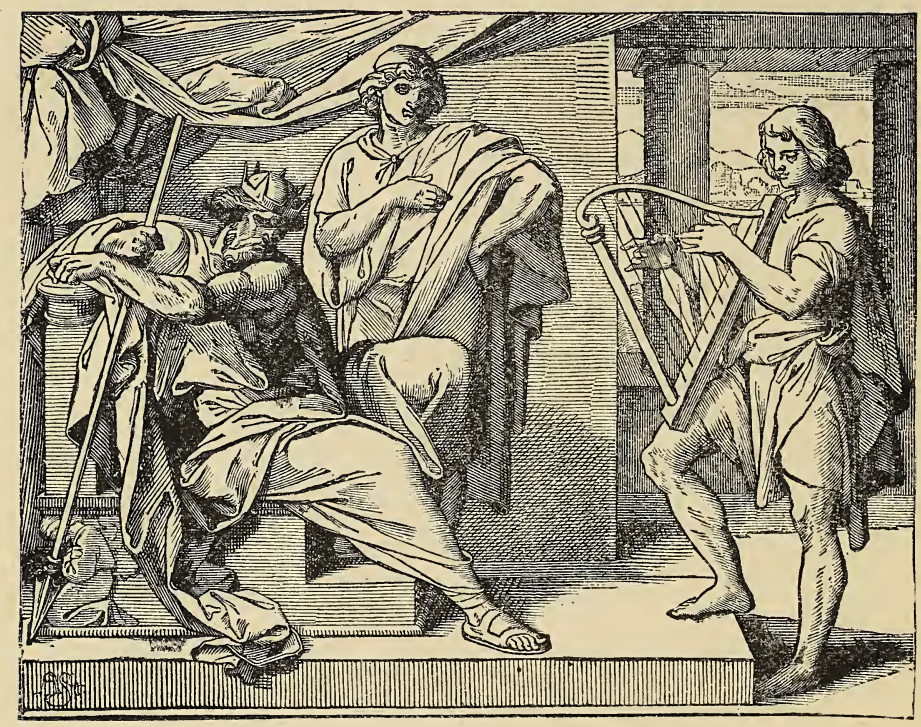

DAWID SAUL ITOKAM CANDOWANKIYE.

Dawid tohan iśnana un eca nina iyuśkiniçiya ece. Hehan Wakantanka nina awacin un kin heon hececa. Dawid tadowan kin wanji en kaken eya; Jehowa Waawanhdake mitawa kin hee; Heon iwicakakije wanin waun kta ce.

Dawid tahinca wanunyanpi tanyan awanwicahdaka. Tukten wato śtunka waśte kin he en awicaya; qa mini amdakedan mdezedan kin hen awicaya. Qa wakpadan kahdaya tukten wato panpanna cusni kin he en iwankewicaya. Dawid tahinca wanunyanpi kin tanyan wicakuwa kin he 
icunhan Wakantanka iś iye isanpa Dawid tanyan awanyake cin he awakicin qa heya; Peji toto wanke cin en iwangmaye ça, mini amdakedan icahda amayan ce.

Hokśipidan, wicinyanpidan, tokin niś Itancan Waawanhdake duhapin. Jesus Waawanhdake waśte kin hee. Waśtenidakapi on iye wiconi ehpekiye. Hecen tona wacinyanpi eca cantewicakiye ça, iyokiśicapi icunhan iye tokicanpte kin on iyuśkinwicaya.

Hokśiyopa tona Jesus waśtedakapi kin hena nina iyuśkinpi.

\section{WOONSPE 9.}

DAWID QA GOLIA WICAŚ'TA TANKA QON.

Oyate wan śicapi e Pilistim ewicakiyapi, Kanan makoce kin en tonwanyanpi; unkan he Israel nina tokawicayapi nayahonpi qon.

Ake anpetu wan en Pilistim wicaśta au qa paha wan akan ahitipi. Saul he nahon, unkan iś eya Israel wicota om ye ca, iś icunonpa paha wan akan etipi. Hecen anokatanhan wankiciyag tipi, qa kicizapi kta e napin piic̣iyapi, tuka nahanhin econpi śni.

Wicaśta wan Golia eciyapi, Pilistim oyate kin 
etanhan ; wicaśta tanka qa hanska. Siha iyutapi wikcemna hehankeca; waśake ça kicis wohitika, qa kicis ihduza. He ehan oyate kin maza wokoyake kaġapi, qa okicize eca he unpi, hecen wanhinkpe qa mazasagye kin hena nina kiunniwicaye śni.

Unkan heca koyaka. Taonliolida kin maza, qa tawapaha kin iś mazazi, qa huha qa siha ko aiyahdeya mazazi kin heca koyaka. Qa ite kin ecedan śdayehna un tuka nakun maza wahacanka wan yuha. Hecen ite taopi kta eśta he inakipe kta. Qa mazasagye wahonkeza ko yuha.

Golia hanske cin, tuwedan makte kta okihi śni, ecin. Anpetu eca ake Israel wicakipan, Tuwe makiza okihi hecinhan kici ecamon kte, eya han ece. Qa nakun heya; Tuwe makte okihi qa makte kinhan, hehan token yacinpi kin Pilistim iyecen econpi kta ce. Qa miś he tuwe kin wakte kinhan, unkiś token uncinpi kin ecen ecanonpi kta ce, Israel ewicakiya.

Golia heyahe cin Saul taoyate om nation kin nina inihanpi. Tuwe he kiza eśta itkom kte ehantanhan, unkiś nakun owasin unktepi kte ecinpi. Israel Wakantanka wacinyanpi kinhan ówicakiye kta tuka. Dawid cincu yamni hen opapi, tuka Dawid iś he icunhan tahinca wanunyanpi awanyaka.

Unkan Jesse Dawid heciya; Agruyapi wikce- 
mna qa wamnaheza onge iyahna icu, qa nicinye wicakaya wo ; qa ito eca token unpi ka, eya.

Hecen Dawid hanhanna hin kikta, qa ağuyapi wamnaheza ko yuhe ça iyaya. Israel paha wan en tipi kin ekta $i$, qa cincu owicakide, qa iyewicakiye ca om wohdaka han, he icunhan tuwe panyanhan heya; Tuwe makiza okihi hecinhan kici ecamon kta, eye. Golia heya ece kin Dawid tohinni nahon śni; unkan icahda najinpi kin hena Dawid heciyapi; Tuwe Golia kte kinhan taku ota on kicicajujupi kta, Saul keya ce, eyapi.

Oyate kin Golia kokipapi kin he Dawid iyoyakeca. Ecin Wakantanka Israel ówicakiye cinhan ohiyapi kta tuka kin heon. Qa iye Pilistim taku wakan yuhapi kin he ówicakiya okihi śni. Ecin he wakag̉api, hecen takudan okihi śni. Unkan Dawid heya; Pilistim kin de tuwe ca, Wakantanka taoyate kin decen tamwicakiya he. Qa Dawid cante mahen Golia kize kte cin kokipe śni.

Dawid heye cin cincu kin nahonpi; unkan on etanhan wanji unce ca, Tahinca wanunyanpi kin ekta awanwicadake śni; den e takilian yahi he, eciya. Tuka Dawid iye qe itkom cincu kin ihnahna ayupta.

He icunhan Saul okiyakapi, Dawid Golia kize kta tawațenye cin he. Unkan Saul Dawid wanyaka cin; hecen Dawid Saul en i. Saul he itokam Dawid wanyake ciqon akiktonja. Saul Dawid 
wanyaka, tuka ecedake śni, qa heya; Nahanhin honikśidan qa tohinni kicizapi ecanon śni; hecen Golia yakize kta oyakihi śni, eciya.

Tuka Dawid iś ayupte ça heya; Eya ate tahinca wanunyanpi tawa awanwicamdaka, unkan mnaja wan en hi, qa tahinca wanunyanpi optaye kin etanhan cinca wan akiyahda; tuka etawape ça wehdeġa, qa mnaja i kin etanhan tahinca wanunyanpi kin ewehdaku. Unkan mnaja kin makte kta, tuka hin kin kagge mduze ça ecen wakte çe. Qa nakun eya wahanksica wan en hi, tuka nakun wakte. Hecen mnaja wan wahanksica wan iyahna wakte ce. Tuka he miye cinka śni. Wakantanka he iye, mnaja qa wahanksica śakepi kin etanhan nimayan ce; hecen ake dehan Pilistim wicaśta kin de etanhan nimaye kta ce; eya.

Dawid iye ohitika on wowitanye śni. Tuka Wakantanka he ókiye cin sdonkiya. Saul hena nalion unkan hehan Dawid Golia kize śi. Tuka Dawid mazasagye qa maza wokoyake on ikicize ko nica; hecen Saul iye tawipe qa iye tawokoyake ko Dawid unkiya. Tuka, Tohinni deca mun śni ce, eye ça, owasin yuśdoka, qa mazasagye qa wahonkeza ko takudan icu śni. Tuka wakpadan wan ekta i, qa inyan zaptan śduśduta kahinige ça wojuha wan yuhe cin en okihnaka. Qa inyan yuhmunpi wan yuhe ça, cansakadan sagye wan yuhe ciqon he iśs napsanni hduhe ça Golia takpe ya. 
Golia he nakilion, Israel etanhan wanji kis u kte cin he: qa iś eya itkokim $u$; qa wanji tokeca tawahacanka kin itokam kicicaya. Unkan Grolia Dawid wanyake cin, tokeś wanji iye iyececa hanska tanka, qa wipe tonyan u wanyake kta kecin.

Tuka Dawid nahanhin hokśidan qa itohnake koya nahanhin hokśiyopa itohnake: qa tanina to-

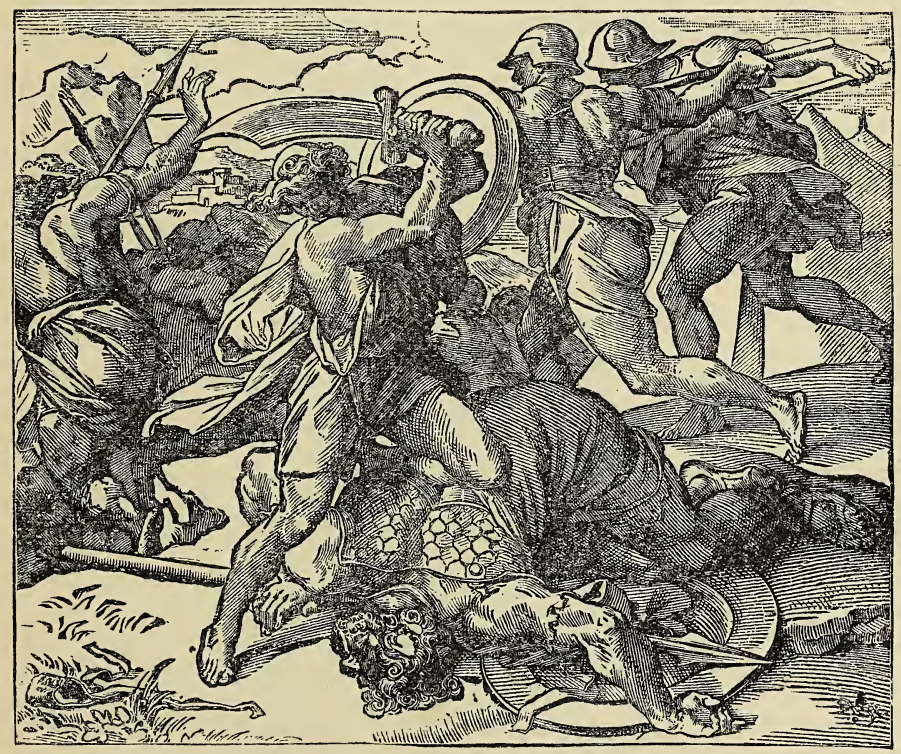

DAWID GOLIA KTE.

ken ihdus un qon hecehnana; qa cansakadan sagye wanjidan yuha.

Golia Dawid wanyag iheya, unkan sanpa śi- 
hda, qa yaśice ça, ikicapa, qa akeś hankeya ihalia qa heya; Mikiyedan u wo, nitancan kin he zitkadan qa wamanica ko niyutapi kta e wicawaqu kta ce, cciya.

Tuka Dawid iś ecaca kokipe śni, qa iś eya heciya; Niye mazasagye wahonkeza wahacanka ko duha ce; tuka miś Wakantanka he nicismakiye kta, qa iye omakiye kta; hecen cikte qa nipa kin he iwacu kta ce. Qa Pilistim nakun wicaktepi kta, qa cehpipi kin owasin wamanica zitkadan ko temwicayapi kta ce. Kinhan hehan Israel Tawakantanka kin heceedan Wakantanka kin he oyate sdonyapi kta, qa iye tuwe cin eca nikiya ece, cya.

Hehan Golia Dawid ikiyedan ya. Tuka Dawid iś inyan qeya yuhe ciqon wanji icu, qa inyan iyuhmun kin en okihnake ça, hehan etkiya awicakehan naśdog iyaye ça, token waśaka hecen inyan iyuhmun on kinin, qa ite kin cokaya kaohpa ape ca ecen kaohdapśinyan hdilipaya.

Hehan Dawid anatan, qa tamazasagye kin icu, qa pa kin kaksa ehpeya. Wakantanka woyuśinyaye wan tanka econ kin Pilistim he wanyakapi kin on nina inihanpi.

Hehan Israel iś Wakantanka he ówicakiya e wanhdakapi; qa nina iyakiśapi; qa hehan Pilistim kin anawicatanpi. Unkan Pilistim oyate kin nakipapi, tuka wicakihdegapi qa ota ewicaktepi. 
Hehan Dawid iś Grolia tawokoyake kin hena yuhe ça, pa kin nakun Israel wicakipazo.

Hehan Dawid Wakantanka wopida eciya, qa candowankiyapi on yatan dowan. Dawid iye atayedan oyate yatanpi cin śni; tuka wicaśta otoiyohi Wakantanka e yatanpi cin; qa oyate kin decen eyapi cin: Wakantanka ecedan tanka ce. Hokśin wahipanica wan tahinca wanunyanpi awanyake ciqon okiye ça, Golia wicaśta hanske ciqon ktekiya ce, eyapi cin.

Oyate kin tona witantanpi kin hena iye wicayatanpi ecedan cinpi; tuka tona Wakantanka waśtedapi kin hena Wakantanka e oyate owasin yatanpi cinpi.

Tho he iyecen, micinca, Wakantanka onśinidapi, qa taku waśte econniyanpi kinhan, heon iye yatan po; qa niye niwaśtepi on ihdatanpi śni po. Itancan tawocekiye kin en, Nicaje kin wakandapi kte, eye ciqon he hecen ka; oyate owasin yatanpi cin.

\section{WOONSPE 10 .}

DAWID QA WAHONKEZA KIN.

Hehan Saul nina iyuśkin, Dawid Golia kte kin heon; qa Dawid Saul en aipi. Dawid Golia pa qon yuha Saul yanke cin en i. Unkan Saul heya; Kośka tuwe ateyaya he, eciya. Dawid 
ayupte ca, Jesse Bethlehem en ti kin he atewaya ce, eya.

Saul Dawid kici wohdake cin he icunhan Saul cinhintku Jonatan, he wicicahda najin; wicaśta waśte icağa, qa nakun waditaka.

Jonatan Dawid wanyake cin wancahna waśtedaka. Taku on Jonatan Dawid waśtedaka he. Ecin Dawid owanyag waśte qa nakun waditake cin heon; qa kokta Wakantanka Jonatan Dawid waśte dakekiya. Hecen waśtedake linca; qa ohinniyan napin cantekiciyapi kta kekiciyapi. Jonatan tawokoyake qa tamazasagye ko Dawid qu.

Hehan Saul, Dawid iye atkuku ti kin ekta hde kte śni, tuka iye ti kin hen ohinni un kta, keciya. Hecen Dawid Jonatan kici ohinni wankiciyag unpi, kodakiciyapi napin unpi kin heon ohinni ikiciyuśkinpi.

Okicize kin iyohakam Israel oyate iye totonwepi kin ekta aki. Hehan Saul qa Dawid qa Jonatan hena wicaśtayatapi tipi kin en hlapi. Unkan winolinca wicota candowankiyapi on dowanpi qa wacipi wanyag hdapi. Dawid e ota kte qa Saul e aoptetu eya dowanpi. Qa wocajeyate kin kaken eyapi; Saul kektopawinge wanji kte, qa Dawid e kektopawinge wikcemna kte ce, eyapi.

Unkan Dawid odowan kin de nalion kin iyokipi kecanni he. Hiya; Dawid iye yatanpi cin śni; tuka Wakantanka e yatanpi cin. Qa wino- 
hinca kin hena kaken eyapi kinhan hee nalion cin ; Tahinca wanunyanpi awanyaka onśike ciqon Wakantanka okiye ça Golia ktekiya ce, eyapi cin.

Tuka Saul iś odowan kin de nakilion kin nina sihda. Iye yatanpi cin. Tuka Dawid e iye kin isanpa yatanpi kin he ecaca nahon cin śni. Qa hecin, Takeyapi se; Dawid iye wakte kin isanpa kte keyapi se; qa wadimatake cin Dawid iye misanpa keyapi se; qa heon Saul cante sicaya yuza. He inakiwizi kin heon. Joseph cinyewicaye cin iyewicaceca qa Qain iyececa. Hehan Saul Samuel heciye ciqon he kiksuya: Wakantanka niye kin isanpa wicaśta wan waśte kahinigge ẹa hee wicaśtayatapi kta ce, eye ciqon he ehanqon Dawid de hee kta hunśte, ecin.

Saul hecin kin hee kta he. Han, hee kta. Hetanhan Saul Dawid sicedaka qa kte kta cin. Qa Saul iye ti kin ohna un kin ohinni cante sicaya yus un. Wakantanka onśiwicada qa owicakiye ca, Israel ohiyapi he pida kte cin hee, tuka piyedeśin Dawid token țeye kte cin he awacin un.

Hecen iye ti kin en hdi kin iyohakam, ake woniya sica Saul cante kin iyolipaya; tuka Wakantanka anakicipte śni. Unkan Dawid iś token han he. Candowankiyapi ehdaku qa kidowan ece qon ake econ, qa odowan waśte ahiyaya. Tuka wanna Saul wahonkeza ptecedan wan pe icu qa, 
Ito de on Dawid icinin cawapa ke ecin, qa hecen on kinin. Tuka Dawid wanhdake ça napa, hecen paśna. Tuka ake nakun icu qa on kinin, tuka

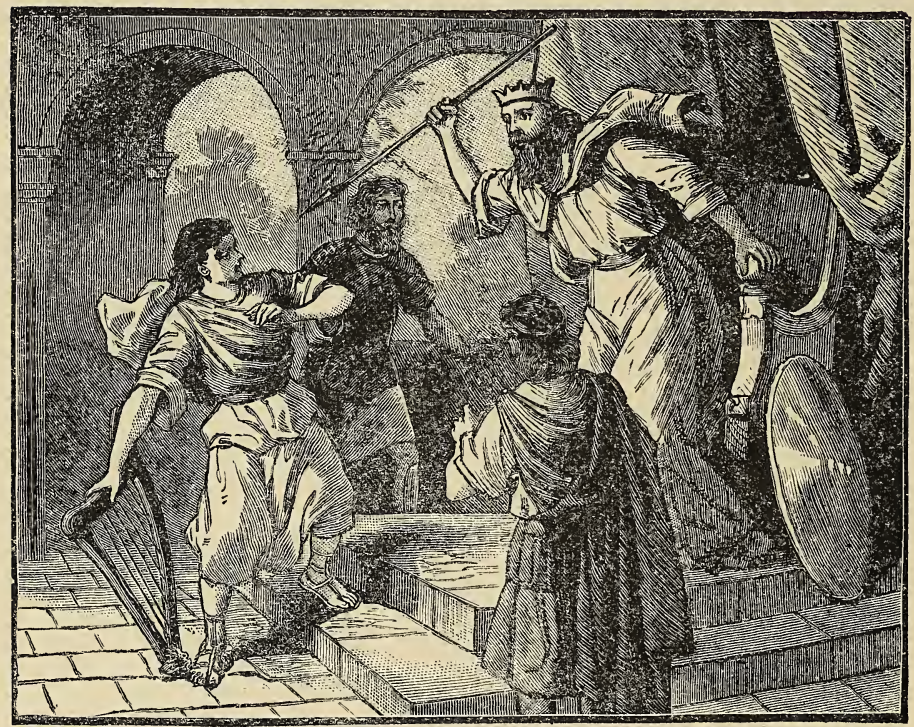

SAUL WAHONKEZA ON DAWID ICININ.

nakun kiunniye śni. Ecin Wakantanka Dawid awanyake cin heon.

Owasin Dawid e waśtedakapi, heon sanpa Saul Dawid sicedaka. Tuka Dawid iś ohinni tanyan econ. Hecen Saul Dawid taku śica wanji iyaonpe ça on kakiśye kta kecin, tuka takudan iyaonpe kta iyeye śni. Unkan Sanl Dawid akicita om ake Pilistim wicakis yeśi. He tokeca Saul ake Dawid 
wicakis yeśi he. Okinni okicize kin en ktepi kta ecin, qa heon yeśi. Tuka Dawid ake Pilistim ota wicakte, tuka iye kiunniyanpi śni. Heon oyate kin sanpa Dawid yatanpi, qa yawaditakapi. Heon Saul iś sanpa ślhda, qa unhanketa taokiye kin owasin Dawid kte wicaśi, Tuka ookiye kin owasin Dawid waśtedakapi; heon ktepi cinpi śni. Hehan Jonatan Dawid ktepi kta ikopa qa inahbe si ; qa iye atkuku Saul cekiye ça Dawid niye si kta keya. Qa Jonatan atkutku heciya; He tokeca Dawid yakte yacin he; taku tokanicon he; Golia kte qa iyuśkinniye sece ciqon, taku on nakaha canniyeyaya he, eciya.

Jonatan Dawid cantekiye ça ni cinkiye linca, hecen Saul Dawid kte kte śni keya wahoya. Hehan Jonatan ake Dawid kico qa Saul en ai, qa ake om un.

Tuka ake ecahankeya Saul Dawid sicedaka. Ecin nahanliin woniya śica cante kin mahen un. Dawid iś ake candowankiyapi on dowan, qa iyokipiya cin. Tuka ake Saul wahonkeza qon hee on icinin. Tuka Dawid napa; unkan tipi kin ee ha capa.

Hehan Dawid en un kte cin kokipa, qa haniyotahedan najica. Tuka Dawid atkuku ti kin ekta hde śni. Ecin Saul heciya ode kta, heon icunonpa naic̣ilima un. Tuka Wakantanka Dawid waśtedaka, hecen awanyaka. Tuka Saul iś wakanśica 
iyecen winawizi qa witantan, heon nina canteśiniçiya.

Micinca, tokin niś otoiyohi waśteyecidapin, qa niyepi kin wanji niyatanpi eśta inakiciwizipi śni po; tuka hee on sanpa ikiciyuśkin po.

\section{WOONSPE 11.}

DAWID QA IMNIJA OḦDOKA KIN.

Dawid Saul etanhan nape kte çehan, takodaku Jonatan elipeya ye kta tuka; Jonatan iś atkuku kici un kta ic̣ida. Hecen kinunkankiya yapi kte çehan, iikiciputakapi qa ceyapi. Tuka Jonatan iś owanji un kte cin he hecetu. Hehan Dawid iś Kanan makoce en hośki paha tankinkinyan qa imnija ohidoka ohna naicilima.

Hośki kin he ehna oyate tonana ounyanpi ; qa he ehna wato śtunka owihan waśte, hena ecen talinca wanunyanpi tanyan wilianpi; qa tatokadan kin iśs paha kin hena ośkatapi.

Dawid iśnana un śni; tuka cinyewicaye cin ekta hipi, qa om un. Qa hen oyate unpi kin onśikapi, tuka Dawid cantekiyapi qa okiyapi. Ecin Saul taoyate kin Dawid kte wicaśi. Tuka oyate tonana kin de iś Dawid tawaśiyapi; qa iyotan Dawid Wakantanka tawaśitku kin hee.

Dawid tawaśitku om witaya imnija ohdoka 


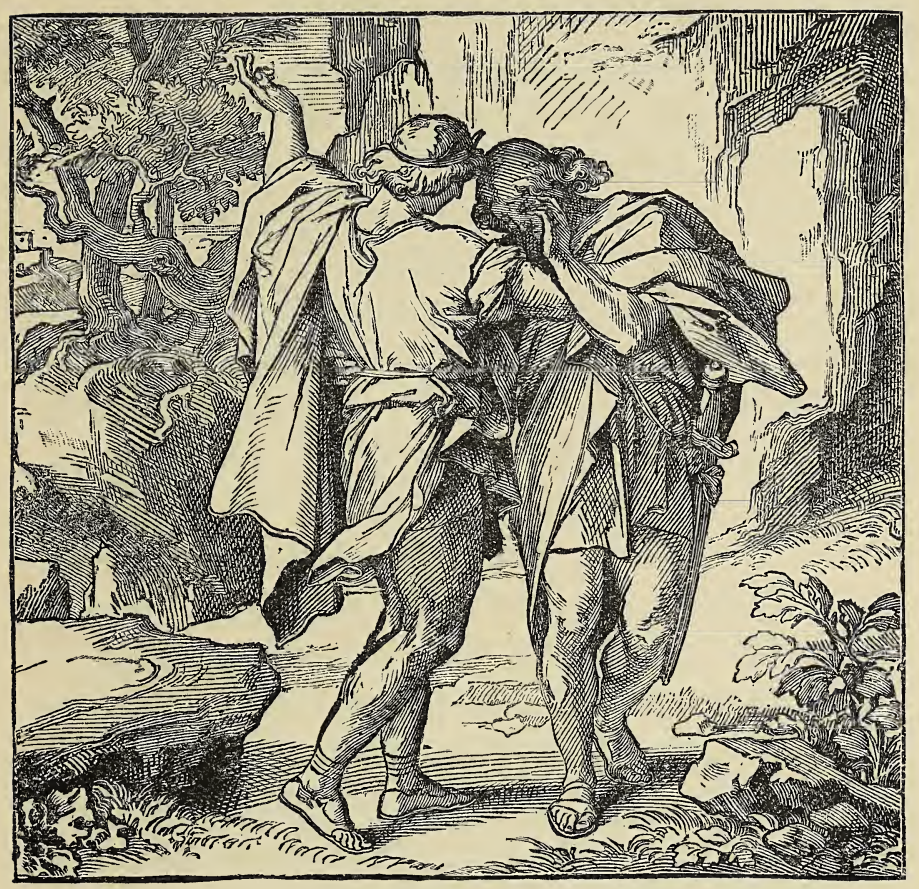

DAWID QA JONATAN.

wan otipi. Ohdoka kin wanjikji tankinyanyan, hecen wicokcota wicakipi ece.

Dawid tawaśitku om ohdoka mahen tipi, hecen tokiyatanhan taku yutapi tonpi he. Wakantanka wicaśta wanjikji Dawid waśtedakapi wicakağa; qa hena eepi wowicakayapi; qa neciyatanhan taku yutapi tonpi. Saul hena nahon, unkan heon sanpa cante okiçunnica, qa oyate kin hewicakiya; 
Dawid he wicaśta śica ce, makte cin ce, eya. Unkan Sanl taku eye cin heon wanjikji wicadapi, qa he hecetu kecinpi; qa hehan Dawid wanyakapi unkan ihaliapi qa śikśicaya ecakiconpi.

Saul oitonśni woyake ça he wanjikji wicadapi kin heon Dawid iyokiśica. Tuka Wakantanka he wokicanpte qu; qa tuktekten Dawid Wakantanka cekiye ça cante kin wanyake śi, Saul kte cin keciyapi kin heon. Tuka hecen cante yuze śni kin he Wakantanka sdonya. Dawid candowankiyapi hduha un, qa ohinni Psalm odowan hdohiyaya, qa oyate ktepi kta e iawanyag kuwapi, tuka Wakantanka he awanyaka sdonkiya, qa on nina yatan dowan.

Dawid imnija olidoka otakiya oti un, qa tuktekten contanka ehna naiçilima ece. Ecin wanjidankiya yanka eśta okinni Saul ode kinhan iyeye kta ikopa heon.

Saul taakicita ota om Dawid ode, tuka iyeye kte śni Wakantanka ecin kin heon iyeye śni. Unhanketa Saul tuwe wanyaka eca ake heciya ece; Dawid tukten un hecinhan omakiyaka po, he makte cin kin heon etanhan owade ce, eya. Eca okiyakapi, qa tuktetu kin en i keś, Dawid iś eya wanna ode kin nakilion, hecen ake tokan iyaya ece; hecen iyeye śni.

Imnija olidoka kin hena mahentu kin oiyokpaze hinca. Unkan ake heca wanji ohna Dawid 
tawaśitku om mahen iyotanka. Unkan he ican Saul iś taakicita om ohdoka kin he iyayuptapi. Tuka Dawid he mahen un kin sdonye sni; qa Saul wanna hehanyan mdokița, unkan taakicita kin olidoka kin itankan najin wicaśi, qa iye iśnana tin ye ça iśtinbe kta keya. Hecen tin i, tuka oiyokpaze nakaeśs takudan wanyake śni, tuka iye qe timahen yukanpi kin wanyakapi. Dawid Saul wanyaka unkan iyoyaka. Qa Dawid tawicaśta om śkanśkan śni yukanpi, hecen Saul iś makan hiwanka. Unkan Dawid tawicaśta kin nahmana heciyapi; Saul yakte yacin kinhan dehan oyakihi ce, eyapi. Tuka Dawid heya; Wakantanka tuwe wicaśtayatapi kağe cin he kiunniwaye kte śni ce, eya. Qa tawicaśta kin Saul kiunniye śni wicaśi. Hehan Saul wanke cin en iwahnana i, qa tawokoyake kin hee onśpa icu. He token on hecon kin kohanna nayahonpi kta.

Hehan iecadan Saul kikta, qa ohdoka kin etanhan kinanpa; qa ake taakicita om Dawid ode aya. Hehan Dawid iśs ohdoka kin etanhan inape ca, Saul kipan. Wicaśtayatapi mitawa, eya kipan. Hehan Saul hakikta, unkan Dawid Saul pakicapsan. Saul Dawid wanyag iheye cin on yuśinyaya. Unkan Dawid howahibayedan Saul okiye ca, He tokeca e cikte wacin kecanmayakin he, eciya: ohdoka wan ohna manke cin hen mahen yai ce. Unkan mitaoyate kin nikte maśipi, tuka ecamon 
śni ce, eye ça tawokoyake onśpa qon he kipazo, qa, Ate de wanyaka wo; nitawokoyake kin etanhan de iwacu ce, eya. Unkan cikte owakihi sni he. Hiya, owakihi, tuka hecamon wacin śni ce. He tokeca e omayade he. Wakantanka awanmayaka ce, heon kiunnimayeye kte śni ce, eciya.

Dawid hena heye cin ayaśtan kin hehan Saul iś heya; Dawid micinkśi he niye he, eye ça hankeya ceya. Eya wancadan Dawid waśtedaka, unkan ake dehan waśte kin piya hin sdonya; unkan iye kin śicaya econ kecaniçin, tuka Wakantanka en e iyopeiçiye śni.

Hehan Saul heya; Niye niwaśte kin he iyema. cece śni, qa mayakte yacin śni e sdonwakiya ce; qa anpetu wan en wicaśtaniyatapi kta ce, eya. Hecen anpetu kin he en Dawid kte wacin qon enakiye ca tiyata kiyotanka.

Tuka Dawid Saul weceye śni, hecen ti kin ekta ye śni. Ecin he itokam cinhintku Jonatan heya wahoya; tuka taku eye cin okipe śni, qa ake Dawid kte wacin, heon etanhan Dawid iś ake imnija ohdoka qon ekta e ki.

Saul Wakantanka iyokipiye śni kin hena e on tohinni iyopeiçiye sni, qa wahtani kin hena kicicajuju kta icekiye śni; qa tohinni Woniya Wakan kin da śni. Hecen ake kohanna Saul cante kin wakan śica yuśica ece.

Iho, micinca taku sica ecanonpi kin on wan- 
cahna. Wakantanka nicicajujupi, qa Taniya Wakan niçupi kta kida po. Hecanonpi śni kinhan wakanśica sanpa niyuśicapi kta.

\section{WOONSPE 12.}

DAWID QA SAUL TAWAHONKEZA.

Saul Dawid ode qon he ayuśtan nayahonpi qon. He iyohakam ake ecahankeya Saul Dawid śicedaka. Anpetu wan en wicaśta śicapi kin Saul en ipi, qa Dawid tukten naiçilibe cin he okiyakapi. Dawid takudan kiunniye śni, qa ohinni tanyan econ, tuka wicaśta wacin hinyanza hena Saul wokiyakapi.

Saul Dawid tukten un nahon kin heon iyuśkin, qa akicita ota om ekta ya. Tuka tukte etu hin un kin tanyan sdonye śni, tuka hen ikiyedan naic̣ihbe cin he Saul sdonya.

Unkan Saul taakicita kin paha wan akan eti wicaśi; qa tipi kin ihdukśan maka qe wicaśi. Okinni tokiyatanhan tuwe takpe wicahi kta kecinpi heon ; qa hanyetu eca tipi kin ohna kiwankapi, qa anpa eca hehan ake Dawid odepi ece.

Dawid ake Saul ode $n$ kin nakihon qa icanśica. Tuka Dawid tanyan wicada śni ; qa iye tawicaśta kin ekta iwanyag ye wicaśi. Unkan iwanyag ipi kin hdipi, qa Saul paha akan ahiti, hośi kahdipi. 
Hehan Dawid tawaśitku kìn etanhan om ekta ya. Qa ahitipi kin ikiyedan ipi qehan Dawid hewicakiya; Tuwe kici tiwehna mde kta he, eya. Unkan tawaśitku wanji; Miye unye kta ce, eya. Qa he kici hanyetu hehan tiwehna yapi. Maka oqe kin hena ehna yapi, tuka tuwedan wanwicayake śni. Oyate kin nina wiciśtinma, Wakantanka he iśtinbe wicakiya, hecen Saul awanyakapi qon hena eepi nina iśtinmapi.

Unkan wakeya kin tukte wanji en Dawid i kecanni he. Saul tawakeya kin he mahen i. Unkan Saul owinja ohna wanke cin ipahin en tawahonkeza pahdi han, qa makajanjan mini ohna nakun en han. Qa Saul awanyakapi qon hena eepi ihdukśan iśtinma hiyeya. Saul ti kin timahen Dawid hinajin kin ecaca sdonkiye śni.

Hehan Dawid tawaśitku qon heya; Wahonkeza kin de iwacu qa Saul wakte nun he, eya. Tuka Dawid heya; Kte śni wo. Wakantanka tuwe wicaśtayatapi kag̉a eśta ktepi kinhan he śica ce. Hecen iye Wakantanka tohan cin kinhan hehan te kta ce. Tuka miye tewaye kte śni ce. Hecen wahonkeza qa makajanjan mini ohnaka kin dena e unkicu qa unkahde kta, Dawid eya.

Hecen hena yuha tiwehna hdapi; tuka tuwedan kikta śni ecen kinapapi.

Hehan Dawid paha wan tokeca akan inajin; panpi tanin ihehanyan, qa awitukadan oyate kin 


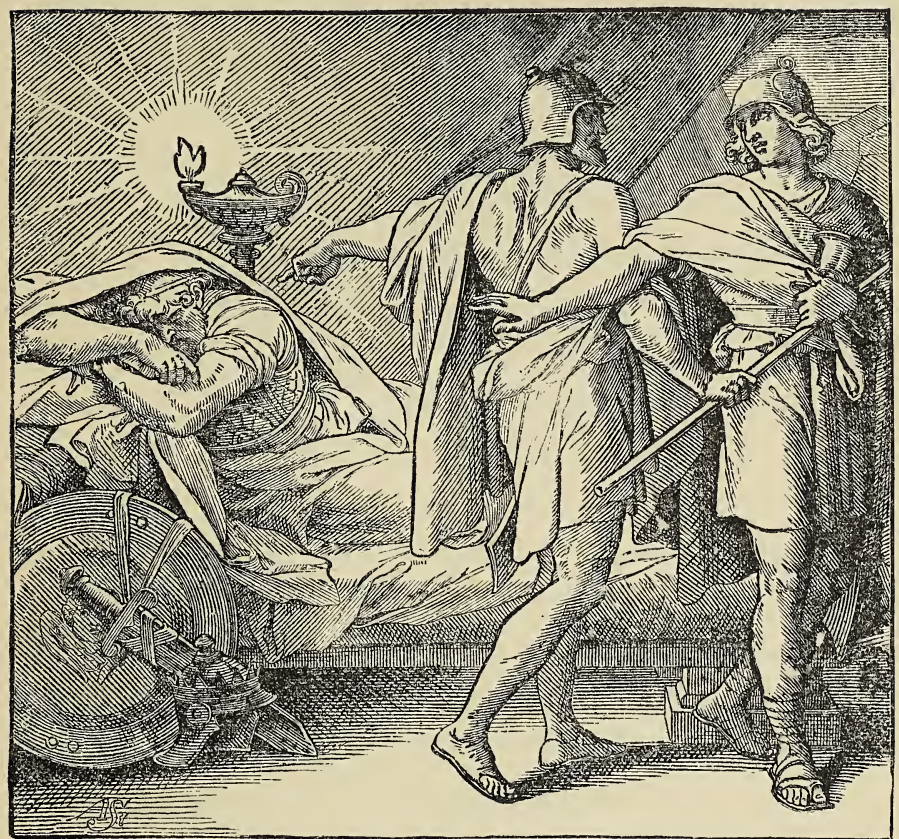

DAWID SAUL TAWAKEYA EN I.

wicakipan, qa; He tokeca wicaśtayatapi awandakapi kin oyate tokeca en upi tuka iyowinwicayakiyapi he, eya.

Token on he waawanjakapi kin owasin iśtinmapi kin sdonyayapi; Wakantanka he iśtinbewicaya.

Hehan Dawid wahonkeza qa makajanjan ko aki qon hena oyate kin wicakipazo.

Hehan Saul Dawid ho kin nahon, unkan kiksu- 
ya iheye ça ceya. Qa, Micinkśi Dawid ho kin hee he, eya. Unkan Dawid heya: Wicaśtayatapi itancan mitawa, he miye maho ce; he tokeca e nakun omayade he; śicaya ecamon he; qa wicaśta śice cin cikte wacin, keya oniciyakapi he, eya.

Hehan Saul heya; Miye wawahtani ce. Anpetu kin de mayakte śni, heon etanhan icimana kiunniciya wacin kte śni ce, tiyata hda wo, eya.

Saul taku śica econ kin on iyopeiçiya he. Hiya, iyopeiçiye śni, qa Wakantanka kicicajuju kta e wocekiye eye śni.

Hehan Dawid, Wicaśtayatapi tawahonkeza qon dee ce, ookiye wanji huwe uśi po, eya.

Unkan he tokeca Dawid iye atayedan wahonkeza kin ekta kicicaye śni he.

Hehan Saul heya; Micinkśi Dawid, niye niyawaśtepi ce, eye ça hehan kihda. Unkan Dawid iś Kanan en ahankeya Pilistim tonwanyanpi heciya i ; okinni heci un kinhan Saul ode kte śni kin heon. Unkan Wakantanka Pilistim oyate kin Dawid cantekiyapi wicakagga.

Dawid Pilistim oyate kin om un kta iyecece śni tuka. Ecin oyate kin he wakaggapi ohodapi kin heon.

Unkan Saul hantuqe taku śica econ kin he ayuśtan kta on Wakantanka icekiya he. Hiya, hecon unkanś Wakantanka okihiye kta tuka.

Iho, hokśipidan, wicinyanpidan, ito he ake ca- 
jemdate kta. Taku śica ecamon kin he amduśtan kta, ehapi eśta, nicantépi on Wakantanka ceyakiyapi śni kinhan oyakihipi kte śni. Ecin wicacante. kin en wakanśica u kte cin waśaka. Heon etanhan iyopeniçiyapi kin cante on econ po, qa Woniya Wakan kin nina da po, he niyuwaśtepi kte cin hee.

\section{WOONSPE 13.}

DAWID QA WAHOYAPI YUECETUPI KIN.

Hehan Dawid Pilistim totonwepi kin ekta i nayahonpi qon. Dawid tawaśitku kin om i; qa oyate kin he sicapi qa wakagapi ohodapi heca eśta, Dawid tawaśitku om cantewicakiyapi Wakantanka wicakağa.

Hehan Saul Dawid Pilistim oyate kin ekta wicai, nalion kin hetanhan icimana ode śni. Ecin oyate kin he ehna Dawid ode eśta, oyate kin he ktepi kte cin heon etanhan.

Pilistim oyate Israel śicewicadapi qa om ohinni tokakiciyapi nayalionpi qon. Ake anpetu wan en Pilistim wicaśtayatapi kin akicita ota iwicacu, qa Israel takpe wicaya. Saul he nakihion qehan is eya taakicita om itkowicakipa. Saul cinhintku Jonatan nakun opa.

Qa hecen om kicizapi. Unkan unma tukte $3^{*}$ 
ohiyapi he. Pilistim e ohiya. Ecin Wakantanka Israel canniyewicaya, heon owicakiye śni ; qa hecen iyayewicayapi, qa ota ewicaktepi. Qa Saul cinhintku Jonatan nakun ktepi. Qa Saul iś kaken

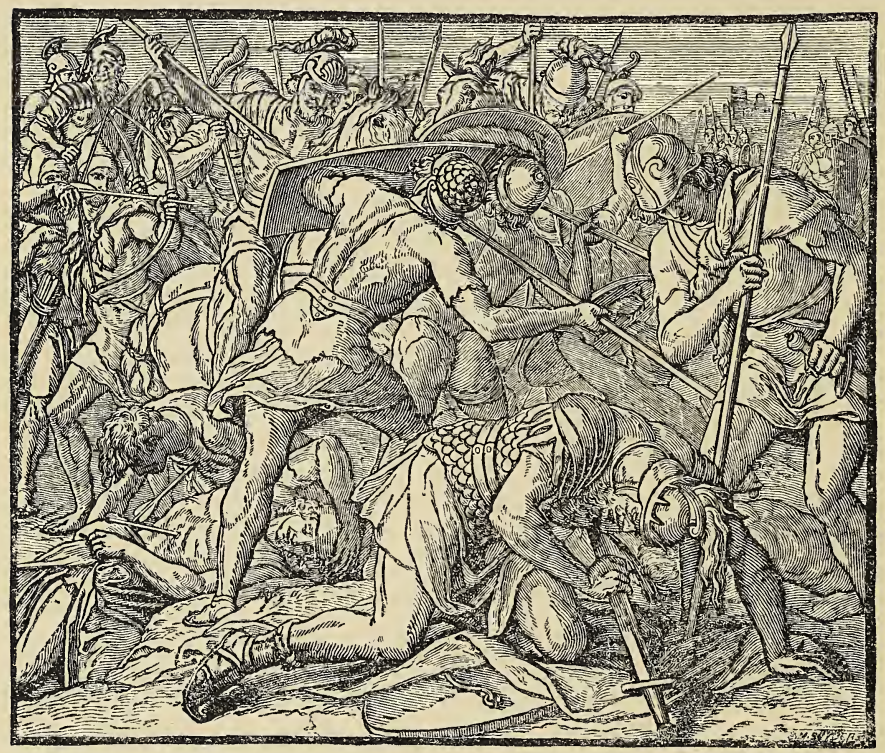

SAUL ICIKTE.

ta. Wanhinkpe ece on opi, qa nina taopi, hecen tokadan najica okihi śni; hehan Saul nina canśica, te śni ecen yuzapi kinhan śikśicaya kuwapi kte cin heon yuzapi śni itokam ța cin; qa iye taakicita kin wanji heciya; Mazasagye icu qa camapa wo, eya. Unkan akicita kin he Saul kte kta iyececa he. Niśs nitakuyepi wanji heniciyapi ehan 
yaktepi naceca he. Hiya, ecanonpi kte śni. Tinwicayakte kte śni, Wakantanka eya.

Saul taakicita econ śni; unkan Saul iye tamazasagye ikikcu, qa caic̣ipe ça içikte. Saul țe cin iyohakam akicita qon hee iś eya mazasagye ikikeu qa içikte. Saul hecon wanyake cin heon iś econ. Wicaśta tona śicapi kin hena heconpi. Tuka tona Wakantanka waśtedapi kin hena țapi kta eśta Wakantanka e apepi.

Okicize kin ihanlianna qehan Pilistim Israel wicaktepi kin tancanpi kin owicadepi; unkan Saul cinkintku Jonatan kici tancanpi kin iyewicayapi. Unkan Pilistim he nina iyuśkinpi, wicaśtayatapi kin cinca kici ktepi kin heon. Tuka wicatancan to eśta ake nakun śicaya kuwapi kin he wowiśtece.

Saul pa kin baksapi qa tawokoyake kin nakun owasin icupi. Saul tawokoyake kin hena maza on ikicize kin heca. Unkan Saul pa qa taheyake ko otonwe iyaza ayapi; qa iye wakağapi yuhapi kin nina hdatanpi, qa heyapi; Unkiye wakağapi unhapi kin de Israel tawakantankapi kin isanpa tanka ce, eyapi. Hehan Saul taheyake qon wakag̉api tipi hdepi kin en ehnakapi, qa tancan kin nakun en okatan ehdepi, qa Jonatan tancan kin nakun heconpi. Hecen oyate kin owasin wanyakapi kte cin on heconpi. Tancanpi kin oiyokisinya wicakuwapi; tuka Jonatan Wakantanka waśtedaka, 
hecen naği kin Wakantanka kici un; tuka Saul e naği kin Wakantanka kici un śni.

Israel he nahonpi, Saul cinhintku kici okatan wicahdepi kin he; unkan Israel etanhan wicaśta waonśida wanjikji hanyetu en ipi, qa wicayulipapi qa wicahapi.

Dawid iś he ehan Pilistim tamakocepi kin ahankeya un, qa okicize kin he sdonya. Hecen unma tukte ohiyapi kin he nahon kte hinca. Unkan unhanketa wanji Dawid ekta hi. Wicaśta kin he Dawid wanyake çehan pakicapsan. Hehan Dawid heya; Tokiyatanhan yau he. Unkan wicaśta qon is ; Israel tipi kin heciyatanhan wau ce; kicizapi tuka Israel nakipapi, qa ota wicaktepi, qa Saul cinhintku kici nakun wicaktepi ce, eya.

Wicaśta qon he Saul ktepi okiyake cinhan, Dawid iyuśkin kta kecin. Tuka Dawid iye qe Saul waśtedaka, qa ța nalion kin on iyokiśica; qa takodaku Jonatan on nakun Dawid nina iyokiśica; qa Pilistim ohiyapi kin he nakun on. Ecin he ohiyapi kin on wakağapi yuhapi kin nina hdatanpi, qa Wakantanka e sanpa ohodapi śni kin hena on Dawid icanśica.

Hehan Dawid odowan wan waste ahiyaya; Saul qa Jonatan he iwicadowan, qa hecen eya; Saul qa Jonatan wanmdi qa mnaja iyecen waditakapi ce. Napin tipidan qa napin țapi ce. Qa akeśs hecen eya; Mihunkawanji Jonatan, tema- 
DAWID QA KOKA KIN ZION PAHA AKAN.

yaliinda kin he tanka qon, dehan on makakija, eya.

Dawid Saul yaśice śni, tuka yawaditaka ecepan. Wicaśtayatapi kin heon taku śica eciye kapin. Dawid wicaśtayatapi kte cin wanna iyehantu; heon Wakantanka Israel ecen cante yuswicakiya. Hecen Israel Dawid wicaśtayatapi dapi. Hehan Dawid iś wicaśtayatapi kta kewicakiya. Ecin Wakantanka Dawid hecen wahoya.

Wakantanka taku iwahowicaye cin tohinni akiktonje śni. Wakantanka Abraham hecen iwahoya; Cinca yuke ça sanpa cincapi kin Kanan makoce kin en ounyanpi kta, iwahoye ça econ.

Iho, micinca, niś Wakantanka Taniya Wakan kida po. He iwahoniyanpi kin, nina yakidapi kinhan niçupi kta.

Hehan Dawid candowankiyapi on Wakantanka yatan dowan ; taku iwahoye ciqon wanna iyekicihantu kin heon. Qa wicaśtayatapi un kinhan ohinni tanyan econ, qa oyate kin owasin Wakantanka ecedan waśtedakapi kta onspewicakiya cin.

\section{WOONSPE 14.}

DAWID QA KOKA KIN ZION PAHA AKAN.

Wakantanka Dawid wicaśtayatapi kag̉e cin he iyohakam otonwe wan Jerusalem eciyapi, hen 
Dawid ti. Bethlehem otonwe kin hen Dawid tonpi.

Jerusalem otonwe kin he owaśtecaka; qa he en paha tankinkinyan ota. Paha kin he wanji Zion Paha eciyapi.

Zion Paha kin akan tipi wan waśte kağapi kta Dawid cin, qa he wicaśtayatapi tipi kta

Dawid Wakantanka nina waśtedake cin heon, Wakantanka Koka tawa qon he iye ti kin ikiyedan yanke kta cin.

Koka qon he eya Śiloh ekta yanka. Unkan okicize ekta aipi; qa iyoopta Pilistim akipi. Tuka ake Israel ekta ki, nayahonpi qon. Hehantanhan tohinni Ś́loh ekta ayapi śni. Ecin Śiloh en oyate unpi kin he śicapi, heon etanhan Wakantanka icimana Koka kin hen yuhapi kta cin śni. Koka qon tokiya yanke cin Dawid he sdonya. Hecen iye hinca huwe i. Wośnapi itancanpi qa oyate wicota Dawid kici ipi. Qa cotanka qa candowankiyapi ocaje ota on dowanpi. Qa apa iyotan odowan waśte ahiyayapi, Psalm odowan kin he on Wakantanka yatanpi. Qa winohinca kin iś eya nakun candowankiya on dowanpi. Hecen Koka kin he Jerusalem en Zion Paha ekta akupi. Dawid qa wośnapi itancanpi, qa oyate kin tona candowankiyapi kin owasin wokoyake ska qa hanska ece koyakapi. 
DAWID QA KOKA KIN ZION PAHA AKAN. 63

Unkan hena hecen koyakapi qa Wakantanka yatanpi kin he oiyokipi hinca.

Niś dena iyececa wandakapi ehan, iyotan mahipiya ekta Wakantanka taohnihde unpi kin hena ekta ewicawacannipi naceca he.

Oiyokipi kin heon wicota wawanyakapi. Anpetu kin he en Dawid nina wiyuśkin.

Hehan Koka qon he Zion Paha kin ekta ayapi.

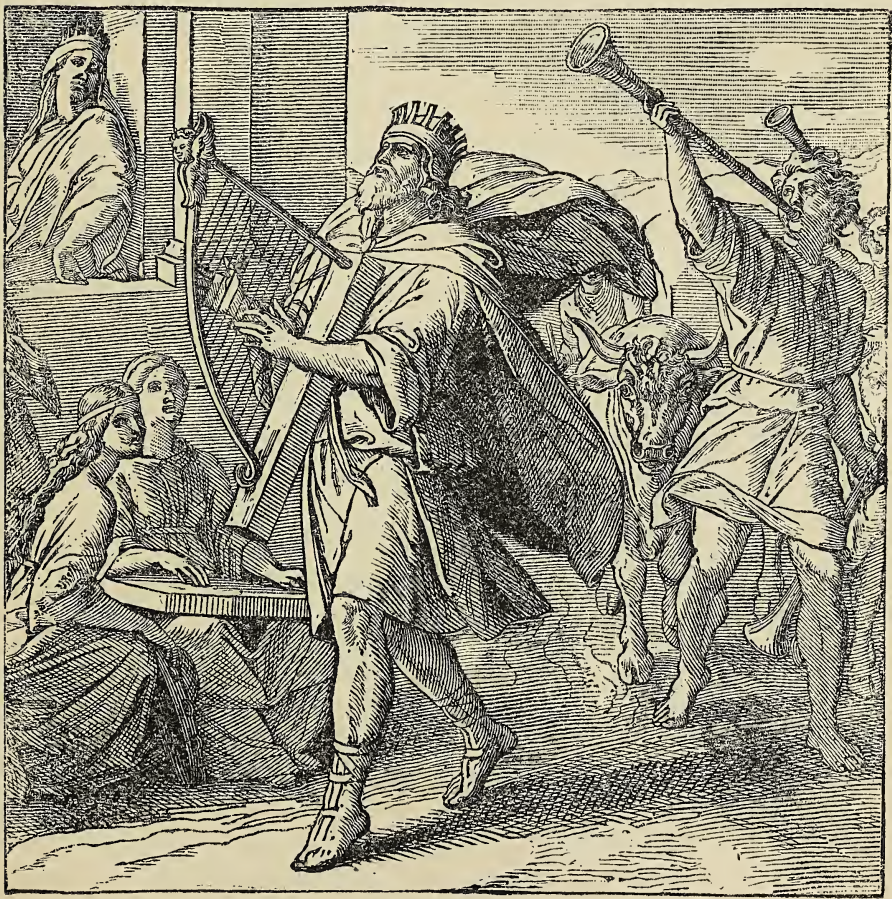

DAWID KOKA WAKAN KIN AHDI. 
Qa Zion Paha kin he acankaśkapi. Qa conkaśke tiyopa tanka kin yuzamni ehdepi. Qa wicaśtayatapi kin Koka kin ahna tin ayapi, qa wośnapi itancanpi kin hena koya. Qa Dawid ti kin icahda wośnapi Wokeya wan kahwicakiye ça Koka kin hen ayapi kte cin he nakun ozanton wiyeya hdepi. Hehan wośnapi itancanpi kin hena eepi Koka kin tin aipi. Kehan Dawid Psalm wanji en, Itancan wopida qu po, eyapi kin he ahiyaye wicaśi.

Hehan Dawid Zion Paha kin akan Wakantanka wakiyuśna. Qa oyate ihdukśan najinpi kin wicayawaśte ; qa wica qa winohinca otoiyohi ag̉uyapi tado qa miniśa ko wicakipamni. Dawid waonśida hinca.

Hehan Dawid nina iyuśkin; Wakantanka Koka tawa kin he iye ti kin ikiyedan yanke cin heon. Qa Koka kin en ikiyedan, anpetu hanyetu ko, candowankiya on dowanpi kta Dawid cin. Apa anposkantu eca dowanpi, qa apa iś hanyetu ekta dowanpi ece. Malipiya kin ekta mahpiya ohnihde unpi kin hena ozikiya wanin ohinni dowanpi. Tuka wicaśta akantu kin eqe hecen econpi śni. Hecen Dawid iye ti kin en yanke cin ohinni Wakantanka idowanpi kin he nahon; qa hanyetu oġunga eca nakun oiyokipiya dowanpi kin nation wanka ece.

Psalm odowan kin hena Dawid tadowan. Qa Wakantanka Taniya Wakan kin he Dawid onspe- 
DAWID QA KOKA KIN ZION PAHA AKAN. 65

kiya. Hecen token eye kte cin owasin onspe, qa ecen owa, qa dowanpi kin hena wicaqu. Hecen ohinniyan Koka kin ikiyedan dowanpi.

Tuka Dawid iye kin ohinni owanji yanke śni. Pilistim qa oyate kin tona sicapi kin hena wicakis i; qa Wakantanka okiya. Hecen okicize kin ohinni ohiya ece. Qa tohan owanji hdiyotanka eca, hehan Wakantanka okiye ça ohiye cin heon yatan qa idowan ece.

Anpetu wan en Dawid takodaku Natan kici yanka. Unkan Dawid iye tipi iyotan waśte wan oti, tuka Wakantanka Koka tawa kin e ozantonpi kin hecehnana he cin, heon etanhan tipi wanji waśte kicagge kta cin.

Dawid takodaku Natan he wicaśta ksapa qa waśte; unkan Dawid tipi wakan wanji kagge kta cin kin iyowinkiya.

Unkan hanyetu wan en Wakantanka Natan heciya; Dawid tipi wan micagge kte cin econ kte sni ce, he oyakidake kta ce. He micage kta cin kin iyomakipi ce. Tuka tipi micag̀e kta wakahinige cin hee śni ce. Okicize nina econ qa wicaśta ota teye cin heon etanhan. Tuka Dawid cinca wan waqu kta ce; kinhan tipi micage kte cin hee kta ce. Qa Dawid iye kin okicize econ kta ce; qa ohinniyan mdawaśte kta ce.

Hehan ihanlianna qehan Natan Dawid en hi, qa Wakantanka-taku eciye ciqon he Dawid okiciyaka. 
Dawid he nakilion kin nina iyokipi; Wakantanka yawaśte, qa cinca wan qu kta, qa hee tipi wakan kaġe kta iwahoye cin heon; qa Dawid Wakantanka wopida eciya.

Qa heya; Jehowa cantemayakiye cin he tanka ce. Ake sanpa mayadawaśte ce; wopida ota kin on token epe kta he. Heciciya ce; Ohinni mayawaśte qa waśte madaka ye.

Dawid tawocekiye kin Wakantanka iyokipi. Dawid iś he itan śni; tuka Wakantanka cantekiye cin he nina awakicin. Ecin iye kin wahtani kin on onśiic̣ida, qa iye waśte qa on etanhan Wakantanka yawaśte kecanic̣in śni; tuka yawaśte qa cinca wan yawaśte kta iwahoye cin he awakicin kin tanka.

Unkan unkiye Wakantanka canteunkiyapi śni he. Qa taku unkidapi eśta econ kte śni he. Hiya, econ kta; unkiye unwaśtepi on śni, tuka iye waonśida waśte kin on etanhan oyate wicayawaśte kin iyokipi.

\section{WOONSPE 15.}

DAWID QA URIAH TAWICU QON.

Dawid wicaśta waśte qa Wakantanka iyokipiya cin. Unkan taku on he Dawid waśte he. Wakantanka Taniya Wakan Dawid cante kin en 
ehnake cin heon etanhan hececa. Tuka Dawid waśte nayahonpi kin heon tohinni wahtani śni kecannipi nace. Hiya, hececa tuka tuwe nina awanihdake śni eca iśs eya woniya śica cante kin iyohpaya ece. Unkan Dawid tuktekten Wakantanka cekiye cin ayaśtan ece; unkan hecen wakan śica iyutanyan.

Unkan oyate wan śicapi e Kanan makoce kin ikiyedan tonwanyanpi he wicakis ye kta. Tuka Dawid iye hinca ye śni. Tuka wicaśta wan Joab eciyapi he itancanyan ye śi. Qa Dawid. iye Jerusalem en owanji yanka. Tuka token on he iye hinca ye śni kin sdonwaye śni. Tuka okinni iye kin owanji yanke ça wote ça wayatke ça iyokipiiciya cin on hecon naceca.

Anpetu wan maśte, unkan Dawid diditahda, qa iye tohe kin akan wanka. Qa hehan cusni qehan, tipi kin he akan tanhan kin mdaskaya kagapi qa he en omani ece. Unkan ake he en omani, unkan icunhan winohinca wan ihukuya hiyaya wanyaka. Unkan winyan waśtedake hinca, qa taokiye wanji ekta ye si, qa he tuwe hecinhan iwanğe śi. Hecen ekta iwanh i, qa winyan kin he Batśeba eciyapi, qa hihnaku kin iś Uriah eciyapi.

Dawid winyan kin de nina iyutanyan. Hecen on Wakantanka cekiye ca taku iyutan kin he tokan iyekiciciye kta icekiya iyececa tuka. Hecen 
winolinea kin he e ohinni awacin un ; qa hihnaku ta unkanś, hehan mduze kta tuka, ecin. Uriah iś he icunhan kicizapi kin ekta opa. Wicaśta waśte qa waditaka. Hecen Dawid, tokin Uriah okicize kin ekta ktepi qeś, ecin.

Ecen unhanketa woniya śica Dawid cante kin iyohpaye ca iye taakicita tokapa kin Joab wowapi kicağe ça heya; Tohan kicizapi kinhan, Uriah tukten ktepi kta idacin kinhan hen ye yaśi kta ce, eya kicağa.

Dawid Joab śicaya econ śi kin anag̉optan śni iyececa. Tuka Joab iś wicaśta śica, heon etanhan econ kte cin itonpe śni. Qa he ecahankeya oyate wan cankaśka yanka e kizapi. Unkan Joab Uriah akicita qeya om conkaśke kin ikiyedan ye śi. Qa ecen econ, tuka en ktepi.

Hehan Joab Uriah ktepi kin Dawid ekta okiyag yewicaśi. Qa Dawid he nahion kin on iyokiśice konza; tuka cante kin ekta e he iyuśkin, tuka hececa. Qa hehan Batśeba huweye wicaśi, qa hecen yuza.

Dawid ohan kin Wakantanka nina śice daka; qa Wakantanka Natan okiyaka. Natan he wicaśta waayata heca.

Unkan Natan Dawid en i, qa ikce wicowoyake wan okiyaka, qa heya; Wicaśta nom otonwe wanjidan en tipi. Unkan unma wijica, qa unma iśs wahpanica. Qa wijica qon he talinca wanunyanpi 
ota yuha. Qa wahpanica kin iś talinca wanunyanpi cinca wanjidan yuha; tuka toka icaga ehantanhan yuhe ca tanyan kicuwa; iye tawoyute qa tawoyatke kin hetanhan yunkiye ça yatkekiya; qa cinca teliikekida kin he iyececa waśtekidaka; qa iye maku kin hen ohna hduha. Unkan wicaśta

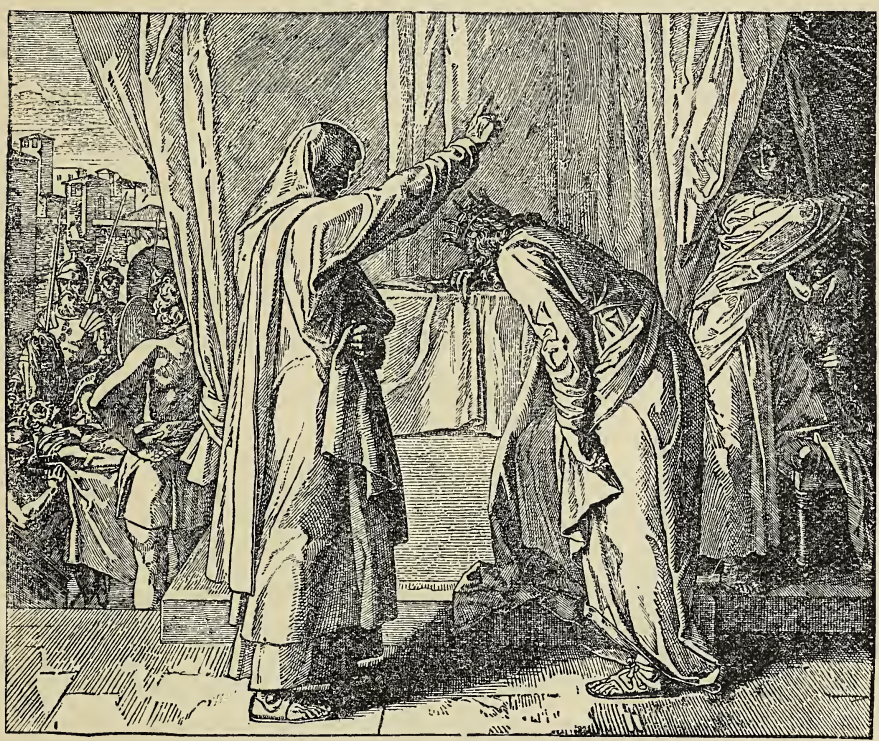

NATAN DAWID IYOPEYA.

wijica qon he anpetu wan en ti kin en titokan ai. Unkan wijica qon he unma wahpanica tahinca wanunyanpi cincadan wanji yuhe ciqon he huweye wicaśi, qa he kte, qa iye ti en titokan ahi qon he yunwicaya, eya. 
Iho, hecen wijica qon he token ecakiconpi kta iyececa he, Natan eya.

Dawid he nahion kin nina śicedaka, qa heya; He ikicu kin etanhan talinca wanunyanpi tom qu, qa hehan iye kin nakun țe kta ce, eya.

Hehan Natan, Wicaśta wijica qon he niye ce, Dawid eciya.

Natan Dawid śicaya econ kin he kicipazo cin kin heon etanhan wicowoyake kin he okiyaka.

Hehan Natan Dawid heciya; Wakantanka canteniciye ça wicaśtayatapi nicağa; unkan he tokeca e tawoahope kin anayagoptan sni he. Heon Wakantanka kakiśniye kta ce. Nicinca kin iyakitedan tokakiciyapi, qa kiciktepi kta ce; qa tohanyan ni yaun kin hehanyan niciyuśepi kta ce, eya.

Dawid he nahon kin nina iyokiśice ça, Wakantanka en wawalitani ce, eya.

Unkan Natan heya; Wayahitani kin Wakantanka nicicajuju ce, nițe kte śni ce eciya.

Saul wikopa eceedan on Wakantanka ekta ewacin; tuka Dawid e qe wahtani kin ocowasin iyokiśica.

Hehan Dawid iyokisice cin he ecen odowan wan ahiyaye ca, dowampi kin he wicaqu; hecen Wakantanka Koka tawa kin icahda odowan kin he ahiyayapi.

Dawid tadowan kin he en kaken eya; Wa ska 
kin iyecen mayujaja, qa cante teca makagga wo. Wakantanka Nitaniya Wakan ake maqu ye.

Dawid wahtani kin he Wakantanka kicicajuju kta icekiya; unkan kicicajuju. Qa hecen wakansica ti kin en iyaye śni. Tuka Dawid țe śni kin itokam Wakantanka iyopeya. Hecen woahtani kin he Wakantanka sicedake cin oyate sdonyapi kta.

Tho micinca, Wakantanka taku wawiyutanyan owasin etanhan enihdakupi kta nina icekiya po. Taku sica ecanonpi kin hena on Wakantanka en iyopeiçiya po, kinhan nicicajujupi kta ce. Waunhtanipi kin heon Jesus țe ça, heciyatanhan woiyopeiçiye econ unśipi ce.

\section{WOONSPE 16.}

DAWID, QA IYOPEYAPI KIN.

Dawid ni un kin icunhan Wakantanka iyopeye kta keciya e nayahonpi qon.

Dawid wanna cincota, tuka cinca kin apa śica. icag̉api. Tuka hena otoiyohi owicamdake kta owakihi śni. Cinca kin wanji Absalom eciyapi. Unkan kośka kin he witantanka, owanyag waśte, qa paha kin nakun waśteśte kin he itan; qa nakun ecen itonśni, qa iye hunkawanjinwicaye cin wanji cante okiçunninya, unkan kikte. 
Dawid he nation kin on Absalom nina canniyeya, qa tehan wanyake śni un. Tuka unhanketa iye ti kin en u kte cin iyowinkiye ça hehan wanyaka. Unkan i iputake ça canniyeye ciqon he kicicajuju. Absalom hunkawanjitku kikte kin heon etanhan otonwe kin hen $u$ kte cin Dawid he iyowinkiye kte śni iyececa tuka. Hecen cinca kin nina waśtekidaka.

Tuka Absalom iye kin atkuku waśtekida śni; qa ecen atkuku kin wicaśtayatapi ki wacin; qa oyate kin ehna waśte kiçun un. Hecen oyate kin waśtedakapi kinhan iye wicaśtayatapi kag̉api kta he cin. Qa tona onśika wanwicayaka eca i iwicaputake ça; Wicaśtamayatapi unkanś canteciciyapi kta tuka, ewicakiya ece.

Absalom hecon kin he wicaka kecinpi, qa on oyate kin waśtedakapi. Tuka Absalom wicaśtaśni wicahnaye cin he Wakantanka sicedaka.

Unkan wanna Absalom oyate kin ota waśtedakapi sdonkiya, hehan atkuku Dawid en i, qa ma. koce tokanta ye kta keya iwanga. Unkan Dawid is iyowinkiya. Absalom taku kuwa kin Dawid he sdonye śni.

Hecen Absalom Jerusalem etanhan iyaya. Wicaśta opawinge nonpa owicapa, qa makoce ekta ye kte cin he kohan toktokeca ekta ye wicaśi qa hewicakiya; Tohan cotanka yajopi nayahonpi kinhan, Absalom wicaśtayatapi ce, ehapi kta ce, 
eya. Hecen wanna makoce kin he en i qehan, cotanka yajopi ; unkan, Absalom wicaśtayatapi ce, panyanhan eyapi. Unkan oyate kin wicota Absa. lom en hipi, qa hena akicita wicayuha.

Absalom wicaśtayatapi ic̣icag̉e cin he Dawid ekta hośi kaipi.

Dawid he nahon kin nina icanśica; cinhintku ohan śice cin he awacin yanke kte cin śicedaka. Dawid iye wahtani kin on kakije cin he hecetu içidaka, qa Wakantanka he woiyokiśice kin hena akipeye cin he sdonkiya.

Unkan Dawid Jerusalem en yanka eśta, okinni Absalom hen u qa kte kta kecin; qa hecen Zion Paha kin akan tipi waśte oti qon hena ehpeya icunonpa ya. Unkan oyate kin tona Dawid waśtedakapi kin hena ihaktapi qa kici yapi.

Unkan Jerusalem etanhan yapi, qa tokaheya wakpadan cistinna iyuwegapi. Unkan oyate kin owasin ceyapi; wicaśtayatapi qon ti elipeya ti codan ye cin he awacinpi qa on ceyapi.

Hehan Dawid taoyate om paha wan tehanwankantu ekta ya. Dawid pa kin aihdahpe ca sicodan ceya ya; he iyokiśice cin heon hecon. Unkan oyate owicape cin owasin iś eya iyecen econpi, qa ceya yapi. Oyate kin Dawid nina waśtedakapi. Hehan Dawid paha kin akan i qehan Wakantanka cekiya. Dawid kakiśya un eśta Wakantanka he kicanpta okihi e sdonkiya. 


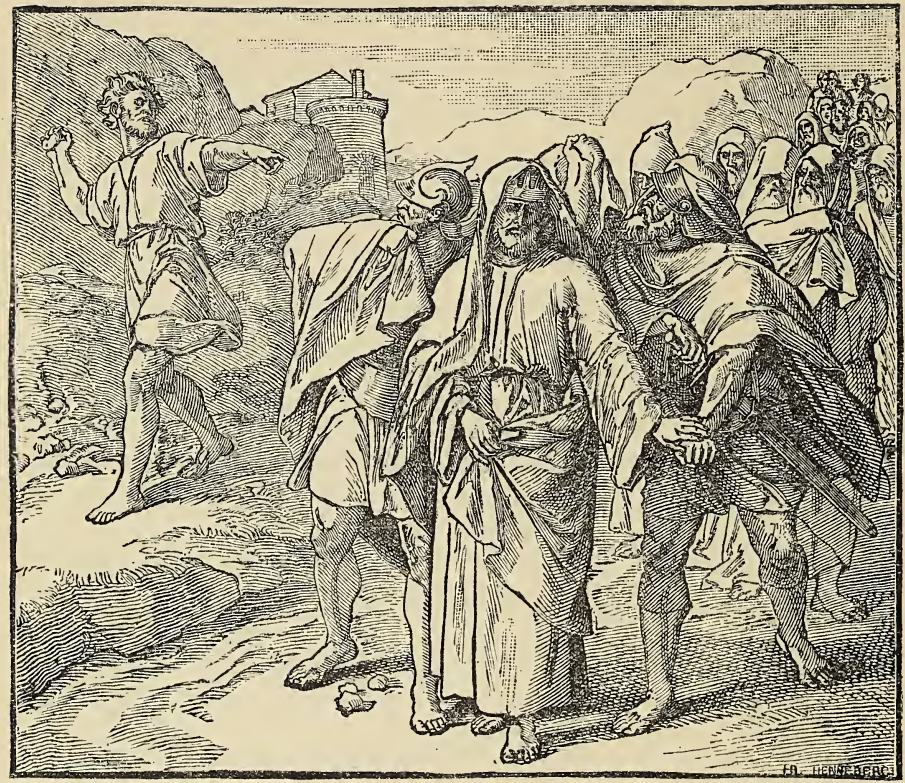

SIMEYI DAWID YASICA.

Hehan ake iyoopta yapi. Unkan wicaśta wan śica e Dawid akipa. Wicaśta kin he Dawid sicedaka, qa ośtehda, qa inyan on kinin, qa Dawid taoyate kin nakun wicakinin; qa heya, Wakanśica nicinca ce. Saul cinca om wicayakte qon heon dehan Wakantanka kakiśniyan ce, eciya. Unkan Dawid Saul cinca om wicakte he. Hiya, wicaśta kin he Dawid oitonśni. Tuka e qe Dawid Uriah țeye ça hecen walitani kin heon Wakantanka kakiśya. 
Unkan Dawid tawaśitku kin wanji heciya; Wicaśta śice cin he niyaśice cin ijowinkiye śni wo. Qa ito miye pa wakakse kta ce, iyowinmakiya wo, eya. Tuka Dawid heya; Hiya, he mayaśice cin Wakantanka iyowinkiya ce; hecen ayaśtan waśi kte śni ce. Miye micinkśi e iśin makte kta omade kta ce; heon wicaśta kin de mayaśice cin iyomayake kte śni ce, eya.

Wicaśta śicapi kin en Dawid walibayedan un kin de sdonunyanpi. Unkiyepi kin tuwe unyaśicapi kinhan token econkupi naceca he. Ùnkiś eya taku sica econkupi he awaunkicinpi, qa on etanhan taku hecen unkakipapi eśta unwahbapidan kta isececa.

Dawid taoyate kin om tehan ohnihdeya unpi, qa owasin nina mdokițapi hehan unhanketa Jordan wakpa kin iyuweğapi. Makoce kin he hewoskan makuce eyapi. Dawid taoyate om hen oziiçiya.

Unkan wicaśta wijica yamni makoce kin he ikiyedan tipi; ca Dawid taoyate om hen i kin he nahonpi, unkan hekiciyapi; Makotahedan unpi kin wotektehdapi, qa ipuzapi, mdokițapi ko nace, eyapi; qa taku ota wicakaipi, owinja qa wakśica, qa miniyatke, qa aǵcuyapi, qa wamnaheza, qa tulimağa canhanpi, qa asanpi suta, qa talinca wanunyanpi ko, yutapi kta wicakaipi. Dawid kakiśya un kin icunhan wicaśta kin hena cantekiyapi kin he Wakantanka hecen canteyuswicakiya. 
Dawid hewoskan makoce kin hen un kin nina wocekiye eya. Wakantanka wokicanpte kida. Iyotan iyekiya eśta heon woyanic̣iye śni, qa onśiicida un.

Iho, he iyecen micinca, niś taku śica ecanonpi, qa on iyopeniyanpi kinhan, sanpa śihdapi śni po ; tuka niśicapi kin on onśiiçiya iyopeiçiya po, qa Wakantanka cekiya po, hecen onśinidapi kta.

\section{WOONSPE 17.}

DAWID, QA UTUHU CAN KIN.

Dawid taoyate kin om liewoskan makoce kin en tonwanyanpi totonwepi kin ihdukśan conkaśke tonpi.

Unkan Absalom atkuku heciya un kin hehan nalion, unkan akicita ota om takpe u, qa Dawid totonwe kin ikiyedan ahiti.

Unkan Dawid cinhintku sice cin he takpe hi kin wanhdake ça iśs taakicita om ye kta cin. Tuka Dawid iye hinca ye kte cin iyokipipi śni. Okinni okicize kin en ya eśta ktepi kte; heon owanji yanke sipi. Oyate kin he Dawid nina waśtedakapi. Unkan Dawid, Token yacinpi kinhan ecen ecamon kta ce, eye ça hecen ye śni. Dawid iye kin cinhintku kici kicize kte cin cin śni.

Dawid taakicita kin yapi kte cin itokam cin- 
hintku Absalom kiunniye śni wicaśi. Dawid cinca kin śica eśta waśtekidaka. Hecen om kicizapi canwehna kicizapi, qa hecen ota can ibotopi, qa ksuweiçiyapi qa içiktepi.

Unkan kicizapi kin unma tukte ohiyapi he. Dawid taoyate kin ohiyapi, Wakantanka owicakiye cin heon ohiyapi. Absalom taoyate kin nakipapi; tuka ota ewicaktepi, qa ota nakun can iboto içiktepi.

Unkan Absalom iś śonśonna wan akanyotang napa; qa utuhu can wan tanka ihukuya iyaya, tuka paha kin hanskaska hinca, hecen can adetka wan ikoyake ça en kaozezeya otke, qa śonśonna qon eceedan napa. Absalom paha hanskaska waśteśte, unkan he wowitanyan e nayahonpi qon. Tuka Wakantanka hecece kta ecankin.

Unkan Dawid taakicita kin wanji Absalom hecen otke kin wanyaka, qa akicita tancan Joab he okiyaka. Unkan Joab heya; Tokeca e he yakte śni he; yakte unkanś mazaska ota wokoyake ko ciẹu kta tuka ce, eya. Tuka iśs ayupte c̣a heya; Mazaska kektopawinge mayaqu eśta Absalom kiunniwaye kte śni Dawid cin e nawahon ce, eya. Unkan Joab ainina akicita wikcemna om ekta i; qa Absalom otkeyahan iyeya. Unkan Joab wanhinkpe yamni icu qa Absalom cante kin cokaya iheya. Hehan akicita wikcemna om i qon hena iś eya mazasagye wahonkeza ko on ktepi. 


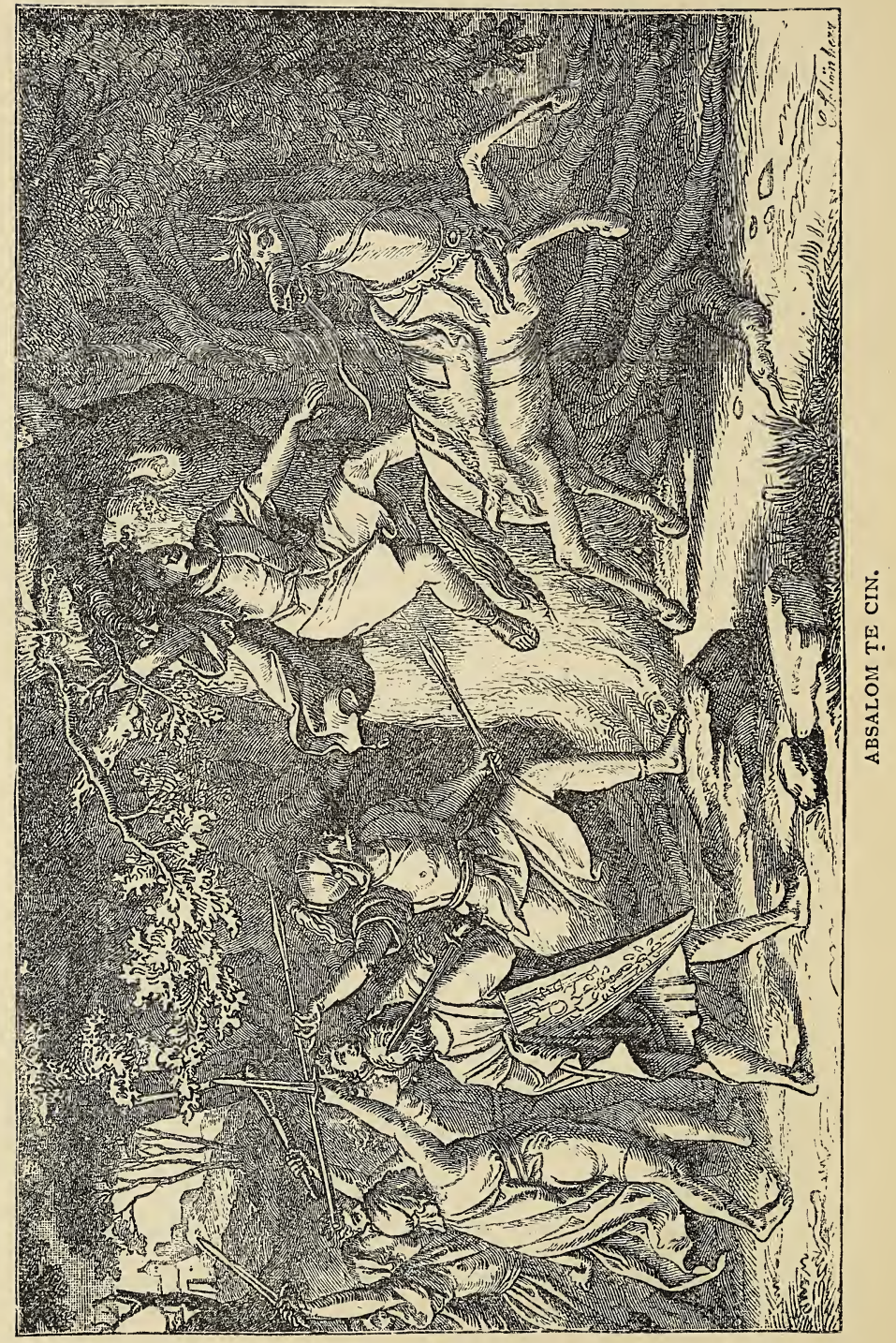


Hehan Absalom tancan kin yuhipapi qa makohidoka wan en ohnakapi, qa inyan ota iyahdaskinyapi.

Hehan Joab cotanka yajo, qa taakicita kin owasin wicakico. Wanna Absalom ța hecen kicizapi kin ayuśtanpi; qa Joab taakicita om Dawid totonwe kin ekta hdapi; qa Absalom taakicita kin iś iye ahitipi qon ekta kipi.

Joab nahanhin Dawid yanke cin ehan kipi śni, itokam nom kahintokam dus hdapi; Dawid wokiyakapi kte cin on. Qa Dawid iś wanahon cantokpani yanka; Absalom ktepi qa iśs ni unma tukte kin he. Dawid taoyate kin ohiyapi kte cin he cin ; tuka cinhintku kin he qe kiktepi kta cin śni.

Conkaśke kin mahentanhan, tiyopa kin icahda hen Dawid oyankeya, unkan hen yanka. Qa wanji iś conkaśke akan najin. Unkan waawanyake cin he, Wicaśta wan inyang u wanmdaka ce, panyanhan eya. Unkan Dawid iś, He wahośi u ce, eya. Tuka ake waawanyake cin, Ake nakun wanji tokeca ecen u ce, eya. Ake Dawid iś, He nakun wahośi u ce, eya.

Unkan tokaheya u qon e, Owasin tanyan kupi ce, eya. Qa hehan wicaśta qon hee Dawid itokam ite makipusdiya ehpeiçiya, qa Wakantanka wopida eciya, Dawid tawicaśta ohiyapi kin heon. Hehan Dawid heya; Absalom kośka qon ni he, eya. Tuka wahośi hdi qon, wancahna Dawid wokiciya- 
ka eśta, yuśinyaye kte cin itonpa, qa, Owodutaton kin he icunhan Joab u maśi ce, cya.

Unkan unma wahośi u qon he Dawid en hi qa, Oyate śicapi wan wicaśtayatapi kis hipi qon Wakantanka kakiświcaya ce, eya.

Unkan Dawid iś, Absalom kośka qon ni he, eya. Unkan wahośi hi qon ayupte ça, Absalom tokece cin, oyate kin tona wicaśtayatapi kis hipi kin owasin eśta iś iyececapi unkanś, eya. Unkan wicaśta kin he Absalom wanna ta e ke cin, Dawid okahnigaa. Hehan nina iyokiśice ça ceya ye ça iye ti kin mahen ceya, qa heya; Absalom, micinkśi ; Absalom, micinksi ; Absalom, micinkśi. Wakantanka, tokin micinkśi on mațen; Absalom, micinkśi, micinkśi.

Hehan Dawid taakicita kin hdipi. Qa wicastayatapi kin iyokiśica nahonpi ; unkan iś eya iyokiśicapi. Wicaśtayatapi yuhapi kin waśtekidapi kin heon hececapi.

Absalom ktepi śni kupi kinhan, wicaśtayatapi kin itkowicakipe ça iwicayuśkin kta tuka. Hecen iye ti kin mahen owanji yanke ça, ite aihdahpa ceya; Micinkśi, Absalom, micinkśi, micinkśi.

Dawid Absalom on iyokiśica iyececa. Ecin Absalom tanyan ța unkanś, hecen Dawid ake mahipiya kin ekta wanhdake kta tuka. Wicacinca wanji ohan śice ça hecen hduśnapi kin he tehike. Absalom ohan śice ça kośka ecehnana tohan 
kin on Wakantanka teya. Tuwe hunkake ohokida śni eca he Wakantanka sicedake.

Hokśipidan, wicinyanpidan, Absalom śice ça on token te cin he nayalionpi. Tokin niyepi kin wanjidan Absalom iyenicecapi śnin. Tuka iye Wakantanka he niyuwaśtepi kte. Hecen nahanliin homakśiyopa ce ecin śni, wanna Wakantanka cekiya po; kinhan nanihonpi kta. Taku on he nanihonpi kta he. Iye Cinhintku țe cin heon.

\section{WOONSPE 18.}

DAWID, QA IYAYE CIN.

Absalom wanna ța, hecen Dawid ake Jerusalem ekta hde kta. Hecen ake oyate kin iyuhpa Dawid wicaśtayatapi kta cinpi. Hecen Dawid taoyate om Jerusalem ekta hda. Unkan Jordan wakpa kin akasam kihde cin hehan, wicaśta wan Dawid en hi, qa itokam makata elipeiçiye ça kicicajuju si. Unkan wicaśta kin he tuwe he. Wicaśta sica wan Dawid iyokiśica, unkan icunhan yaśice ça inyan on kinin qon hee.

Unkan Dawid kicicajuju he. Han, kicicajuju. Wicaśta qon he Dawid okilian qon heon iyokiśica ee śni; tuka Dawid ake wicaśtayatapi kin heon nakaha wikopa on hecon. Dawid iye kin kicica- 
juju, tuka Wakantanka e wicaśta śice cin he kicicajuju śni; ecin iyopeiciya wicake śni kin heon. Iho, hecen unkiś eya tuwe sicaya ecaunkiconpi eśta itkom unkicicajujapi kta. Ecin Wakantanka hecen eya, Niyepi kin ecanonpi śni kinhan, miśs eya cicicajujupi kte śni ce, eya.

Dawid Jordan wakpa akasanpa hde śni itokam wicaśta wan tokeca nakun wanyag hi. Wicaśta wijica qeya, Dawid iyokiśica un kin icunhan onśidapi qon he wanji ee. Unkan Dawid wicahinca qon he wopida eciye ca hehan Jerusalem ekta hde ape. Tuka wicaśta qon iye kin wanna wicahinca, heon cinca wan e Dawid ahda cin. Hecen Dawid he kici hda; qa Dawid wicalinea qon iiputake ça yawaśte.

Hehan Dawid Jerusalem ekta ki kin nina iyuśkin. Ake Koka kin he ikiyedan Wakantanka nina cekiyapi cin kin heon, qa oyate kin Wakantanka yatanpi qa idowanpi nalion kte cin hena on iyuśkin. Dawid Jerusalem etanhan tokan iyaye ciqon, he ehan ceye ça comnihdazi kin ota. Wośnapi Wokeya kin en Itancan Koka tawa kin yanke cin he itehanyan un kin heon hececa. Dawid Wakantanka waśtedake linca.

Wakantanka Dawid onśida, qa token wahoye cin he nayalionpi qon. Cinca kin wanji waśte kta, qa he Dawid țe cinhan he wicaśtayatapi qa nakun tipi wakan kağe kta, Wakantanka iwahoye ciqon. 


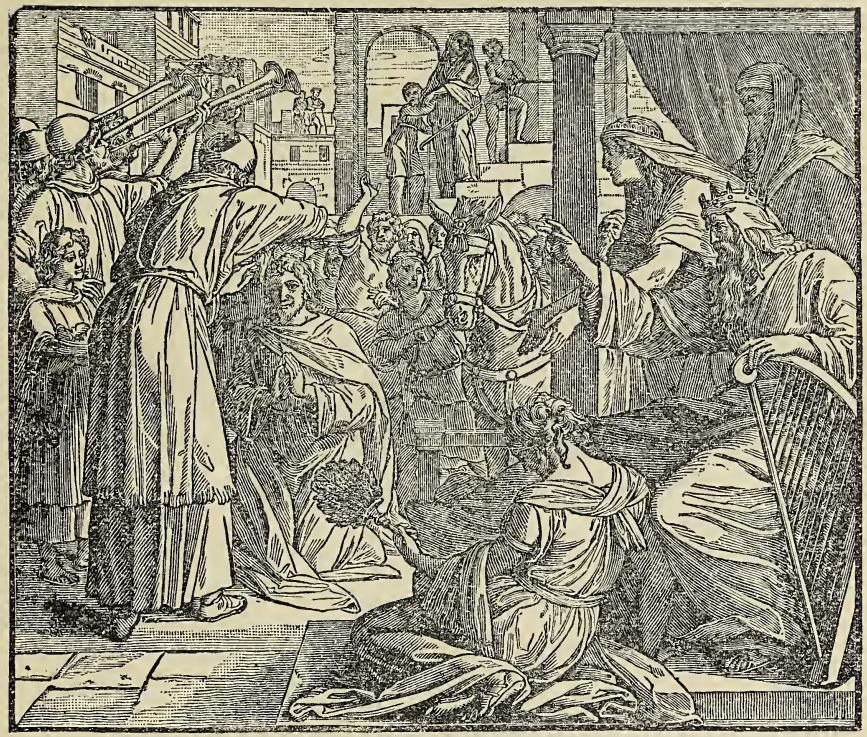

SOLOMON WICÁSTAYATAPI KAǴAPI.

Dawid cinca wan waśte kin Solomon eciyapi. Unkan Dawid wanna wicahinca, qa wanna kohanna țe kte cin heon, te śni itokam Solomon wicaśtayatapi kağapi cin. Hecen wośnapi itancan kin ihepi on Solomon pa sdakiya.

Dawid te śni itokam taoyate kin owasin witaya wicakico qa owicakiya cin. Qa wanna owasin ahi, hehan Dawid najinhan inajin qa heya; Eya Wakantanka tipi wan wecağa wacin, tuka iye Takantanka iyowinmakiye śni; okicizeta wicaśta ota wewaye cin heon etanhan. Qa micinca kin 
dee iye tipi wakan kagge kta. Wakantanka keya ce, eya.

Hehan Dawid Solomon heciya ; Micinkśi Solomon, Wakantanka anaggoptan wo, kinhan niyawaśte kta ce. Mehan Dawid on tipi wakan kagge kta wiyeya hnake cin hena Solomon kipazo; mazaska, mazaskazi, maza, inyan qa can. Qa tipi wakan kagapi kte cin on oyate kin iś taku mnakiyapi kta cinpi hecinhan, he Dawid iwicawanga.

Unkan oyate kin taku ota qupi, mazaska, qa inyan teliika, mazaskazi, maza, mazazi ko. Tipi wakan kağapi kte cin on oyate kin taku mnakiyapi kta iyokipipi kin Dawid wanyake cin nina iyuśkin ; oyate kin Wakantanka waśtedaka olianyanpi kin heon.

Iho, niś eya Wakantanka waśteyadakapi kinhan, taku duhapi kin he Wakantanka en takudan teyahindapi kte śni.

Hehan Dawid Wakantanka cekiye ça wopida eciya, Solomon tipi wakan kag̉e kta e iyowinkiye ça, oyate kin nakun wamnakiyapi kin hena on; qa nakun Solomon Wakantanka waśtedake ça oie kin anaggoptan kta cin kin he icekiya.

Hehan Dawid Wakantanka wakiyuśna. Hehan oyate kin owasin tiyata akiyahda. Unkan hehan iecadan Dawid ța.

Unkan Dawid naği kin toki un he. Wakantanka waśtedake ciqon wanna kici un. Maka akan 
SOLONON, QA TAKU WAŚTE KAHNIĠE CIN. 85

ni un qehan dowan wayapika, tuka mahpiya kin ekta iyotan sanpa ho waśte.

Dawid maka akan ni qehan hecen icaśkiyapi, Dowan wayapika, eciyapi.

Hokśipidan, wicinyanpidan, Dawid etanhan taku nayahonpi kin he onahon waśte yadakapi nace. Hokśidan ehantanhan Wakantanka waśtedake ça, taliinca wanunyanpi awanyake cin heca, qa he icunhan nina iyuśkin. Iho, hecen Wakantanka waśtedakapi kin heceedan on wicamdeze ça wiciyuśkin e nayahonpi. Hecen niś honikśiyopapi kin hetanhan wanna Wakantanka waśtedaka po, qa cekiya po; kinhan niyuwaśtepi kta. Dawid etanhan henana omdake kta.

\section{WOONSPE 19.}

SOLOMON, QA TAKU WAŚTE KAḦNIG்E CIN.

Solomon wicaśtayatapi kin, hehan taku tokaheya econ kin Wakantanka wakiyuśna.

Zion Paha kin en wośnapi ece kin hen econ śni; tuka Gibeon ekta wahna wośnapi mazazi wan tanka Moses kag̉e ciqon heciya i, qa hen taku ota Wakantanka wakiyuśna. Wakantanka wastedake cin he ikpazo, qa oie kin anagoptan cin.

Unkan hanyen Solomon iśtinbe cin hehan Wakantanka okíya, qa heya, Taku e cic̣u kta he, eciya. 
Unkan Solomon wicaśtayatapi, tuka taku waśte econ kta keś onspe kte cin tehika e sdonkiya; qa tuwe tohan taku sica ecakiciconpi eca Solomon en wohdag hipi keś, hecen tuwe śicaya econ kin iyeye ça on yaco kte cin nakun telika.

Unkan. Solomon heya; Oyate tanka chna wicaśtayatapi mayakağa; tuka nahanliin homakśidan; hecen taku tanyan ecamon onmaspe śni, heon ksapa makagga ye, hecen oyate kin en tanyan wamdaco kta, eya.

Unkan Wakantanka he nina iyokipi, qa heciya; Wowijice mayakida śni, qa tehan yani, qa tokaniyanpi kin ohiwicayaye kta nakun mayakida śni ; tuka wicoksape ecedan mayakida kin he iyomakipi: heon ksapa cicaġe ça wicaśta kin tuwedan yaksape cin he iyecece kte śni ce: qa nakun wowijice ciçu kta; hecen wicaśtayatapi hiyeye cin niśnana winijica, qa nitanka kta ce. Qa waśtemayadake ça mioie kin tanyan anayaggoptan kinhan, nakun tehan yani kta ce, eciya.

Hehan Solomon kikta, qa Wakantanka taku iwahoye cin he awakicin kin on nina iyuśkin. Qa hehan Jerusalem ekta ki, qa Zion Paha kin en Wakantanka Koka tawa yanke cin en taku ota wośna.

Wakantanka. Solomon ksapa kag̉e kta, keciye ciqon taku wanji wanna econ kta e hiyahde heciyatanhan ksape cin tanin kta. 
SOLOMON, QA TAKU WAŚTE KAḢNIĠE CIN. 87

Winohinca nom wakinicapi, qa Solomon en hipi, qa napin itokam inajinpi. Unma hokśsyopa wan ța e adoksohan, qa unma e hokśiyopa wan niyake yuha. Hokśiyopa napin cikcistinpidan, anpetu tonana wicatonpi. Hecen unma ni qon he nahanhin iyecinka yanke śni, qa nakun nahanliin tanyan wawanyake śni.

Unkan winohinca unma hokśiyopa wan ta $e$ adoksohe ciqon he iyokiśica itohnake, unkan hee heya; Hokśiyopa țe cin de mitawa śni, tuka unma ni kin he mitawa kin ee ce. Winohinca kin de kiciśnana tipi wanjidan untipi; unkan hanyetu wan en iye cinca kin kpața; unkan hecen miśtinma icunhan, micinca qon imakicu, qa țe cin de en ahionpa. Hecen hanhanna wekta, unkan te cin de yuha munka, tuka wancahna mitawa śni kin sdonwakiya ce, eya.

Tuka unma qon iś heya; Iewicaka wodake śni ce, țe cin he nitawa, qa miś ni kin de mitawa ce, eya. Tuka ake unma qon iś, Hiya, țe cin de nitawa kin ee, qa ni kin he miś mitawa ce, eya.

Unkan Solomon token on unma tukte wicakapi kin iyeye ça, cinca kin kicu kta he.

Wakantanka Solomon ksapa kaġe cin heon token iyeye kte cin awacin.

Unkan Solomon taokiye kin wanji kipan, qa mazasagye wan auśi, qa heciya; Hokśiyopa wan ni kin he cokaya kicakse ça, winohinca kin kinu- 
kan wicaqu wo; napin he tawapi keyapi ce; hecen kiuukan yuhapi kta ce, eya.

Tuka winohinca qon he śicahowaye ca, Kte śni ye, tuka iye eśta qu ye, niyake e yuhe kta

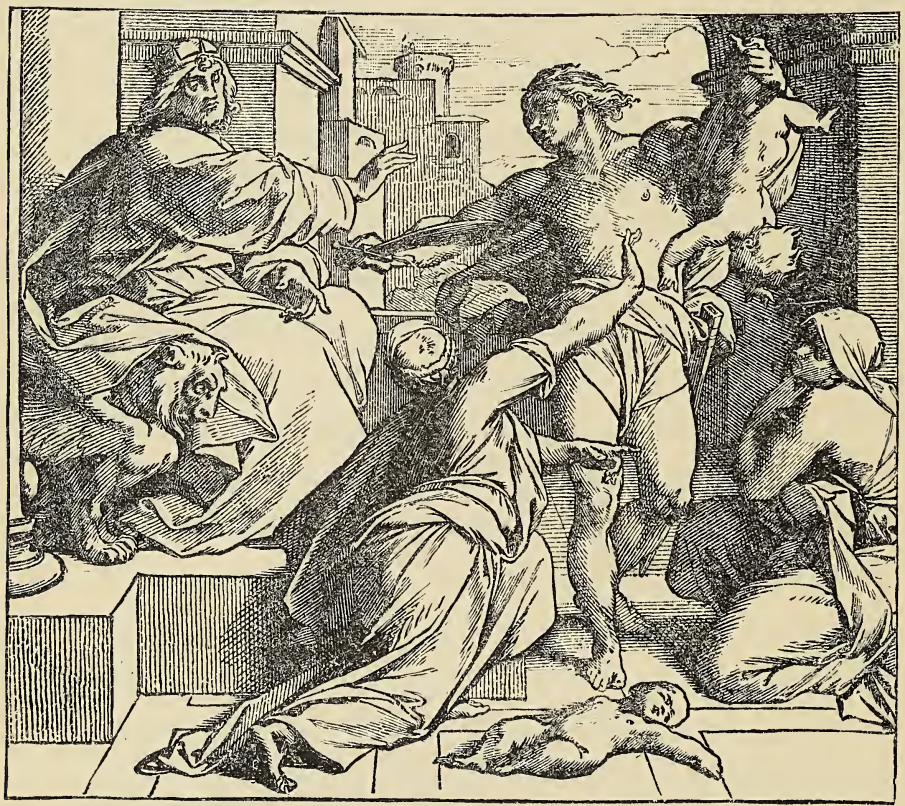

SOLOMON KSAMYAHAN WAYACO.

ce, eya. Tuka unma qon iś, cokaya kicaksa ye, hecen kinukan unhapi kta ce, eya.

Iho, hecen unma tukte hunku kin ee he. Unma tehinda kin hee.

Hecen Solomon sdonye ça taokiye kin heciya; 
SOLOMON, QA TAKU WAŚTE KAH'NIǴE CIN. 89

Unma tehinda kin he hunku ee, kicu wo, hduhe kta ce, eya.

Solomon hokśiyopa wan kicakse wicaśi qon he awicake śni, tuka heciyatanhan on unma tukte tawapi kin iyeye kta nakaeś hecon, qa hecen sdonye. Tho, hecen Solomon ksamyahan econ yadakapi nace. Ecin Wakantanka wicoksape iwahoye ciqon heon hececa.

Unkan Israel oyate kin Solomon taku econ kin he nalionpi, unkan on ksapedakapi qa tonhanpika, Wakantanka he ksapa kaġe ça, heon hecen econ kin sdonyapi.

Hokśipidan, wicinyanpidan, niś yaksapapi yacinpi kinhan, nina wowapi yawapi, qa waonspeic̣iciya po; heciyatanhan on taku sdonyayapi kta ce.

Unkan Wakantanka Solomon wicoksape qu qehan, Yaksape cin tuwedan iyenicece kte śni ce, eciya. Tuka he iyohakam Jesus maka akan hi kin, hehan Solomon wicoksape tawa qon he wanji isanpa e iwahounyanpi, iye Taniya Wakan kin hee. Iho, hecen nina yakidapi kinhan nicantepi kin ekta ehnake ça, hecen taku śice cin śicedake niyanpi, qa taku waśte kị he en aniyanpi kta. 


\section{WOONSPE 20.}

\section{SOLOMON, QA TIPI WAKAN KIN.}

Wakantanka Solomon Tipi Wakan wan kağe kta keciya e nayahonpi qon.

Solomon tipi kin he taku ota on kahwicakiya; mazaska, qa mazaskazi, qa maza, qa mazazi, qa inyan, qa can. Qa ticag̉api kin iś nakun wicotapi. Unkan tipi kin he token kaġapi kte cin Wakantanka Dawid ecen okiyaka; hecen Dawid iś ecen owa ehnaka.

Unkan Solomon tipi kin he Zion Paha kin akan kağe sni, tuka icunonpa paha wan tanka akan kaliwicakiya.

Qa Solomon tipi ahdehe cin inyan tankinkinyan ece on kaliwicakiya. Qa hanteśadan can ece on akantanhan kağapi ; qa timahentanhan owancaya mazaskazi ipawintapi; hecen timahentu kin wiyakpa kin he oiyokipi.

Qa mazaskazi peta ijanjan ihupa wikcemna en hdepi. Qa mazaskazi wahna wotapi wan akan ag̀uyapi hnakapi ; qa ahna waazintonpi kin nakun mazaskazi. Qa tipi wakan kin ihdukśan hocoka wan kaliwicakiya. Qa hehan itankantanhan kin inyan on acankaśkapi. Qa hocoka kin mahen nape ohdujajapi mazazi wikcemna hdepi; hena ohna woteca wawicayuśnapi kta eca, itokam on 
wicayujajapi ece. Qa nape oyujajapi kin wanjidan iyotan tanka kagge ça, hehan mazazi tatanka akenom kagie ça, hena witaya ewicahdepi, qa cankahupi kin akan nape oyujaja tanka qon he ehnakapi ; qa mini ojudan okaśtanpi, hecen wośnapi itancanpi kin hetanhan mini on ihdujajapi kte cin icupi ece.

Unkan Solomon hocoka kin en wahna wośnapi wan tanka linca e ehde; hecen woteca wawicayuśnapi ece e kta.

Unkan tipi wakan kin tanyan yuśtanpi kin, hehan maka akan tipi kagapi kin heceedan iyotan waśte. Hehan Solomon oyate kin owasin tipi wakan kin en upi cin. Qa wośnapi itancanpi kin iś Koka qon he en ayapi; tipi wakan kin mahen ehnakapi kte cin he en ozantonpi, qa tiyopa tonyan hdepi. Ozanpi kin he waśte hinca, qa can nom mahpiya ohnihde wicakagapi, qa akapatanhan kin owancaya mazaskazi iwicapawintapi, qa Koka qon akan ewicahdepi. Qa nakun nom iyotan tankinkinyan wicakagapi qa hupahu hdumdahkiya en wicahdepi, qa he en owanka wakan eciyapi.

Qa he icunonpatanhan iś dowanpi kin yakonpi, wośnapi itancanpi koya owasin wokoyake ska ece koyakapi, qa candowankiyapi ocaje ota on dowanpi, qa wośnapi itancanpi kin apa iś cotanka unpi, qa odowan kin en kaken eyapi; Itancan wopida 
qu po, iye waśte ce, towaonśida kin owihanke wanica ce.

Wakantanka idowanpi qa yatanpi kin he icunhan Wakantanka towitan kin tipi wakan qon ojudan hi. Unkan wośnapi itancanpi qa dowanpi qon owasin tin yukanpi okihipi śni, qa tankan hocoka kin ekta inajinpi.

Solomon he wanyake cin nina iyuśkin; Wakantanka tipi kicage cin he iyokipi, qa heon towitan en tanin kin heon.

Hocoka kin en mazazi oyanke wan tehanwankantu Solomon kaliwicakiya, qa iśs he akan najin, wahna wośnapi wan tanka kaggapi qon he icahda. Unkan Solomon hen akan isto akaġankiya canpeśka makehde inajin, qa Wakantanka cekiya. Tawocekiye kin hanske liinca, tuka etanhan ptecedan nayahonpi kta. Oyate kin tona woahtani on iyokiśicapi kin hena Wakantanka wicakicicajuju kta he icekiya.

Unkan Solomon wocekiye kin ihunnikiya, hehan mahpiya kin eciyatanhan peta wan hiyu, qa wahna wośnapi kin akan woteca wawicayuśnapi kta wicahnakapi qon owasin hulinahwicaya. Tuka oyate kin eqe tuwedan kiunniwicaye śni, woteca kin hena hulinaliwicaya. Wakantanka hee hecon. Oyate kin cekiyapi qa wakiyuśnapi kte cin he iyokipi kin heon hecon.

Unkan Wakantanka taku taninyan econ kin 
SOLOMON, QA TIPI WAKAN KIN.

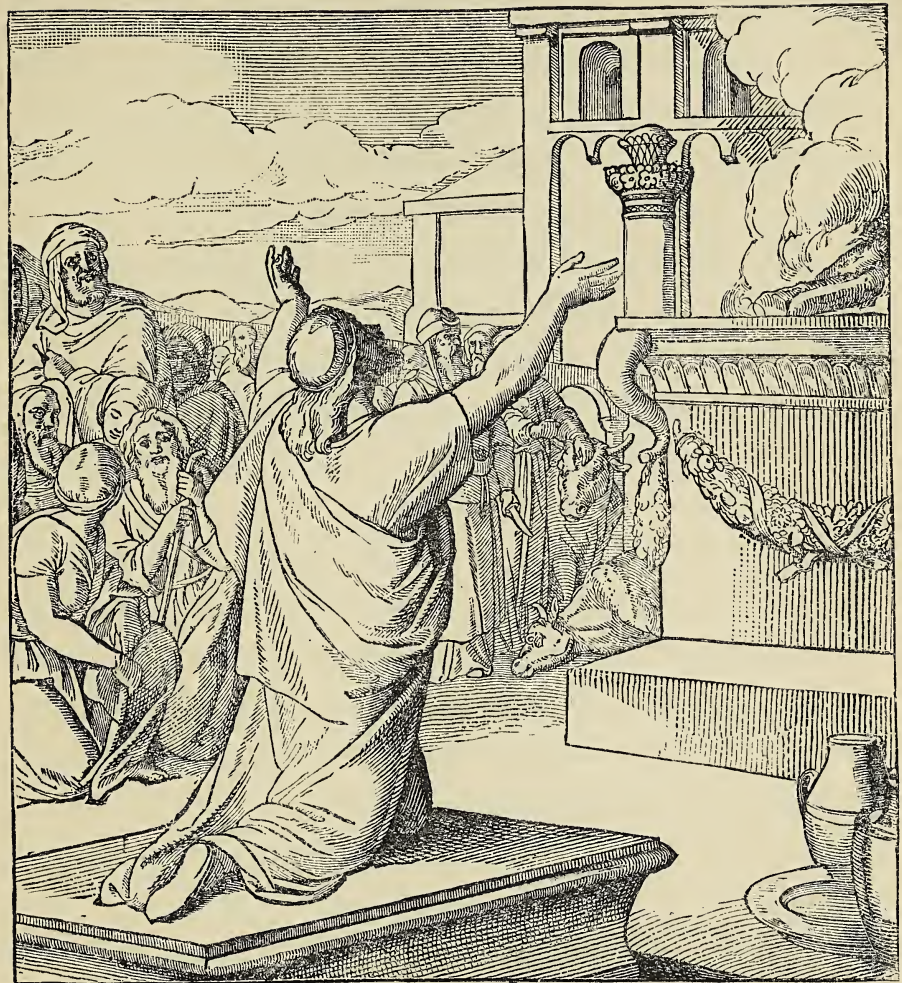

SOLOMON TIPI WAKAN YUŚTAN QA CEKIYA.

he oyate kin wanyakapi, unkan owasin makata elipeiçiyapi, qa Wakantanka yatanpi, qa heyapi; Iye waśte, qa towaonśida owihanke wanica ce, eyapi.

- Hehan oyate kin owasin tiyata akiyahda, tuka tipi wakan kin en wośna ahi, qa Wakantanka 
cekiyapi ece. Qa tipi wakan kin en anpetu hanyetu koya, ohinniyan candowankiyapi on Psalm odowan kin hena ahiyayapi. Hecen tona en ikiyedan unpi kin ohinni nation unpi ; hecen tipi kin hen wiciyuśkin qa oiyokipi linca.

He oiyokipi kin isanpa owaśtecaka wan ekta Wakantanka yanka; qa towitan kin anpetu wi kin isanpa iyoyanpa; qa he ohinniyan qa tipi wakan wan en wiciyuśkin nayahonpi qon he isanpa heci wiciyuśkin.

Hokśipidan, wicinyanpidan, heciya waun qeś ecannipi he. Taku nom Wakantanka kidapo; wayahtanipi kin he nicicajujupi kta icekiya po, qa Woniya Wakan niçupi kta icekiya po, hena iwahounyanpi ce. Unkan taku on etanhan hena iwahounyanpi he. Jesus cansusbeca akan te cin heciyatanhan on hecen wahounyanpi.

\section{WOONSPE 21.}

SOLOMON, QA WICAŚTAYATAPI WINOḦINCA KIN.

Unkan ake Wakantanka hanyetu wan en Solomon okiye ça heciya; Tipi wakan kin ohna wocekiye emayakiye ciqon he nawalion ce. Anamayağoptan kinhan, hecen ciyawaśte kta ce; tuka anamayaggoptan śni, qa taku sica ecanon kinhan, 
sinwahda kta ce; qa tipi wan waśte yakag̉e cin he jujuwahe kta ce, eciya.

Solomon ksape ça nakun wijice hinca Wakantanka hecen iwahoye cin iyecetu. Wita wata ota kagga, qa nakun otonwe ota kaġa; qa tipi iyotan waśte wan kağe ça oyanke wan tanka en kağa; qa owancaya mazaskazi akalipapi, qa en amaniya ece kin śakpe owecinhan, qa hena otoiyohi mnaja owicakağapi kin heca, śakpekpe anokatanhan yukanpi, qa oyankeye cin itananokatanhan nakun nom yukanpi.

Solomon ksape ça taku eya eca ksamyahan eye cin, heon oyate kin makoce tehantanhan keś, heciyatanhan ie nahion hipi, qa hena taku kahipi ece, mazaskazi wiyatke, qa mazaska, qa wokoyake, qa śuktanka, qa śonśonna ko kahipi ece, hena itu qupi.

Hecen Solomon sanpa wijica icağa. Qa wita wata kin hena iś miniwanca akasam yewicaśi, qa heciyatanhan mazaskazi, qa mazaska, qa zitkadan ocaje waśte ko ota kahdipi ece. Hecen wicaśtayatapi maka ohnaka kin Solomon ecedan wijica.

Solomon taku ota sdonya. Can ocaje kin owasin sdonye ça woteca, qa zitkadan, hoğan qa wamduśkadan, qa taku cikcistinna kinyan unpi kin owasin caje sdonwicaya. Tuka taku wanji e iyotan waśte sdonya, Wakantanka hee token iyokipi- 
ye kte cin he sdonya, qa hecen oyate kin ksamyahan wahokonwicakiya.

Unkan wicaśtayatapi winohinca wan makoce tehan ti, tuka Solomon ksape cin he nahon; unkan ia nalion kte hinca, qa ti kin nakun wanyaka cin.

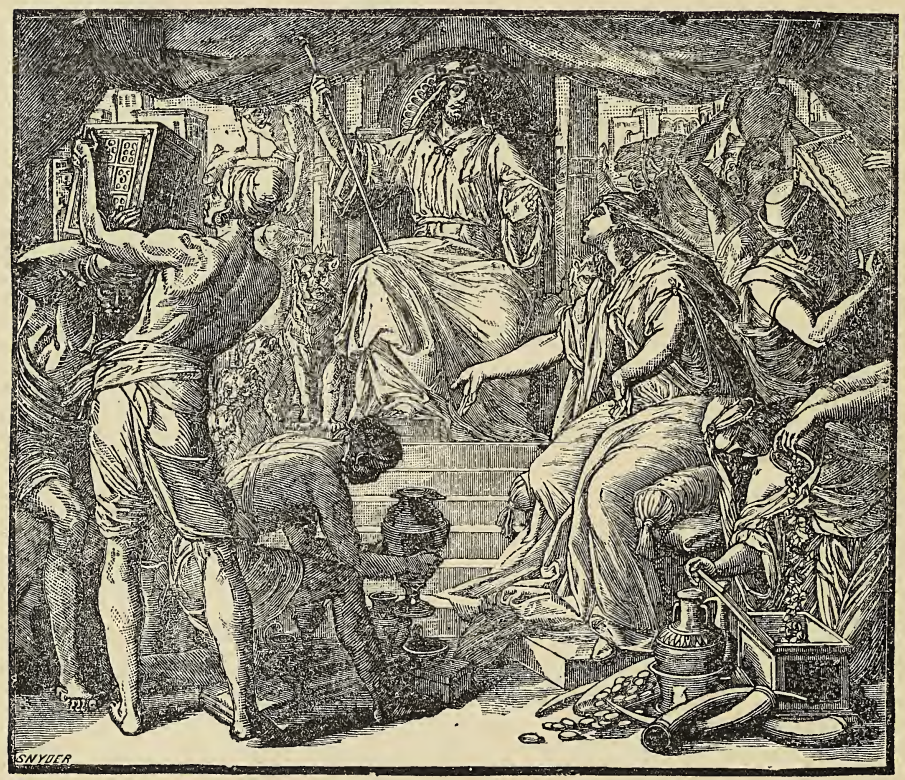

ŚEBA WICAŚTAYATAPI WINOḢINCA KIN.

Winohinca kin he Śeba wicaśtayatapi winohinca eciyapi. Iśs eya wijica. Hecen taokiye ota om u, qa mazaskazi qa inyan tehika ko ota Solomon kai qa taku ota iwanğa. Okinni Wakantanka 
on etanhan taku iwanga nace. Iye tamakoce ekta Wakantanka oie nalion śni un, hecen on nalion cin kta iyececa. Hecen Solomon iś eya tanyan kuwa, qa taku iwange cin owasin okiyaka; qa tipi kağe cin hena nakun wanyagkiya. Unkan hena nahon qa wanyake cin, hehan heya; Yaksapa nacilion qon ehanqon wicakapi hinca ce ; nitaokiye nikiyedan najinpi kin ohinni ieksapa iyae cin de nahonpi kin toke hin iyuśkinpi cen eca, Nitawakantanka wicaśtayatapi nicağe cin he yawaśtepi ce, eya.

Hehan mazaskazi inyan telika ko ahi qon hena Solomon qu. Hecen iś taku cin kin owasin Solomon qu; qa hena yuha iye tamakoce kin ekta kihda.

Unkan Śeba wicaśtayatapi winohinca kin wicoksape nahon kin hena awacin iye ti kin ekta ki. Hecen wicoksape nalion kin he taku ikce qupi kin he isanpa. Hena kiksuye cin on wakagapi cekiye cin he ehpeye ça, Wakantanka e waśtedaka iyececa.

Hokśipidan, wicinyanpidan, hena dawapi, qa he icunhan, Śeba wicaśtayatapi winohinca kin wanniyakapi ehan nina widuśkinpi nidakapi naceca.

Tuka Jesus iyotan wicoksape ota eya. Solomon isanpa ksapa; qa oie kin hena wanna wowapi wakan en dawapi. He nina onspeniçiciyapi kinhan on iduśkinpi kte cin hee. 


\section{WOONSPE 22.}

SOLOMON, QA WAKA $\dot{G A P I}$ KIN.

Wakantanka Solomon nonpa akihde okiya e nayahonpi qon. Tokaheya kin he wicoksape iwahoya; qa inonpa kin he iye oie kin anagioptan kinhan yawaśte kta keciya. Unkan Solomon Wakantanka anagoptan he.

Anag̉optan, tuka wanna wicaśta tanka kecin hehan anag̉optan śni, qa sicaya econ. Tawicu ota qa heon woahtani tanka econ. Hehan nakun Jakob tawicu nonpa e nayahonpi qon. Tuka he wanakaja qa he ehan heconpi. Tuka otokahe ehantanhan wicaśta itoiyohi tawicu wanjidan kte cin he Wakantanka waśtedake. Qa dehan wicoicagge kin tawicu wicanonpa kin he Wakantanka sicedaka e sdonunyanpi.

Unkan Solomon tawicu opawinge śakowinpi. Unkan he tokeca e henake seh wicayuza he. Solomon ksape ça wijica kin heon ocim itan, qa iśnana wicaśtayatapi tanka, qa on etanhan tawicu ota kin he itan cin, qa heon hecon.

Tawicu kin owasin śicapi qa wakagapi cekiyapi. Qa ecen unhanketa Solomon iś econkiyapi; qa hecen Jerusalem en paha wan tehanwankantu akan wahna wośnapi wan kaġapi kin he wicakicağa; qa iś iye hinca wakağapi cekiya. Hecen 
Solomon ake nakun sanpa hin sicaya econ. Tohinni taku hecehin śica econ kte śni kecannipi tuka nace. Hecen witkotkoya econ. Ecin, hena can qa inyan wakagiapi kaġapi, hecen hena taku śni, tuka cekiye.

Tona he wanyakapi kin tokeh oiyokiśicapi cen eca. Winohinca qeya wakaġapi wakiyuśnapi, qa

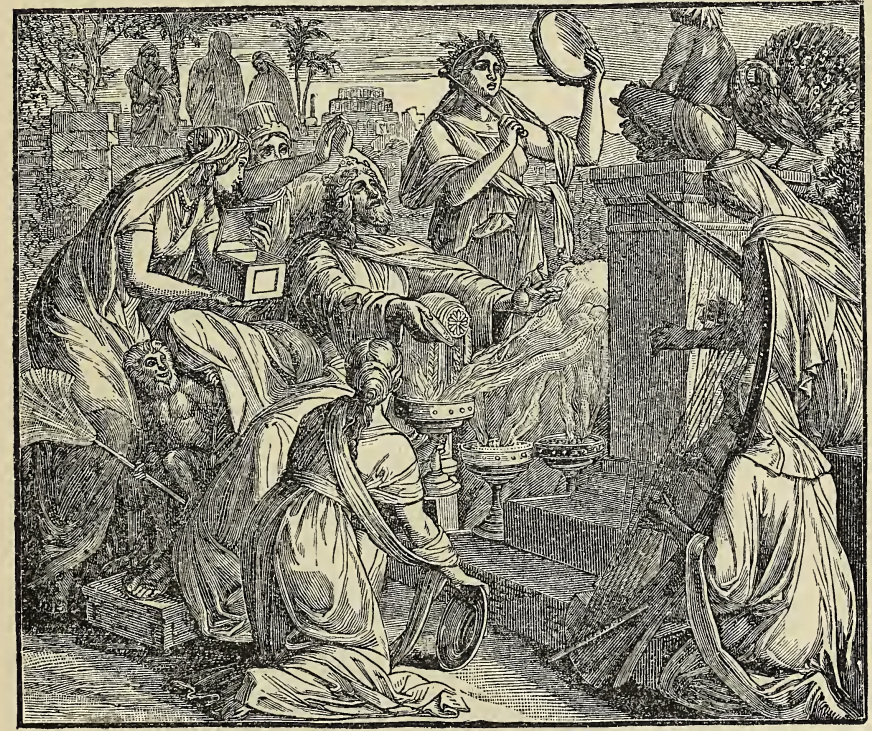

SOLOMON WAKAG்API WAKIYUŚNA.

wizinye ko izinyapi kin en, icunhan Solomon iye hinca wakağapi kin itokam makata ehpeiçiye cin he.

Hecen Wakantanka he sinhda qa Solomon heciya; De ecanon kin heon etanhan nitaokiye kin 
wanji iye wicaśtayatapi ic̣icagge kta ce ; qa nicinca wan wicaśtayatapi kte cin he makoce tankaya ki kta ce; tokeh nițe ça hecehnana. Niye ni yaun kin den wicaśtayatapi kin he ecicihdaku śni; tuka he niyate Dawid on etanhan he ecamon śni ce, eciya.

Solomon te sni itokam wahtani kin de on iyopeic̣iya nacece; tuka hecon kin tanyan sdonwaye sni. Iyopeiçiya iyececa. Ecin iyopeiçiye śni ehantanhan Wakantanka teliiya kakiśsye kta sdonkiya.

Hecen Solomon te cin hehan cinhintku wan wicaśtayatapi ; tuka ecahankeya iś Solomon taokiye wanji iye wicaśtayatapi ic̣icağa; ookiye kin he Jeroboam eciyapi. Hecen Jeruboam Kanan makoce kin tankaya icu, qa Solomon cinhintku kin e cistiyedan yuha.

Wakantanka Solomon hecen eciye ciqon iyecetu. Oyate kin tona Wakantanka anagoptanpi śni eca kakiświcaya ece.

Hoksipidan, wicinyanpidan tokin Solomon iyecen inicaġapi snin. Solomon cistinna etanhan Wakantanka waśtedaka, unkan hehan wicaśta tanka ehan i, unkan ayuśtan kin he. Tuka niśs nicistinpidan kin detanhan Wakantanka waśtedaka po; hecen iye tohanyan cin kinhan hehanyan yanipi kta. 


\section{WOONSPE 23.}

JEROBOAM, QA NAPE ŚNIJE QON.

Jeroboam Kanan makoce kin en ohna tankaya wicaśtayatapi ic̣icagge cin he nayahonpi. Tuka Jerusalem otonwe kin Jeroboam makoce tawa kin ohna śni; tuka Solomon cinhintku tawa kin hee ohna. Hecen Solomon cinhintku kin he tawa kin heon waśte. Ecin Jerusalem en tipi wakan he cin heon etanhan.

Kanan makoce en oyate ohnaka kin owasin Jerusalem en tipi wakan he cin he ohna Wakantanka ohoda ahi kta e Wakantanka econ wicaśi. Hecen Jeroboam is eya en Wakantanka ohoda $u$ kta iyececa; tuka hecon kta cin śni; wicaśta śice cin heon etanhan; qa iye taoyate kin nakun heci yapi kte cin iyowicaki śni.

Jerusalem en wicaśtayatapi tokeca yanke cin heon, okinni iye taoyate kin heci ipi kinhan, wicastayatapi unma kin e waśtedakapi kta ikope cin, heon heci yapi cin śni, qa sanpa hin śicaya econ. Mazaskazi ptejicadan wakağapi nom wicakag̉e ça, iye tamakoce kin ahankeya otonwe kinukan en ewicahde, qa hecen iye taoyate kin he ohoda wicaśi. Qa heya; Jerusalem ekta dapi kte cin otehan yanka ce; hecen wakagapi kin dena e ohowicada po, ewicakiya. 
Tuwe iye kin śica keś nakun sanpa wicaśta tokeca ekta sicaya econwicaye cin he iyotan sica. Hokśipidan, wicinyanpidan, he kiksuya po. Yaśkatapi kin en icunhan, ihnuhan taku śica econki-

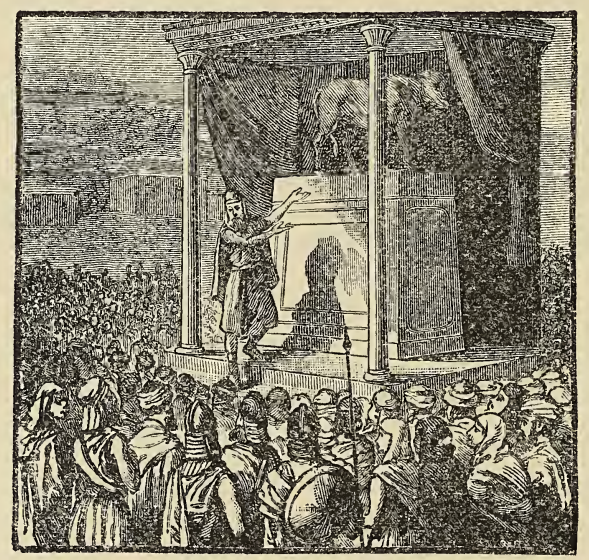

MAZASKAZI PTEJICADAN WAKAĠAPI KIN.

ciciyapi śni po. Hecanonpi kinhan wakanśsica iyenicecapi kta. Wakanśica ohinniyan wawiyutanye ça oyate kin wahtaniwicaya ece.

Jeroboam iye linca wakaġapi kin ohoda i; unkan he icunhan Wakantanka Waayata wan en ye sii, qa taku śica econ kin he okiciyag śi. Jeroboam wahna wośnapi kin icahda najin, qa wizinye izinya ican Waayate cin en i; qa oyate kin ptejicadan wakaġapi wan cekiyapi kin he Wakantanka sinhda, qa token econ kte cin okiciyaka qa heya; 
Wakantanka de śihda ce ; qa wahna wośnapi kin de kakse kta, qa cahota kin hena makata hinhpaye kta ce, eya.

Jeroboam he nakilion kin śihda, qa on Waayate cin kakiśya cin; qa isto akatinyan hduze ça, Yuza po taokiye kin ewicakiya. Jeroboam isto akatinyan hduze ciqon ecehnana Wakantanka isto qon patinyan, qa hecen tokadan hdukśije śni. Qa he icunhan iś wahna wośnapi qon kakse, cahota qon owasin makata hinhpaya, Waayate cin heye ciqon iyecen.

Hehan Jeroboam nina yuśinyaya; qa isto kin tuwedan asnikiciciya okihi śni, tuka Wakantanka ecedan he okihi kin sdonkiya. Hecen Waayate cin heciya; Nitawakantanka kin cekiya ye, isto kin asnimaye kta, eya.

Unkan sicaya ecakicon wacin qon iye qe wocekiye ekiciya he. Han, iye qe wocekiye ekiciya, qa hecen Wakantanka Jeroboam isto kin asniyan.

Hehan Jeroboam icimana wayyate cin kiunniyan cin śni, wikope cin heon etanhan. Tuka wakag̉api ohoda kin hee ayuśtan śni. Nakun iyowinye śni econ, qa iye taoyate kin iś nakun wakagapi cekiyewicakiya. Hecen Jeroboam sice cin he Wakantanka śihda

He tokeca Jeroboam Wakantanka kokipe śni he; isto kin patinye ciqon he wanhdaka tuka. 
Wakantanka Jeroboam țeye ça wakanśica ti en iyohpeya okihi.

Hokśipidan, wicinyanpidan, Wakantanka taku sihda unyanpi kte cin he wowitonpeke. Niś eya ninapepi kin on taku sica ecanonpi kinhan, icunhan Wakantanka ninapepi kin kiunniyan okihi. Iśnana waonśida hinca. Tuka anaungoptanpi śni kinhan unnagipi kin peta kasnipica śni ehpeunyanpi kta.

\section{WOONSPE 24.}

ELIJAH, QA KANG்I KIN.

Jeroboam Israel en wicaśtayatapi, qa Solomon cinhintku kin iś Juda en wicaśtayatapi.

Jeroboam țe cin hehan ake wanji tokeca Israel en wicaśtayatapi kiyapi. Tuka ake te ça ake wanji tokeca. Hecen wicaśtayatapi ota owecinhan Israel en wicaśtayatapi unpi. Tuka owasin śicapi; qa Jeroboam ptejicadan wakagapi wan kage ciqon he owasin ohodapi. Wicaśtayatapi kin hena owasin caje owicamdaka eśta otapi; hecen okinni yeksuyapi kte śni; heon owicamdake śni.

Unkan hena owasin țapi, hehan ake wanji tokeca wicaśtayatapi, he Ahab eciyapi. Wicaśtayatapi kin owasin śicapi, tuka he iyotan sica. Qa ake nakun sanpa hin winohinca wan wakagapi ohoda, unkan he yuza. Winohinca kin he makoce 
tokanta wicaśtayatapi wan ateya. Winohinca kin he wakagiapi ohoda aicahyapi, nakaeś waśtedake linca, qa nakun taku ota sica econ. Winohinca kin he Jezebel eciyapi. Israel wicaśtayatapi winohinca kin eya cajeyatapi, Ahab hihnaye cin heon etanhan hecen eciyapi.

Jezebel wakaggapi iyotan tehinda kin he Baal eciyapi. Unkan hihnaku Ahab wakagapi kin he ohodakiya. Hecen Ahab ptejicadan wakagiapi wan ohokida qon he iyecen nakun Baal ohoda; qa iye totonwe kin en tipi wakan wan Baal kicaga, qa he en wicota waonspekiyapi oyate kin token Baal ohodapi kte cin he onspewicakiyapi. Wicaśta kin hena wakaġapi waawayata ewicakiyapi ; hecen Jezebel wicaśta kin hena cantewicakiye hinca. Qa nakun oyate kin tona wakagapi ohodapi kin hena nina cantewicakiya; qa tona e Wakantanka ohodapi sdonye cin hena wicakte cin.

Israel oyate kin he en wicota Wakantanka waśtedakapi. Hecen hena wakagiapi kin he ohodapi śni. Unkan hena Jezebel wicakte cin. Hecen imnija ohidoka ohna naiçihmanpi.

Unkan Wakantanka oyate kin hena nina waśtewicakidaka.

Unkan Israel makoce kin he en waayata wan waste e ti, Elijah eciyapi; hee oyate kin wakagapi ohodapi kin ayuśtanpi qa Wakantanka e ohodawicakiya cin., Unkan oyate kin taku akipapi 
kte cin he Wakantanka Elijah nina okiyaka. Hecen Elijah nina Wakantanka cekiya.

Ahab tawicu Jezebel kici Elijah śicedakapi, qa ktepi cinpi. Waśte kin heón etanhan. Qa Israel oyate kin wicota wakağapi cekiyapi. Elijah wanyake cin on nina icanśica, qa śicaya econpi kin heon iyopeiçiyapi qeś, ecin. Ecen unhanketa Wakantanka oyate kin kakiświcaya.

Wakantanka maǵaju kin he anapta. Hecen wi ota maggaju śni ; qa nakun cu śni. Hecen wato kin amaśte eceedan on owasin śnija; qa wojupi kin owasin nakun icağe śni; qa waskuyeca can aicag่e ciqon owasin nakun icagge śni. Hehan Israel oyate nina cante wicaśica. Tuka Wakantanka iś oyate kin taku sica econpi kin he iyopeiçiyapi cin.

Unkan Elijah iś he icunhan token un he.

Wakpadan wan onalibeyata wanka, unkan hè ekta Wakantanka Elijah yeśi. Ahab kte cin kin heon hen naiçiłman. Qa tokeśta woyute kaipi ce kta, Wakantanka keya wahoya.

Hecen Elijah wakpadan kin ekta i, qa he en mini yatkan. Qa hanhanna hitayetu owasin kanği qeya ag̉uyapi qa tado ko kayapi qa en kiyuśnapi ece. Hecen Elijah woyute qa mini ko icakije śni tanyan un, qa takudan sanpa cin śni.

- Unkan he tuwe kangi kin hena wahbadan waonsida wicakagia he. Wakantanka he hena 
ELIJAH, QA KANG்I KIN.

hecen econwicakiye. Taku kinye cin kangi kin he iyotan watoghda. Tuka Wakantanka ecedan taku owasin econ okihi. Elijah hena wokaupi

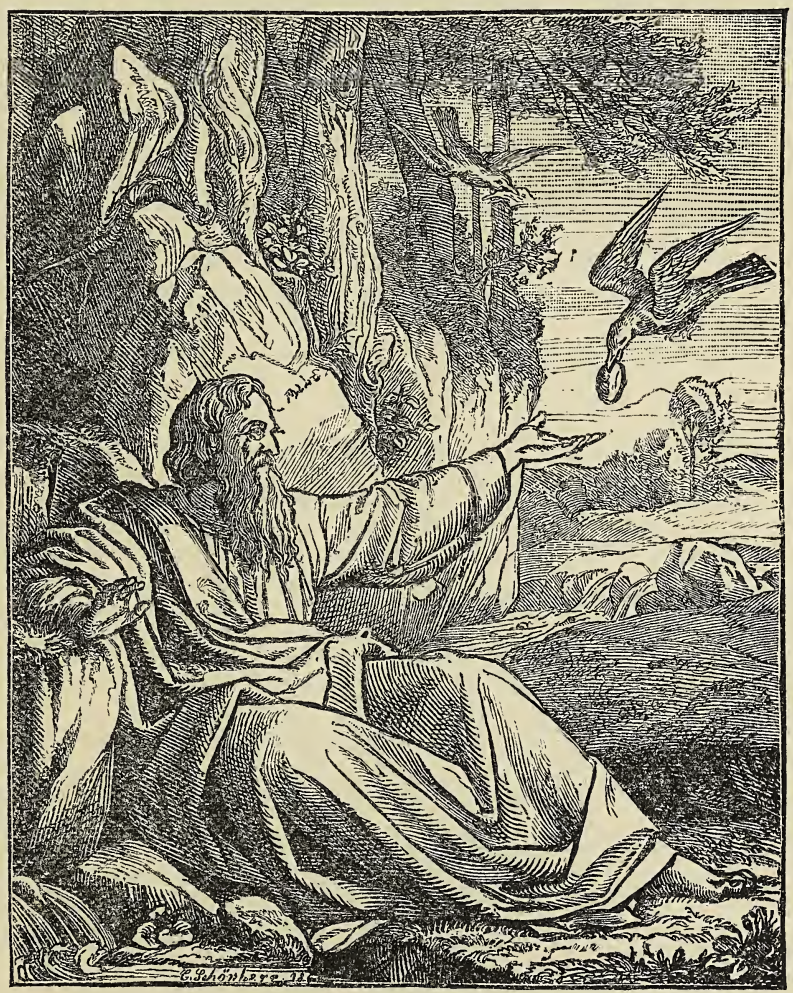

KANG்I KIN ELIJAH WO KAUPI.

wanhdaka eca on tokeh iyuśkin cen eca; qa on tokeh Wakantanka wopida eciye ca yatan cen eca. Wakantanka hecen wahounyanpi; Tuwe onśike 
ça wotektehda un kinhan cekiye ça kida kinhan qu kta keya.

Elijah wakpadan kin he en iśnana hin un. Tuka iye kin iśnana un kecanic̣in śni; Wakantanka he kici un e sdonkiya. Unkan ocim wakpadan qon oyahe aya. Tuka maġaju śni hecen ecen oyahe. Unkan wanna mini kin puza. Hecen hehan Elijah token un kta he. Wakantanka wakpadan kin he ake miniyan okihi; tuka he econ kte śni. Heon etanhan Wakantanka Elijah wakpadan kin he ayuśtan si, qa tokan yeśi. Elijah tokiya i kin he ake nayahonpi kta.

Hokśipidan, wicinyanpidan, Wakantanka Elijah tanyan kuwa kin he nayahonpi. Iho, niś Wakantanka waśteyadakapi, qa wacinyayapi kinhan tanyan niyuhapi kte.

\section{WOONSPE 25.}

ELIJAH, QA WIWAZICA KIN.

Elijah wakpadan wan en un, tuka wanna mini kin oyahe qon; hehan icunonpa itehanyan wiwazica wan ti. Unkan he ekta Wakantanka Elijah yeśi; kinhan wiwazica kin he woqu kta. Tona wiwazicapi kin hena onśikapi ece. Hecen iśs eya wiwazica kin he onsike hinca. Qa magaju śni kin hehantanhan oyate kin taku yutapi tonagnana hin tonpi; wojupi kin tanyan icage śni kin heon.

Hecen Elijah Wakantanka heciya yeśi qon, 
ekta ya. Kanan makoce kin opta ye.ça, inaśdoke cin hehan otonwe wan en i. Unkan oyate kin he Wakantanka oie sdonyapi śni; hecen wakağapi ecedan cekiyapi.

Unkan Elijah otonwe tiyopa kin en i; unkan inyun, winohinca wan onśika e cansakadan pahi e wanyaka. Unkan Elijah wiwazica wan ti ekta ye ciqon he hee sdonya. Hecen Elijah winohinca qon kipan qa heya; Ceciciya ce, mini onġe makau ye, mdatke kta ce, eya.

Elijah nina ipuza liinca. Ecin tehan mani ye ça nakun mini tonana ecen ye cin heon.

Unkan wiwazica kin waonśida, hecen Elijah mini qu. Hehan ake Elijah heya; Ceciciya ce, ag̉uyapi onśpa maqu ye, eciya.

Unkan wiwazica kin heya; Ag'uyapi takudan wahnake śni ce; qa ehake ag̉uyapi mdu nape ohnaka ecen koka kin ohna yanke cea, wihdi nakun tonana hin ojuha kin en un. Hecen cansaksakadan wapahi kin dena on cewati, qa ağuyapi zimzipedan wakağe ça, micinkśi kici wate kta ce: qa he temunkiyapi kinhan, hehan akihan unțapi kta ce, eya.

Unkan Elijah wiwazica woyute yuhe cin owasin temkiye kta he. Elijah token eye kte cin owasin Wakantanka he onspekiya.

Hecen Elijah wiwazica qon heciya; Miye tokaheya ağuyapi zimzipedan micaġa wo; qa hehan 


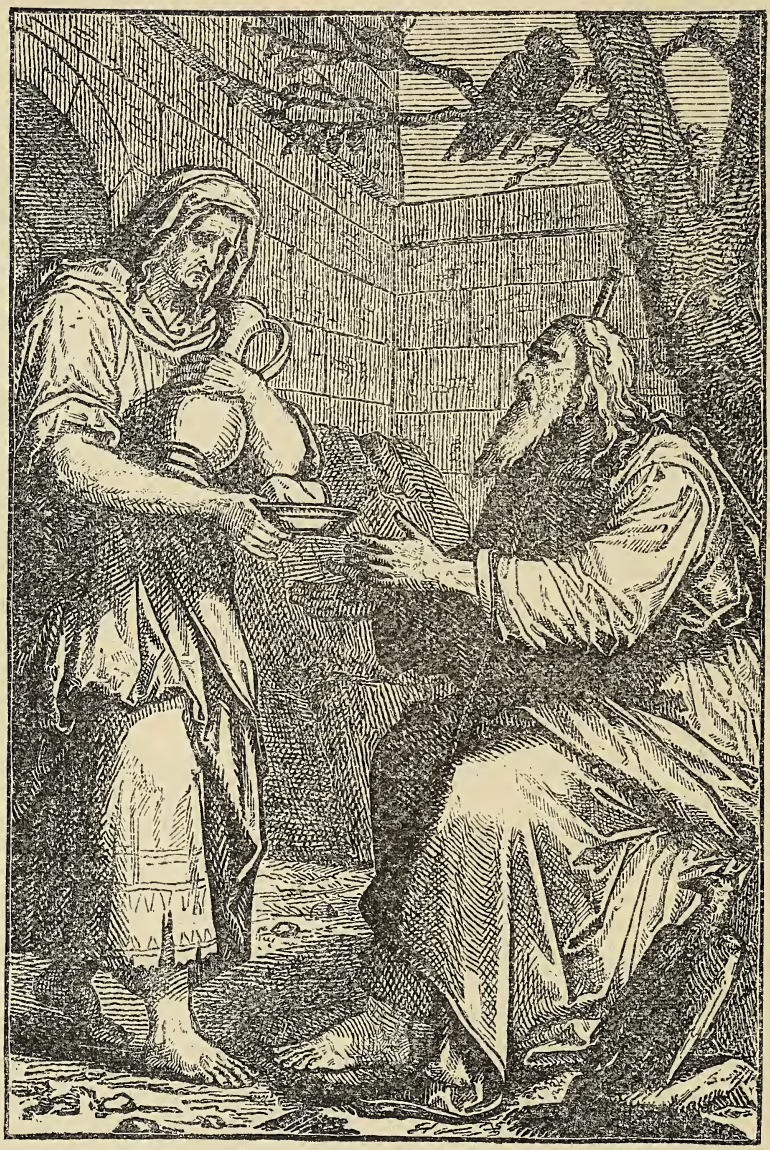

ELIJAH WIWAZICA AG்UYAPI KIDA.

iyohakam nicinkśi kici niś śpanic̣iya wo: Wakantanka heya ce; Koka kin ohna ohinni aǵuyapi niciyanke ça, wihdi ojuha kin nakun ohna ohinni 
wihdi niciyanke kta ce; qa ecen Wakantanka maka kin de ake amagiajuye kta ce, eya.

Unkan wiwazica qon he wicada he. Han, wicada. Hecen Elijah tokaheya ag̉uyapi kicağe ça, hehan iye ehake cinhintku kici śpanic̣iya. Unkan hecen anpetu otoiyohi koka kin ohna ake ag่uyapi iyecen kiciyanke c̣a, nakun wihdi iyecen kiciyanka iyekiya ece.

Unkan Elijah wiwazica onśike cin he ti kin en un; wankan tipi wan ekta oti. Hecen wiwazica kin he taku waśte iyeiçiye, Elijah hen un kin heon. Hecen Wakantanka iś ag̉uyapi qa wihdi ko ake oicahikiciciya ece. Qa nakun Elijah wiwazica kin ohinni Wakantanka oie okiyaka. Ecin wakağapi ohoda kin heceedan nahon icaġa; tuka Wakantanka oie kin nahonkiye.

Unkan wiwazica qon he woiyokiśice wan tanka akipa. Anpetu wan en cinhintku hokśidan qon he wayazanka; tuka nina hin wayazanke ça ecen ța. Wiwazica onśika nina iyokiśica. Unkan Wakantanka he teye cin ecin. Qa Wakantanka canniyeye ça heon hecakicon kecin. Qa ehanna eśta Elijah wati kin den u śni, śta keś, ecin. Qa unhanketa Elijah en i, qa icanksiya okiya. Unkan Elijah heya, Nicinkśi kin maqu wo, eciya.

Wiwazica kin hokśiyopa te cin adokiksohan yanka. Unkan Elijah hokśiyopa te cin icu, qa iye yanke cin ekta aki; qa tohe kin akan eonpa. 
Qa hehan Elijah Wakantanka cekiya; qa heya, Itancan, Wakantanka mitawa, wiwazica kici watı kin woiyokiśice kin de akipeyaya he; hokśiyopa kin he niye țeyaya he, eye ça, hehan hokśiyopa ța wanke cin en yamni akihde yugate ça hehan

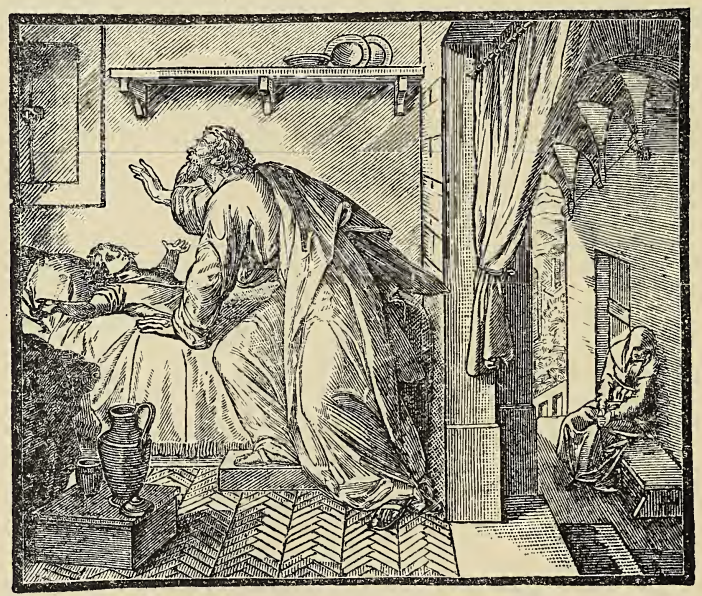

ELIJAH WIWAZICA CINCA KIN NIKIYA.

ake Wakantanka cekiye: qa heya, Itancan, Wakantanka mitawa ceciciya ce, hokśiyopa kin de ake naği kin en hdiyohpeya wo, eya.

Unkan Wakantanka Elijah tawocekiye kin nahon. Hecen hokśiyopa qon ake naği kin hdiyohpaye ça hecen kini.

Hecen Elijah toke hin iyuśkin cen eca. Wakantanka onśida qa tawocekiye kin ayupte ça, hokśiyopa wan ake kini kin heon. Wakantanka 
iśnana iyotan, qa taku owasin econ okihi kin he wiwazica kin sdonyeya cin kin heon Wakantanka hecon.

Hehan Elijah hokśiyopa qon hunku en kicicai, qa heya; Nicinca kin wanhdaka wo, kini ce, eya. Unkan wiwazica qon heya; Wakantanka tawaniyan e sdonwaya ce; qa Wakantanka eciyatanhan taku omayakidake cin owasin wowicake ce, eya.

Oyate kin tona Wakantanka cekiyapi eca he nahon. Tuka hokśiyopa wan niye cin he ohinniyan hecon śni. Tuka tona Wakantanka waśtedakapi kin hena iye ti kin heciya e niwicaya ece.

Hokśipidan, wicinyanpidan, tokin Wakantanka waśteyadakapin ; hecen tohan nițapi kinhan nitancanpi kin hena maka kin den nihnakapi kte, tuka ninaggipi kin hena hehan Wakantanka hduhe kta; qa tohan anpetu ihankeya kinhan, hehan Jesus Messiya mahipiya śapa wan ohna kun u, qa mazayahotonpi tanka wan yahotonpi nayahonpi kinhan, hehan ninagiipi kin hena nitancanpi kin en kiyohpaye kta, qa hehan wicatancan teca duhapi kta. Anpetu kin he en toke hin wowiyuśkin tanka kta cen eca. 


\section{WOONSPE 26.}

ELIJAH, QA WAHNA WOŚNAPI NONPA KIN.

Elijah wiwazica ti kin hen tehan un. Ahab wicaśtayatapi kin tawicu Jezebel kici wanyakapi kinhan ktepi kte cin heon naiçihma un: Makoce kin tankaya Ahab odewicakiya, tuka tuwedan iyeye śni. Unkan he tokeca e Ahab Elijah canniyeya he. Wakagapi cekiye cin heon Wakantanka canniyeya e Elijah okiciyake cin heon. Jesus iś eya maka akan un qehan, wicaśta wahtanipi kin owicakiciyake. Unkan on etanhan wicaśta śice cin owasin Jesus canniyeyapi e nayahonpi qon.

Wanna woyute tonana hin aye cin heon Israel nina icanwicaśica; qa peji ko uye śni kin on Ahab wicaśtayatapi kin taśunke tawanunyanpi ko ota kiṭa.

Hehan Elijah Israel onśikapi kin on icanśice ça, ake Wakantanka maka kin amaġajuye kta e icekiya. Unkan tawocekiye kin Wakantanka nahon, qa ake kohanna magajuye kta, keciya. Tuka he itokam Wakantanka heciya; Ahab wicaśtayatapi kin en ihdoi qa ikpazo wo.

Unkan Elijah wicaśtayatapi kin en ihdoye kte cin kokipe kta he. Tuka Wakantanka he econ si e econ; qa iye Wakantanka tanyan hduhe kte cin 
ELIJAH, QA WAHNA WOŚNAPI NONPA KIN. 115

Elijah sdonkiya. Qa hecen wiwazica wan cinhintku kici om un qon ehpeya iwicayaya. Hecen wiwazica kin cante sica nace. Ecin Eiijah hen un kin heon woyute ton, qa Wakantanka oie nahon un tuka. Tuka Wakantanka hecen iwahoye, ohinui woyute ton, qa ecen ake maka kin amagajuye kta keya.

Unkan Elijah ye cin tahepi ookiye wan waśte e akipa. Ahab wicaśtayatapi kin he taokiye. Ookiye kin he wato ode. Unkan ookiye kin he Elijah wanyake cin iyekiya; qa on nina yuśinyaya. Wanna wanakajatanhan Kanan makoce kin en tuwedan Elijah wanyake śni. Unkan ookiye waśte kin he Elijah itokam itemakipusdiya ehpeiçiye ça, Elijah Itancan mitawa he niye he, eya.

Hehan Elijah, De miye ce; Ahab wicaśtayatapi nitawa kin okiyag ya wo, den waun ce, eya. Tuka ookiye kin hecin, okinni iyaye cin hakam Wakantanka Elijah tokan iyayeye cinhan, hecen wicaśtayatapi kin Elijah wanyake śni kinhan, on sihda, qa iye kte kta kecin, qa tawațenye śni.

Tuka Elijah tohanyan wicaśtayatapi kin en hi śni kin hehanyan en un kta keciya; hehan ookiye qon wicada qa iyaya.

Ahab is tokan iye taśunke wato owicakide un ; hehan ookiye qon ekta i, qa, Elijah wanniyake kta anipe ce, eya. Hecen Ahab ekta hi.

Ahab Elijah wanyake çehan kig̀e, qa, Wicaśta 
wan Israel oyate nagiyewicaye ciqon he niye he, eciya. Wakantanka magajuye sni kin, Elijah he hecen icekiye ça on hececa, Ahab kecin.

Hehan Elijah iś heya; He miye śni ce, tuka he niye niohan sice cin on, wokakije kin de yakaga ce. Wakantanka anayaġoptan śni, qa wakagapi e ohoyada kin heon.

Hehan Elijah Ahab token econ kta cin qon he eciya, qa heya; Baal wakagapi kin en waayatapi kin hena owasin paha wan tehanwankantu ekta yewicaśi wo.

Unkan Ahab Elijah token econ śi qon econ he. Han, ecen econ. Magiaju kta cin kin heon; okinni Elijah magaju kta e Wakantanka icekiye cinhan ecetu kta ecin. Okinni Ahab Elijah kte eśta heon iyotan Wakantanka tohinni maġajuye kte śni, ecin kin heon kokipa.

Hehan hanhanna hinca Elijah Baal wakagapi kin en waayatewicakiyapi kin hena om paha kin ekta i, qa oyate kin wicota hinca nakun ekta ai; qa Ahab wicaśtayatapi kin en opa, tuka tawicu Jezebel e ope śni.

Unkan Elijah oyate kin token ewicakiye kta he. Wakantanka eceedan wakan qa iyotan kin he oyate kin sdonyapi cin, qa hewicakiya; Wakagapi waayatapi kin hena tatanka wanji ktepi, qa wahna wośnapi kin akan ehnakapi; qa hehan iye wakağapi tawapi kin, Baal hee, wankantanhan 
ELIJAH, QA WAHNA WOŚNAPI NONPA KIN. 117 peta uye c̣a, huhnahye sipi kta ce. Qa hehan miś tatanka tokeca wanji ktepi, qa wahna wośnapi kin akan ewahnake kta ce. Qa Mitawakantanka he wawakiyuśna e mahpiya kin eciyatanhan peta uye ça, huhnahye kta ce. Kinhan hehan iś taku wakan yuhapi kin, qa miś Mitawakantanka, unma tukte Wakantanka iyotan kinhan sdonyapi kta ce, ewicakiya.

Unkan oyate kin Elijah taku ewicakiye cin he iyokipipi qa ecen econpi.

Hehan Elijah Baal waayatapi kin iye tokaheya econ wicaśi. Hecen tatanka wan ktepi qa wahna wośnapi kin akan ehnakapi, qa can ko en ejupi. Qa hehan wakagapi yuhapi qon he cekiyapi, qa huhnahye sipi, qa panyanhan heyapi; $\mathrm{O}$ Baal naunhion miye, eya kipanhanpi. Qa wakagapi kin he cekiyapi eca, hena hecekcen śkanpi ecee kin iyecen wahna wośnapi kin ihdukśan ipsicapi, qa, O Baal, naunhon miye, eyahanpi, ecen wihiyayedan akenonpa apa. Wanna nina mdokitapi, tuka nakun hehanyan kipanhanpi, O Baal, naunhion miye.

Hehan Elijah hewicakiya; Ecaaś, nitawakantankapi kin nina wohdaka un nanihonpi śni nace; qa iś wotihni iyaya nace; qa iś icimani iyaya nace ; qa iś iśtinma nace ; nina kipanpi qa hduhica po, ewicakiya.

Unkan Elijah he awicaka e heya he. Hiya, 
Baal kin he taku śni e sdonya; tuka oyate wacintonpi śni kin hena he wakagapi kin hecen kagapi, qa he Baal eya caśtonpi.

Nakun iyowinye sni waayate wicakiyapi kin hena Baal peta uye kta icekiya hanpi, qa ecen unhanketa isan ko on baiçispapi, qa weiçiyapi ; hecen Baal iyokipiyapi kta kecinpi. Qa ecaeś iye tawakantankapi kin wacinhinyanza on etanhan wepi kin e waśtedake kta kecinpi. Heconhanpi ecen wiyotanhan sanpa, qa wanna yamni apa, tuka hecen takudan okihi sni.

Hehan Elijah iś ito, Wakantanka mahpiya kin eciyatanhan peta uye kta e icekiye kta keya. Qa hehan wahna wośnapi wan kağa; inyan akenom witaya ehnake ça, hehan can qeya akan eju, qa hehan tatanka qon he akan ehanka; qa hehan oyate kin mini koka akenom wahna wośna kte cin en akaśtan wicaśi. Paha kin he ihukuya wakpadan wanka hetanhan mini kin tokśupi. Qa wahna wośnapi kin ihdukśan maka qapi. Hecen maka oqe kin he mini ojudan linca; qa wahna wośnapi kin nakun nina spaye hinca.

Unkan he tokeca e Elijah wośna kte cin he mini akaśtan wicaśi he.

Wahna wośnapi kin en peta tuktedan yanke sni kin he oyate kin sdonyapi kta cin kin heon hecon.

Hehan Elijah wahna wośna kte cin itokam 
ELIJAH, QA WAHNA WOŚNAPI NONPA KIN. 119

inajin, qa Wakantanka cekiya, qa oyate kin ihdukśan najinpi.

Kaken eya; Itancan, Wakantanka, Abraham, Isaak, Jakob, Tawakantankapi ; Wakantanka qon anpetu kin de oyate sdonniyanpi kte, qa nitaokiye namakilion ye. O Itancan namahion ye.

Unkan Wakantanka Elijah tawocekiye kin nahon he. Han, nahon, qa mahipiya kin eciyatanhan peta uye ça wakiyuśna kin huhnahya, inyan qa mini ko owasin huhnahya.

Oyate kin he wanyakapi kin on wicayuśinyaya: qa owasin ite makipusdiya elipeiçiyapi, qa, Itancan he Wakantanka ee, Itancan he Wakantanka ee ce, eyapi.

Hehan oyate kin Baal he taku śni e wanna sdonyapi. Hecen Elijah Baal en waayatapi kin hena wicayuzapi, qa wakpadan kin ekta awicayapi, qa wicakte wicaśi. Hecen waayatapi kin hena wakpa ekta awicaipi, qa mazasagye on wicaktepi, qa wepi kin owasin wakpa mini kin icicahi iyayapi.

Waayatapi kin hena oyate kin Baal ohodapi kin hena he waonspekiyapi kin heepi, heon etanhan owasin țapi kta e Wakantanka wicakonza.

Oyate kin maǵaju kta nina cantokpanipi. Wanna Wakantanka mahpiya tanhan peta uye cin on nakun magajuya okihi e oyate kin tanyan yukcanpi. 
Iho, hokśipidan wicinyanpidan, Wakantanka maka kin de amaggajuye cin on wojupi icahyeunkiyapi. Wakantanka wan tanka kin tuwe iyececa he. Mahpiya tanhan peta uye ça huhnahunyanpi okihi ; tuka towaonśida tanka kin on onśiundapi.

\section{WOONSPE $2 \pi$.}

\section{ELIJAH, QA MAǴGJU KIN.}

Hokśipidan, wicinyanpidan, Baal en waayatapi kin hena token wicaktepi kin he wanna nayahonpi. Hehan wanna Wakantanka kohanna magajuye kte cin Elijah he sdonya; hecen Ahab ecen okiyaka; qa hehan wote ça wayatke si. Heon paha kin he icahda Ahab wote ça wayatkan. Tuka Elijah e wote śni qa wayatke śni paha kin akan Wakantanka cekiye i; patuś kuceyedan inajin qa cekiya.

Elijah taokiye wan kicica. Unkan Elijah wocekiye eye cin icunhan taokiye kin miniwanca kin iwankam malipiya kin ekta tehan etonwan najin si. Elijah Wakantanka mahipiya śapa wanji uye kta cin; kinhan hetanhan on maǵaju kta. Unkan ookiye qon takudan wanyake śni, keya. Hehan Elijah taokiye kin śakowin akihde ekta etonwe si. Unkan econ kin hehan mahpiya śapa wan cistinna wicanape kin hinskokeca wanmdaka ce, eya. Elijah tawocekiye kin Wakantanka nakicilion 
e sdonkiya; qa mahipiya śapa kin he tanka aye ça hetanhan on magiaju kte. Hehan Elijah taokiye kin Ahab okiyake śi. Wanna kohanna maġaju kta e iye tacanpahmihma kin okipe ca, iye ti kin ekta dus hde śi. Hecen Ahab canpahmihma okipe ca kihda, token okihi kin hecen dus hda.

Unkan Wakantanka Elijah waśagye ça nakun duzaheya. Hecen suktanka kin isanpa duzahan. Ahab canpahmihma ope ça Elijah e inyanka; icunhan nina magiaju; tuka Elijah e tokaheya otonwe kin iyohi.

Wanna magiaju kin on oyate kin wiciyuśkin tanka. Cokan puza hiyeye cin owasin mini ojudan icu; wato puze cin owasin śtunka icu; qa maka qoze cin owasin panpanna icu; qa wanna wojupi owasin icag̉e kte cin oyate kin sdonyapi. Iho, Wakantanka wacantkiye cin he hee maggajuye. Iho, hecen oyate śicapi kin ayuśtanpi qa Wakantanka e waśtedapi iyececa.

Ahab iye totonwe kin ekta ki kin, hehan Baal takudan okihi śni, qa Wakantanka e malipiya tanhan peta uye cin hena owasin iye tawicu Jezebel okiyaka; qa Elijah token waayatapi kin țewicaye cin he nakun okiyaka.

Hetanhan Jezebel Wakantanka e ohoda kta iyececa; tuka ake nakun econ śni, qa Elijah nina canniyeya, qa ihanhanna kinhan kte kta keye ça okiciyag yewicaśi. 


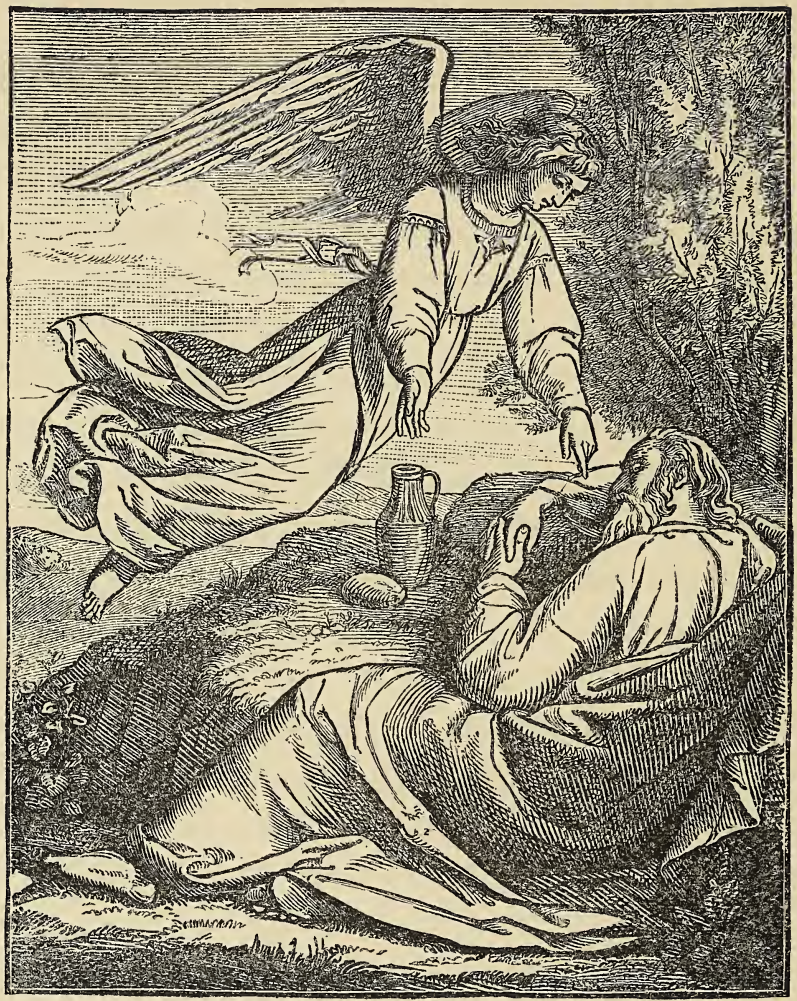

MAḦPIYA OHNIHDE ELIJAH WODKIYA.

Hehan Elijah he nakilion kin on kokipa, qa icunom napa. Kanan makoce kin opta ye ca, ecen hewoskan makoce kin ekta i. Elijah taokiye qon kici ye śni, iśnana iyaya.

Elijah hewoskan makoce kin en iśnana un kin, ecen unhanketa can wan ihukuya iyotanke ça hen 
Wakantanka cekiya, iye țe kta icekiya. Unkan he tokeca e Elijah hecen cante sica he. Jezebel kte cin kin heon, qa Jezebel olian śice cin ayuśtan śni, qa ake nakun sanpa oyate kin wakagapi ohodawicakiye kta kecin kin heon iyotan icanśica. Tokin oyate kin owasin Wakantanka e waśtedakapi keś ecin.

Elijah can wan ihukuya wocekiye eye ciqon ayaśtan qehan, ecehnana en iśtinma. Unkan iecadan tuwe oyutan. Unkan he tuwe he. He mahipiya ohnihde. Unkan, najin qa wota wo, eciya qehan, en etonwan, unkan inyun, wanke cin icahda peta, qa en ağuyapi śpanyan yanka, qa pa kin icahda janjan mini ohna han wanyaka. Mahpiya ohnihde kin hena wiyeya ahikihde. Iho, tuwe Wakantanka waśtedaka eca taohnihde kin hena en ape unwicakiya ece; qa mahpiya ohnihde kin iś he econpi iyokipipi.

Elijah wote ẹa wayatke ẹa ake iśtinma. Tuka ake iecadan mahpiya ohnihde kin yuhica, qa, Kikta qa wota wo, wanna kohanna téhan de kta ce, eciya. Hecen Elijah wote ça wayatkan, qa hehan hewoskan makoce kin ehna tehan ya. Unkan mahpiya ohnihde woqu qon he iwakiśaka, qa hecen anpetu wikcemna topa hehanyan wote śni qa wayatke sni. Unkan he wowapetokeca tanka he. Wakantanka cin unkans, oyate kin woyute codan eśta niwicaya okihi. 
Jesus nakun maka akan un qehan, anpetu wikcemna topa wote śni qa wayatke śni.

Iho, hokśipidan wicinyanpidan, Elijah iyokiśica, unkan icunhan en Wakantanka onśida qa token kicanpte cin de nayahonpi. Qa wanna de ota nahonciyapi, tohan iyoniciśicapi kinhan, hehan nina hoyekiya po, kinhan wokicanpte niçupi kta ce.

\section{WOONSPE 28.}

ELIJAH, QA ELIŚA KICOPI QON.

Elijah anpetu wikcemna tom hehanyan wote sni hewoskan makoce kin en iśnana un, ecen unhanketa imnija olidoka wan en i. Unkan ohdoka kin he mahen i, qa en iśtinma. Olidoka kin he mahen un kin icunhan, Wakantanka okíya qa he tokeca on hen i kin he iwangia. Elijah token on hecen un kin he wanna nayahonpi. Wicaśtayatapi winohinca ohan śce cin heon Elijah Kanan makoce kin etanhan tokan iyaye cin Wakantanka he sdonya, tuka he iwanga cin, on iwang.a.

Hehan Elijah iś ayupta, qa Israel oyate kin Wakantanka Koka tawa kin he yutakunipi śni, qa Wakantanka waayata tawa kin wicaktepi, qa miśnana omakaptapi; unkan ake nakun miś maktepi cinpi ce, eya. 
Hehan Wakantanka Elijah tankan ku śi, qa he kin akan inajin si. Hecen Elijah tankan hdicu, qa lie kin akan inajin. Hehan Wakantanka he kin he en tate wan tanka iyayupteya. Hecen he kin huhuzahe ça imnija kin namdeca. Qa peta wan nakun en iyayupteya. Wakantanka towaśake kin Elijah kipazo kin heon hecon. Hecen taku tehika eśta etanhan niye kte cin he Elijah sdonkiye kta; qa tona Elijah kte wacinpi, qa oyate śicapi kin hena Wakantanka kakiświcaya okihi.

Hehan Wakantanka Elijah ahbayedàn okíya. Unkan Elijah wicaho kin he nahon kin hehan taonhohda kin on ite aihdahpe ça ohdoka kin mahen inajin.

Wakantanka Elijah waśtedaka, heon kicanpta. Tuka Elijah iś Wakantanka ecedan taku Wakantanka kin hee, he kiksuye cin, heon ite nakilima. Mahpiya kin ekta Wakantanka oyankeye cin en ihdukśan mahpiya ohnihde najinpi kin hena iye hupahupi kin on ite aihdahpa najinpi.

Iho hokśipidan wicinyanpidan, niś tohan Wakantanka ceyakiyapi kinhan, ihnuhan ican tokan ewacannipi kin. Taku Wakan wanjidan tanka kin hee ce, he kiksuya po; Wakantanka ohinni wanniyakapi ce.

Unkan Wakantanka Flijah tokeca e hen un kin he ake iwangia. Hecen Elijah iś ake oyate 
sicapi kin hena kte wacinpi kin heon hen un kin he ake okiyaka.

Hehan Wakantanka oyate sicapi qa wakagiapi ohodapi kin hena kakiświcaye kte cin he Elijah okiyake. Qa Wakantanka heya, Oyate kin iyuhpa wakaggapi kin ohodapi śni ce; Israel oyate en wicota tohinni Baal itokam canpeśka makehde inajinpi śni ce ; qa iiputakapi śni ce, eya.

Elijah hecin, oyate kin tuwedan Wakantanka waśtedake śni, qa iśnana hin hececa maka akan un kecin. Tuka wicota Wakantanka waśtedakapi kin he nation kin on nina iyuśkin. Wakantanka tona waśtedakapi kin hena sdonwicakiya, qa hena wicayawa, qa ohinni wicakiksuya.

Hehan Wakantanka Elijah heciya; Wicaśta wan Eliśa eciyapi kin he ekta ya wo, qa ihepi on ecakicon wo, kinhan he waayata heca kta ce, eya.

Hecen Elijah imnija olidoka qon etanhan iyaya, qa Eliśa ode aya. Unkan inyun, wicaśta wan tatanka akicaśka akenom on mahyumdu en i, unkan Eliśa hee.

Unkan Elijah taonliolida tanka hduśdoke ça wicaśta qon tapetepa-tanhan akalipa. Unkan Elijah token on hecon kin wicaśta kin sdonkiya; qa hecen tatanka tawa qon owasin ehpeya kici ye kta keya. Tuka ito ate qa ina iiwicaputag mde kta ce, iyowinmakiya wo, eya.

Unkan Elijah iyowinkiya. Hecen wicaśta qon 


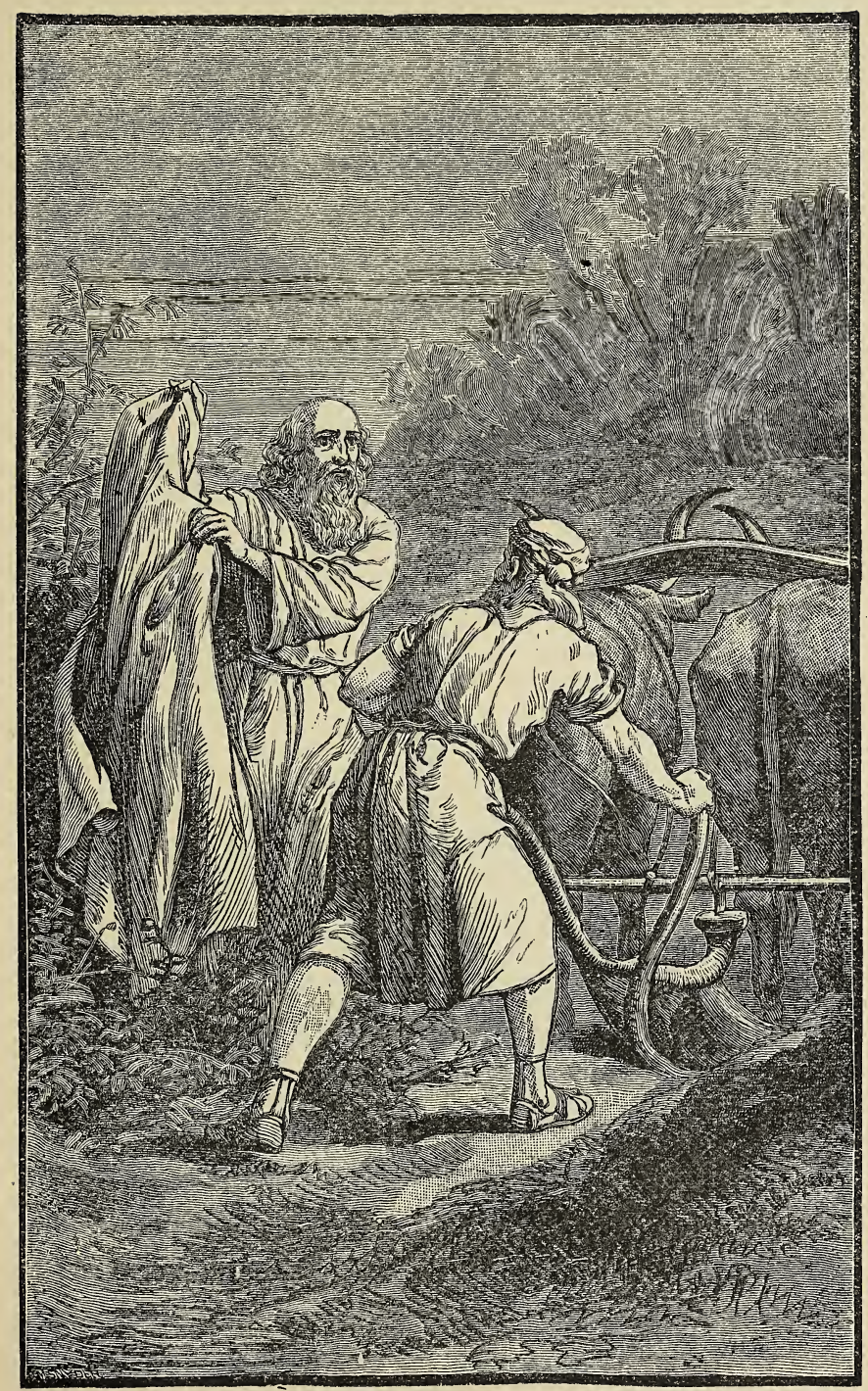

ELIJAH TAONHOH'DA ELISA QU. 
tiyata ki, unkan tatanka qon etanhan nom kikte, qa can wanapin kin hena etanhanna on ceti, qa tado kin ceom śpanye ça, kodawicaye cin owasin om mniciya. Qa hehan ti ehpeya Elijah kici iyaya, qa hecen Elijah taokiyeya.

Elijah kici unpi waśte nace. Han, kici unpi waśte. Ecin ohinni Wakantanka cekiya un, qa oie kin nakun ohinni nalionwicaya. Tuka wicaśta sicapi kin hena Elijah canniyeyapi. Hecen wicaśta kin he iś eya Wakantanka waśtedake śni unkans, Elijah kici un iyokipi kte śni tuka. Tuka Wakantanka waśtedake cin heon iś eya Wakantanka waśtedake ça yawaśte.

\section{WOONSPE 29.}

\section{ELIJAH, QA NABOT TAWOJU KIN.}

Ahab tohian śica wanji ake nayalionpi kte. Oyate wakagiapi ohodapi kin hena wicolian śica ota nakun iyahna yuhapi.

Ahab wijica linca; tipi waśte nom yuhe cin, unma itehanyan otonwe wan ekta han. Unkan tipi kin en icahda magga wan en hastanyanka ota ojupi. Unkan wicaśta wan Nabot eciyapi hena he tawa. Unkan Ahab hastanyanka ojupi kin hena cin linea, qa iye tamağa kin en ehde cin, qa 


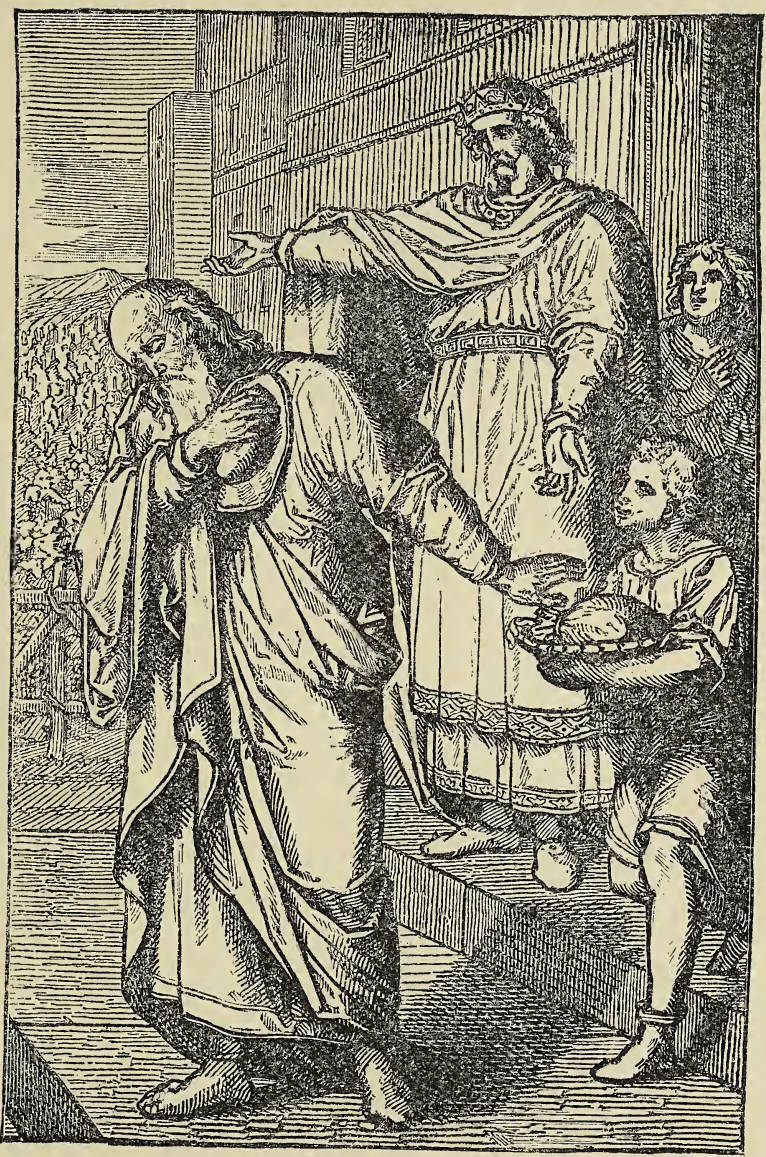

NABOT TAWOJU WIYOPEYE KTA WICADA ŚNI.

hecen Nabot en i, qa, Hastanyanka ojupi kin he mayaqu kinhan, tokeca wan waśte ciçu kte, qa iś mazaska e yacin kinhan, cic̣u kta ce, eciya. 
Tuka Nabot iś ayupte ça, Ate hena maqu e wiyopewaye kte śni ce, eya.

Hecen qu śni. Unkan heon Ahab nina śihda, qa tiyata ki, tuka icanśice cin on wote śni makan wanke ça, paptanyan iyeiçiya wanka. Ahab wacinton śni. Wanna taku waśte ota yuha, tuka nakun sanpa cin. Tona Wakantanka waśtedakapi śni kin hececapi. Taku ota tonpi eśta tohinni imnanpi śni ece.

Ahab cante sice cin hehan Jezebel wanyake ca, Tokeca e wayate śni he; taku icanniśica he, eciya. Unkan Ahab, Nabot hastanyanka wojupi yuhe cin he wakida, tuka wiyopeye kte śni, keya ce, eya. Qa Jezebel ayupta : Miye he cic̣u kte ye.

Jezebel hastanyanka wojupi kin he token icu kta he. Tohan sice kte cin he awacin yanka. Nabot kte kta e he ka. Qa hecen wayaco qeya ohan śicapi e Nabot ti kin icahda tipi, unkan he wowapi wicakicağa, qa hewicakiya; Wicaśta wicaśtapi śni etanhan heye wicayaśipi kta ce ; Nabot Wakantanka ośtehda qa wicaśtayatapi kin; qa hecen heon dacopi qa țeyayapi kta ce, ewicakiya.

Unkan hecen Jezebel wowapi wicakicag่e cin wanyakapi qehan, token econ wicaśi kin ecen econpi. Qa Nabot Wakantanka ośtehda, qa nakun wicaśtayatapi kin ośtehda ce, eya wicaśta nom oitonśni woyag wicaśipi. Qa hecen te kta yacopi. Qa hecen otonwe tankan aipi, qa hehan 
inyan on kinin ktepi. Nabot we kin wanna maka kin apsonwahe, qa śunka we kin sdipapi. Wakantanka Nabot we kin wanyaka, qa he śihda

Wanna Nabot ktepi kin Jezebel okiyakapi. Hehan Ahab heciya, Nabot wanna ta ce, ekta ye ca hastanyanka wojupi kin icu, eya.

Hecen Ahab ekta i, qa hastanyanka wojupi qon icu, qa iye tamagia kin en ehde.

Hehan Wakantanka Elijah okiyaka, Ahab token econ kin he. Qa Wakantanka Elijah heciya; Anpetu wan en Ahab we kin śunka sdipapi kta ce; en ye ça okiciyaka wo, eya.

Hecen Elijah Ahab en i. Ahab Elijah wanyaka cin nina icanśica; wahtani kin okiciyake cin heon wanyag kapin. Qa Ahab heya, Tokamaye ciqon ake iyemayaya he, eciya.

Hehan Elijah iś heciya; Wakantanka heya ce, Anpetu wan en, niwe kin śunka sdipapi kta ce; qa anpetu wan en Jezebel tancan kin iś śunka temyapi kta ce; qa nicincapi kin iś tancanpi kin śunka zitkadan ko wicayutapi kła ce.

Ahab wicoie kin de nalion kin on tawokoyake kin aihdupote c̣a waśihda, qa akihanic̣iye ça iyahnana mani.

Hehan Wakantanka Elijah heciya; Ahab mitokam token onśihan kin wandaka he. Mitokam onśilian kin heon etanhan ito, wokakije kin he kohanna en awau kte śni ce, tohanyan ni un kin 
hehanyan; qa țe cinhan hehan cinca kin tancanpi kin sunka zitkadan ko wicayutapi kta ce.

Wakantanka waonśida hinca, heon Ahab cinca om kakiświcaye kte ciqon ito, tehan econ kte śni. Wakantanka towaonśida ecedan tanka. Han, tuka tona anag̉optanpi śni, qa śicapi eca unhanketa Wakanśica tapeta kin en oiyohpewicaya ece.

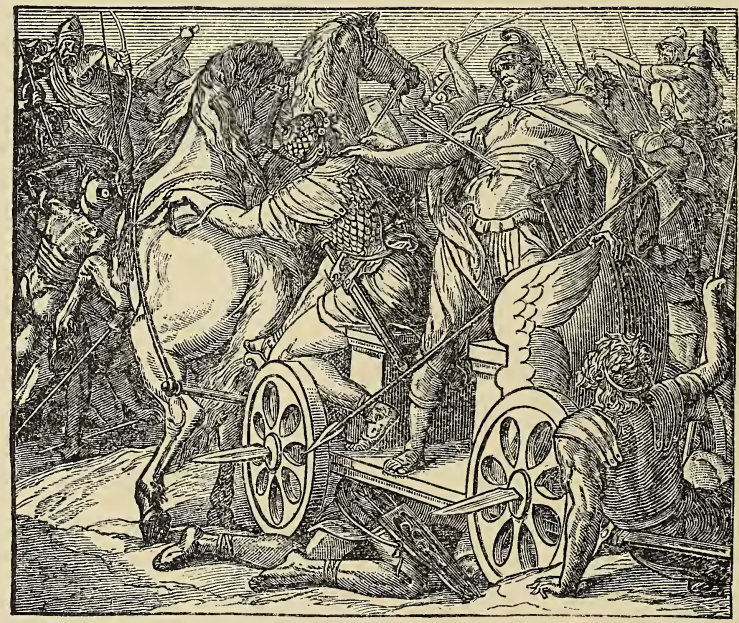

AHAB TE CIN.

Ahab Saul iyececa. Cante sica tuka he walitani iyopeiçiya on hecece śni. Wakantanka kakiśye kta ikope cin hecedan on hececa.

He iyohakan itehan hehan Ahab okicizeta i. Oyate wan Kanan ikiyedan tonwanyanpi he wicakis i. Ahab canpahmihma waśte hinca ohna i. 
ELIJAH, QA AKICI'A 'IANCAN YAMNI. 133

Ilehan wanhinkpe on opi, qa oo kin nina we au. Hecen śuktanka kaliape cin Ahab iye ti kin ekta ahda. Tuka ahde cin tahepi Ahab ța. Tuka ecehna iye ti kin en akipi, tacanpahmihma kin we

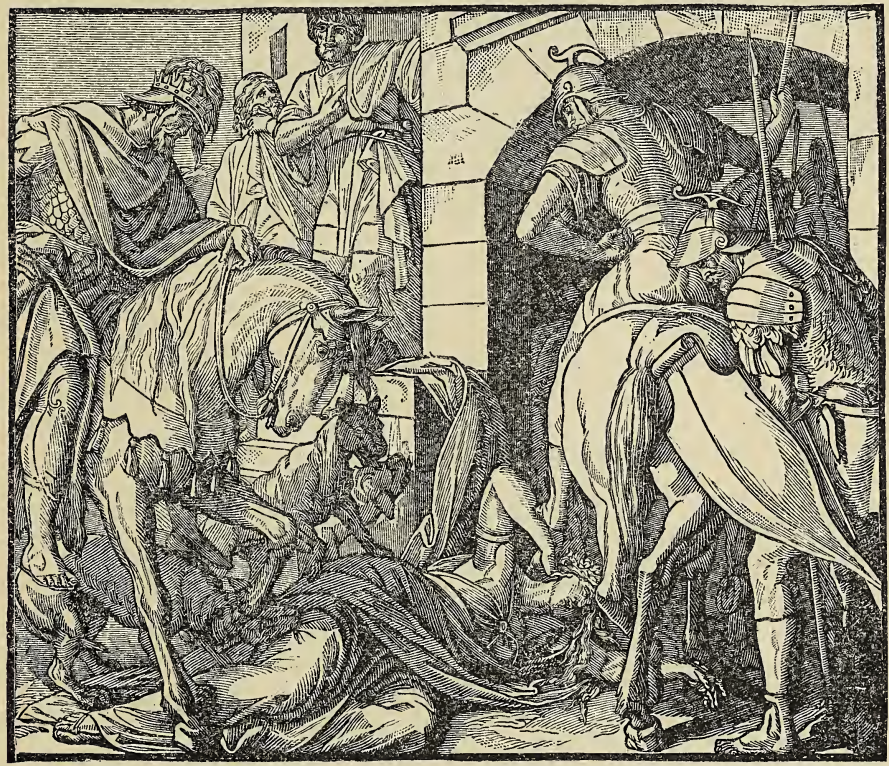

JEZEBEL TE CIN.

ojudan. Hecen ookiye kin canpahmihma kin miniyata yujaja i icunhan śunka kin Ahab we kin sdipapi.

Wakantanka heye ciqon owasin iyecetu. Hehan Jezebel tohan śice cin on iś taku e akipa he. He iyohakam iś hehan ktepi. Akicita tancan wan, 
Jezebel taokiye kin, Jezebel owanyeye eciyatanhan kahoya hiyuye wicaśi ; unkan econpi. Hecen Jezebel we kin kaśtan hinlipaya. Unkan akicita tancan taśunke kin Jezebel tancan kin amani kiya. Hehan akicita tancan kin wote i. He icunhan Jezebel tancan kin śunka yutapi, qa temyapi, qa huhu ece oyaptapi. Winohinca śice ça Ahab tohan śice cin nina iyopaśtake ça, Wakantanka waayata tawa waśte ota tewicaye ciqon iś ito hehan ihanke.

\section{WOONSPE 30 .}

\section{ELIJAH, QA AKICITA TANCAN YAMNI.}

Ahab te cin hehan, ake cinhintku wan wicaśtayatapi, Ahaziah eciyapi. Iśs eya he hunkakewicaya olian sicapi kin iyewicaceca, qa is eya wakagapi cekiya.

Ahaziah wicaśtayatapi kin iecadan nina kiunni. Wankan tipi wan ekta un, unkan heciyatanhan owanyeye etanhan ohinlipaye ca ksuweya. Qa hecen heon țe kta kecin. Qa țe kte ça iśs asni kta hecinhan he sdonkiye kte hinca. Qa hecen iye ti kin itehan wakag̣api wan hdepi, he eya wanna nahon, unkan he ekta taokiye yewicaśi.

Hokśipidan wicinyanpidan, niś tohan waniyazankapi eca, anisnipi, qa iś anisnipi kte śni, unma 
tukte kin tuwe sdonya he. Wakantanka iśnana he sdonye. Hecetu, tuka Ahaziah witkotkoke cin wakaġapi wan e he wokiciyakapi kta kecin.

Unkan ookiye kin yapi tahepi wicaśta wan akipapi. Tuka he tuwe kin sdonyapi śni. Wicaśta kin he wakihdaka wokoyake hin tonyake koyaka; hin kin e akapatanhan un, qa ipiyaka wan aipiyaka. Elijah hee. Wakantanka Elijah ookiye kin he owicakiye śi.

Hecen Elijah ookiye kin hewicakiya, Ahaziah ni, qa iś te kta hecinhan he sdonya cin, qa on wakagapi wan wahoye cin he Wakantanka sihda ce. Hecen tohinni wanke cin hetanhan najin śni ecen te kta, Wakantanka keya ce, eya.

Unkan ookiye kin yuśinyayapi; wicaśta wan akipapi, unkan taku on toki yapi kin owasin sdonye cin heon. Hecen hetanhanna icicawin kipi, qa waayata taku oyake cin he Ahaziah hośi kakipi.

Unkan Ahaziah Elijah hee nace ecin. Ahaziah Elijah waayata heca kin he nahon, qa wicaśta kin he taku wicaśta ka he, taokiye kin eya iwicawanga.

Tawokoyake kin hinton, qa ipiyaka wan aipiyaka ce, taokiye kin eyapi. Hehan Ahaziah, Elijah hee ce, eya.

Ahaziah Elijah nina canniyeya, te kta keciye cin heon. Tuka Elijah wowapetokeca econ okihi 
kin he sdonya; heon akeś wanyaka cin. Qa akicita tancan wan akicita wikcemna zaptan om Elijah huwe yewicaśi.

Hecen akicita tancan kin taakicita om Elijah paha wan akan yanka en ipi. Unkan akicita tancan kin Elijah śikśin owakiye, qa, Wakantanka tawicaśta kin, kohanna kun ku wo, wicaśtayatapi heya ce, eciya.

Unkan Elijah ayupte ça, Wakantanka tawicaśta waun hecinhan, mahpiya kin eciyatanhan peta uye ça, nitaakicita om nihulinag̀e kta, eya.

Unkan mahipiya kin eciyatanhan peta hiyu, qa akicita tancan kin taakicita om huhinag̉a.

Unkan ake nakun akicita tancan wan tokeca akicita wikcemna zaptan om ye śi. Hecen ake Elijah paha akan yanke cin en i, qa, O Wakantanka tawicaśta, kohanna kun yaku kta, wicaśtayatapi keya ce, eya.

Unkan Elijah ayupte ça, Wakantanka tawicaśta waun hecinhan, mahpiya kin eciyatanhan peta uye ca, nitaakicita om nihuhnag่e kta, eya. Unkan Wakantanka mahipiya tanhan peta hiyuye ça owasin huhnahwicaya.

Hehan ake akicita tancan tokeca ake akicita wikcemna zaptan om ye si. Unkan akicita tancan kin he qe kaken econ. Elijah yanke cin en i qehan onśikihan. Elijah itokam canpeśka makehde ehpeiciye ça, O Wakantanka tawicaśta, ceciciya 
ELIJAH, QA PEIA CANPAHMIHMA KIN. 137

ce, wani kte, qa nitaokiye wikcemna zaptan kin dena nipi kte, eya.

Hehan Wakantanka taohnihde wan Elijah heciya; Kun ye ça kokipe śni kici hda wo, eya.

Hecen Elijah akicita tancan kin kici wicaśtayatapi kin ekta ki. Ahaziah wayazanka wanka hehan Elijah heciya; Anisni kta sdonyakiya yacin, qa wakaggapi wan e wahoyaye cin heon tohinni anisni kte śni, Wakantanka keya ce, eya. Unkan iecadan Ahaziah ța.

Hehan ake wicaśtayatapi wan tokeea; tuka iś eya ake Ahab qa Ahaziah iyewicaceca, qa olian śica.

\section{WOONSPE 31.}

ELIJAH, QA PETA CANPAHMIHMA KIN.

Eya wancadan Elijah wiconte cantokpani nayahonpi qon. Tuka Elijah tohinni țe kte śni ; tanzaniyake malipiya kin ekta ye kta e Wakantanka konza.

Hehan Elijah wanna ecadan Mahpiya kin ekta ye kte cin he sdonkiya. Qa he itokam makoce kin otakiya kodawicaya unpi kin hena ekta wicai. Takodaku hena waayatapi kośka waśtepi. Elijah wanna kohanna mahpiya ekta ye kte cin waayata kośka kin hena sdonyapi. 
Eliśa nakun Elijah mahpiya ekta ye kte cin wanyake kte hinca, qa ohinni ikiyedan ece un.

Hehan Elijah Eliśa heciya; Ceciciya ce, den un ye; Itancan Betel ekta ye maśi ce, eya. Tuka Eliśa ayupte ça, Aciyuśtan kte śni ce, eya. Hecen kici Betel ekta i.

Unkan ake Elijah Eliśa heciya; Ceciciya ce, den un ye; Itancan Jeriko ekta ye maśi ce, eya. Hiya, aciyuśtan kte śni ce, Eliśa eya. Hecen kici Jeriko ekta i.

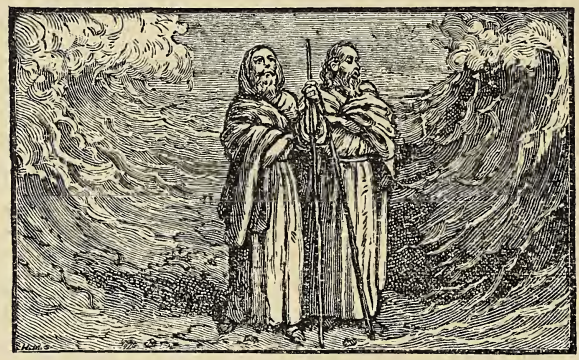

ELIJAH QA ELIŚA JORDAN EN.

Ake Elijah, Ceciciya ce, den un ye; Itancan Jordan ekta ye maśi ce, eya. Hiya, aciyuśtan kte śni ce, ake Eliśa eya. Hecen napin yapi, qa ecen Jordan wakpa kin en ipi. Unkan Elijah taonhohda tanka kin hduśdoke ẹa yupehan yus on mini kin apa. Unkan Wakantanka mini kin akipam iyayeya. Hecen Elijah Eliśa kici wakpa kin opta yapi, tuka maka pusa amani iyayapi. 
Wanna wakpa kin akasanpa unpi kin, hehan Elijah Eliśa heciya; Emayakupi śni kin itokam taku yada kinhan, ecacicon kta ce, eya. Unkan Eliśa taku e da he. Elijah waayate cin he iś eya iyecen un cin, qa Elijah tawacin kin ocowasin kida.

Unkan Elijah heya, Taku telika e yada ce; tuka emayakupi kin en icunhan wanmayadake cinhan, enicicetu kta ce; tuka wanmayadake śni kinhan, ecetu kte śni ce, eya.

Qa ake iyoopta wohdag yapi icunhan mahpiya kin eciyatanhan peta canpahmihma, qa peta sunktanka wicayukinukan, qa Elijah wamniyomni e ohna mahpiya kin ekta akiyahdapi. Eliśa he wanyake cin hehan panyanhan, O Ate Ate, eya.

Hehan Eliśa wanna takodaku ehpeya iyaye cin on nina iyokiśica. Elijah eyakupi qon hehan taonholida tanka kin en hinlipaya. Hehan Eliśa he icu qa yuha Jordan wakpa kin en hdi qehan, Elijah econ qon iyecen, iś eya onhohida kin on mini kin en apa. Unkan mini kin akipam iyaya. Hecen Eliśa maka pusa amani hdicu.

Wakpa kin akasanpa hdicu kin hehan, Elijah takodaku kin hena hen najinpi; qa Eliśa wowapetokeca econ kin he wanyakapi, qa heyapi; Elijah tawacin qon he Eliśa yuha ce, eyapi. Qa hehan Eliśa en upi, qa pa kicapsanpi. Waayata kośka kin hena Elijah oie kin woanaggoptan yanpi. 


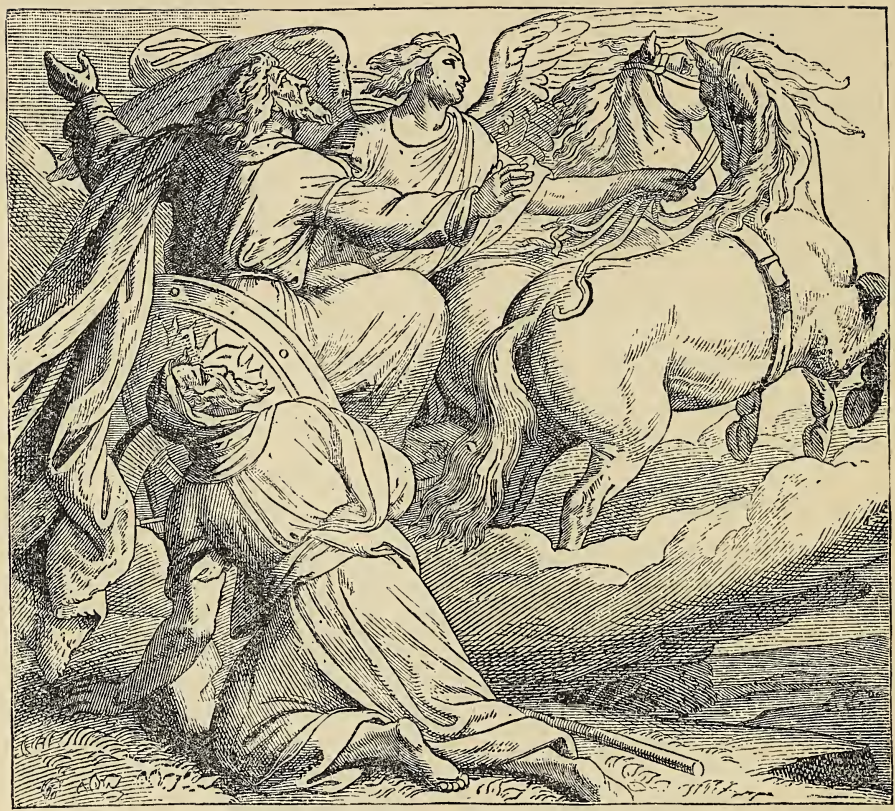

ELIJAH MAH́PIYA EKTA AKIYAHDAPI.

Hehan iś ito ake Eliśa he woanag̉optan yanpi kta. Hecen Elijah maka akan Wakantanka oie kin token yaotanin qon, Eliśa iś ito hena econ kta.

\section{WOONSPE 32.}

ELIŚA, QA WAḦANKSICA KIN.

Unkan anpetu wan en Eliśa otonwe wan Betel eciyapi, he en ya. Oyate kin he wakaggapi cekiyapi. Jeroboam wicastayatapi qon he otonwe kin 
he en ptejicadan wakağapi wan kag̉a, qa oyate kin cekiyapi. Eliśa he en ya, unkan śiceca wicota otonwe etanhan tankan upi, qa Eliśa ihahapi, qa heyapi; Pe śda wankan hda wo, pe śda wankan hda wo, eciyapi.

Elijah mahpiya ekta kihde cin heon iś eya heciyapi. Qa Eliśa oie kin nahonpi cinpi śni kin heon heyapi.

Unkan Eliśa ekta ihdamna, qa awicatonwan, qa, Wakantanka wokakije tanka wan hiyahdeniyanpi kta ce, ewicakiya. Unkan itam wahanksica nom canyatanhan hinanpapi, qa siceca wikcemna tom sanpa nonpa kin hena owasin wicayapotapi.

Hehan Eliśa Karmel paha kin ekta i, qa icicawin Samaria ekta i.

Śiceca qeya ścapi qa Eliśa ihahapi, qa on tehiya waakipapi kin de wanna nayahonpi. Hehan Eliśa winohinca onśika wan tanyan ecakicon kin he nayalionpi kta.

Winyan kin he wiwazica, qa hihnaku kin waayata heca, tuka wahpanica ecen ța. Unkan winohinca kin he Eliśa en hi qa heya; Mihihna kin Wakantanka kokipeyahan un e sdonyaye ciqon. Tuka wanna țe. Unkan ikicazo qon hee hi qa micinca nom wayaka iwicadake kta e iwicacu hi ce, eya.

Unkan Eliśa, Taku tokacicon kta he, eya. Qa hehan, Yati kin ohna taku duha hecinhan omaki- 


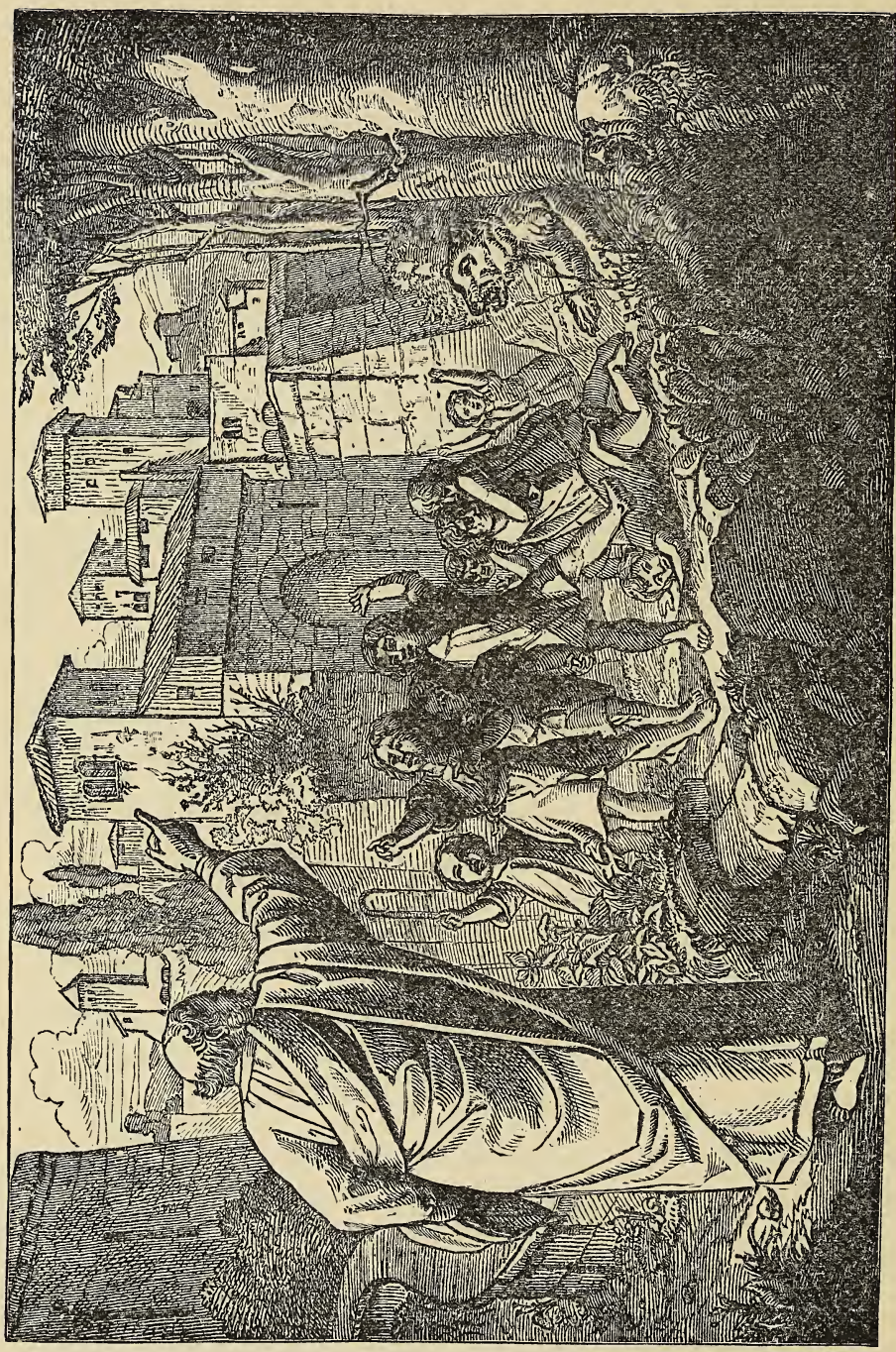


yaka wo, eciya. Unkan heya; Takudan wahnake śni makanjanjan wanjidan wihdi ohna wahde ce, eya.

Unkan Eliśa heya; Janjan ojuha qa iyokaśtan ko ota odon ye ça, hena yati kin en akihde; qa hehan tiyopa onakitake ça, nicinca om wihdi kin he ojuha kin owasin ecen okaśtan ehde wo, eciya.

Unkan wihdi tonana liince ciqon ojuha kin e ota kin henakeca ojuya okihi kta he. Winohinca kin de Wakantanka Eliśa tawicaśta kin on taku wowapetokeca econ okihi kin he sdonya, heon wicada, qa ecen econ.

Hecen ojuha iyokaśtan ko ota odon ahdi; qa hehan cinca om tiyopa onatag wihdi tonana yuhe ciqon he ojuha ota kin hena en okastan yanka. Tuka wihdi ojuha tawa qon hee ohinni wihdi okicihnaka kin hecetu wanjica. Unkan, Ake ojuha kin wanji makau, cinhintku kin eciya. Tuka iś, Wanna ojuha kin henana, owasin ojudan ce, eya. Unkan hehan wihdi kin iś nakun hekicinana.

Hehan hena token econ kte cin, he Eliśa ekta iwanh i. Unkan heciya; Wihdi kin hena wiyopeye ça, icazopi owasin hdajuju, qa tona iyaye cin hena nicinca om hduha wo, eciya.

Tho, hecen wiwazica kin cinca om toke hin wiyuśkin cen eca; Wakantanka towaonśida tanka sdonyapi kin heon. 


\title{
WOONSPE 33.
}

\author{
ELIŚA, QA WICINYANNA KIN.
}

Oyate woope nica ota Kanan makoce ikiyedan tonwanyanpi. Hena wakağapi nina cekiyapi; qa Israel ohinni tokawicayapi, qa wawicakipi ece. Unkan he tokeca Israel hecen wicakuwapi he. Wakantanka iyawicakta śni he. Ecin iye oie kir anag̉optanpi śni kin, heon iśs eya iyawicakta sni.

Unkan ake oyate woope nicapi Suria ekta Israel oyate etanhan wicinyanna wan wayaka akiyahdapi; qa akicita tancan wan Naaman eciyapi, he tawicu kin wicinyanna kin yuha. Unkan Naaman makośica teliika wan ececa, he lepros eyapi; tancan kin owanca ahinapa. Tuka pejihutawicaśta kin tuwedan wanna asniyan okihi śni.

Unkan wicinyanna wan yuhapi qon he yuhe cin heya okiyaka; Unkitamakocepi kin ekta Wakantanka tawicaśta wan, waayata e he wowayazan kin de asniyan okihi ce, eya. Unkan wicinyanna kin woyake ciqon he Naaman okiyakapi.

Unkan Suria en wicaśtayatapi qon he Naaman heciya; Ekta ya wo, qa Israel wicaśtayatapi kin wowapi wecagge kta ce, eya. Hecen Naaman mazaska, qa mazaskazi, qa wokoyake ko ota yuha ekta ya.

Unkan Eliśa Naaman u kin he nahon, qa Wa- 
kantanka okiye kta e sdonkiya; qa Naaman asniye cinhan Wakantanka ecedan ohoda qeś ecin. Eliśa wowapetokeca econ kin on iye atayedan yatanpi cin śni. Tuka Wakantanka yatanpi qeśs ecin.

Unkan wanna Naaman Kanan en hi. Canpahmihma iyotan waśte wan opa, cy taokiye ota om i. Naaman wijica kin on witantanka, qa Eliśa ota on kicicajuju kinhan, on etanhan kinihan kta kecin. Hecen Eliśa tatiyopa kin en taśunke koya, inajin. Tuka Eliśa tankata inape śni ; tuka Jordan wakpa kin en śakowin akihde ihdujaja wo, kinhan anicisni kta ce, eya okiyag wicaśi.

Unkan heon Naaman śihda, qa heya; Tokeś waayate cin tankan hinape ça, micahda hinajin, qa iye Tawakantanka kin cajehdan mayazan kin en nape on amaputake ça, asnimaye kta nace epce ciqon, eya.

Qa he sihda kin on, Kanan makoce kin en wakpa wanjidan en omihdujaja śni, qa mitamakoce kin ekta e wakpa tanka wanji e en omihdujaja kinhan, iś eya hee on amasni kte, eye ça hda. Tuka taokiye kin e iyakapeye wacinpi, qa heciyapi; Waayate cin taku tehika wanji e econ niši ehan, he ecanon kta he. Tuka de waśakayedan econ niśi, unkan ecanon kte śni, eciyapi.

Unkan Naaman taokiye token eciyapi qon he anawicağoptan; qa Jordan wakpa kin he ekta i, 
qa Eliśa oie kin on, śakowin akihde miniyohpeiçiya. Unkan cehpi kin ekicetu, qa hokśsiyopa iyecen celipi kin panpanna eyaku.

Hehan Naaman hde śni itokam, Eliśa yanke cin ekta e tokaheya i, taokiye kin om i. Qa he

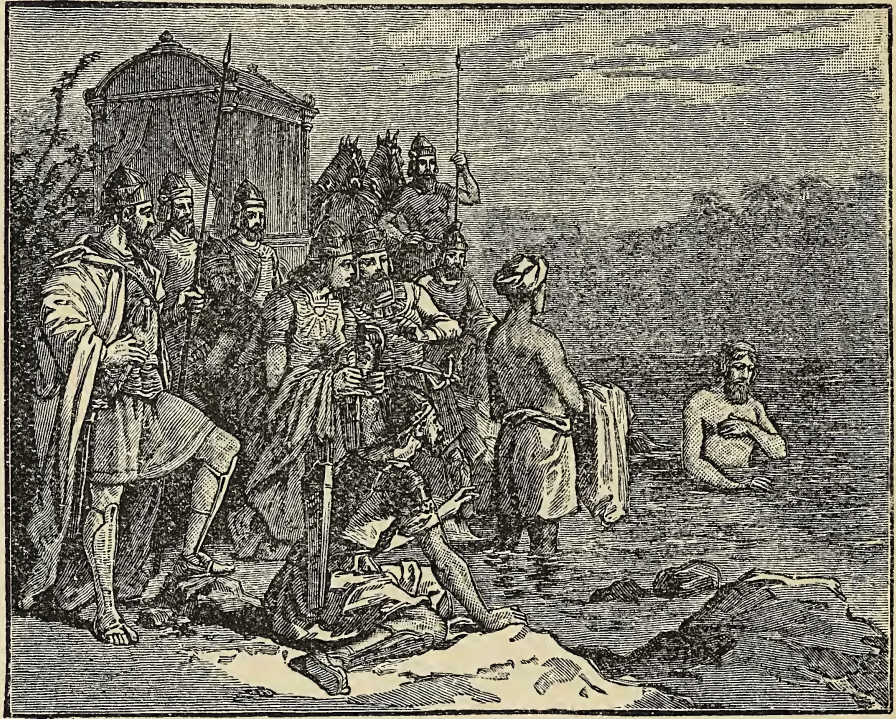

NAAMAN JORDAN EN.

itokam hen ye çehan, wahaniçida ye ciqon he iyecen ye śni. Tuka Eliśa yanke cin en katinyan i, qa heya; Maka akan taku wakan wanica ce; tuka nita Wakantanka hecedan Wakantanka e.sdonwaya ce, eye ça hehan nina pikicida. Qa hehan icimana wakağapi cekiye kte śni, keya. Qa mazaska 
qa wokoyake ko yuha i qon hena Eliśa qu kte hinca. Tuka Eliśa takudan on wokajuju icu cin śni.

Hehan Naaman tacanpahmihma okipe ça tiyatà hda. Unkan Eliśa taokiye wan, Gehazi eciyapi, he Eliśa takudan wokajuju icu cin śni kin he nahon; unkan iye kin token han kinhan taku eihdaku kte cin he awacin, qa itonśni wacin, qa hecen Naaman etapa.

Unkan Naaman Gehazi ahitapa wanhdake çehan, taku toketu nace, ecin. Qa kinajin, qa ipsice ça itkokipe ça, Owasin tanyan unpi he, eya. Unkan Gehazi iś, Owasin tanyan unpi ce, eya. Qa hehan, Itancan mitawa kin u maśi, qa heya ce; Ephraim he kin eciyatanhan kośka nom en mahipi ; hena waayata cincapi ; he mazaska wokoyake ko wicawaqu kta ce; hena maqu kte, eniciya ce, eya.

Unkan Naaman Gehazi itonśni kin he sdonye śni, qa hecen mazaska kida qon etanhan nonpa akihde qu, qa hena wojuha nom en ojuton; qa wokoyake iś tanomnom qu. Qa hehan iye taokiye nom nakun hena kicicaye wicaśi. Qa hecen Gehazi hena om hde ca, ecen tipi wan hocoka tonpi e en hena ehnagwicakiya. Qa hehan ookiye kin kihdewicaya; qa iś hehan ake Eliśa ekta litakini.

Gehazi tohan śce cin Eliśa sdonye śni, qa nakun sdonye kte śni kecin. Tuka tuwe he sdon- 
ya yanka Wakantanka hee; qa Wakantanka Eliśa okiyaka. Qa mazaska kin hena taku on opeton kte cin nakun okiyaka; magia, qa hastanyanka wojupi, qa tatanka, tahinca wanunyanpi, qa wica winyan ookiye ko, hena opeton kte cin he. Tokeś Gehazi hena yuhe cinhan on iyuśkiniçiye kta kecin tuka.

Hehan Eliśa, Tokiyatanhan yaku he, eciya. Unkan iś, Tokidan wai śni ce, eya. Tuka Eliśa heciya; Unkan micante kin nicii śni kecanni he. Qa Naaman wanniyake çehan, ihdamna qa itkonicipe śni he. Ihomeca Naaman wowayazan ecece ciqon, he niś ito enicece kta ce; tohan yani kin hehanyan, qa sanpa nicinca kin koya, eciya.

Unkan itam Gehazi tancan kin owanca wa ska kin iyecekcen ahinapa. Hehan Gehazi tankata inape ça iyaya. Wowayazan kin he tuwe ececa eca oyate wicehna un śni ece.

\section{WOONSPE 34.}

ISRAEL WICAŚTAYATAPI EHAKE KIN.

Eliśa taku wowapetokeca ota econ nayalionpi. Hehan Eliśa ța. Tan zaniyake mahipiya ekta eyakupi śni. Tuka țe ça tancan kin hnakapi. Tuka naği kin e qe mahpiya kin ckta kihda. Eliśa te cin hehan Israel oyate sanpa nina wahtanipi. Qa* 
Israel en wicaśtayatapi ota owecinhan upi ; tuka owasin śicapi, qa wakağapi cekiyapi.

Heon etanhan iś eya Wakantanka sihda, qa Israel oyate kin nina iyopewicaya.

Makoce wan Aśur eciyapi. Unkan makoce kin he en wicaśtayatapi kin he taakicita ota om Kanan en zuya hi; qa Israel oyate wicakize ça wicaktedan, qa Kanan makoce en otonwe kin owasin icu, qa taku yuhapi koya; qa oyate kin owasin wayaka iwicacupi.

Tho, Wakantanka ohodapi śni, qa nina wakihtanipi kin on etanhan kakiświcaye cin hee. Hehan Israel nina wicanihinciya.

Iho, hokśipidan, wicinyanpidan, Wakantanka tocanteptanye kin hanhi, tuka wowitonpe, qa tehika ce. Heon nina ohoda po. Qa nakun iśnana waonśida, waśte ce. Wacinyanpi qa sanpa iye waśte dake niyanpi kta e nina icekiya po.

\section{WOONSPE 35.}

HEZEKIAH WAKANTANKA WACINYE CIQON.

Solomon wahtani qehan, Wakantanka token iyopeye cin he nayahonpi qon. Solomon cinca wan wicaśtayatapi ; tuka wicaśtayatapi tokeca wan Jeroboam eciyapi e Solomon cinca kin makoce tankaya ki, Kanan makoce kin en. Qa Jeroboam 


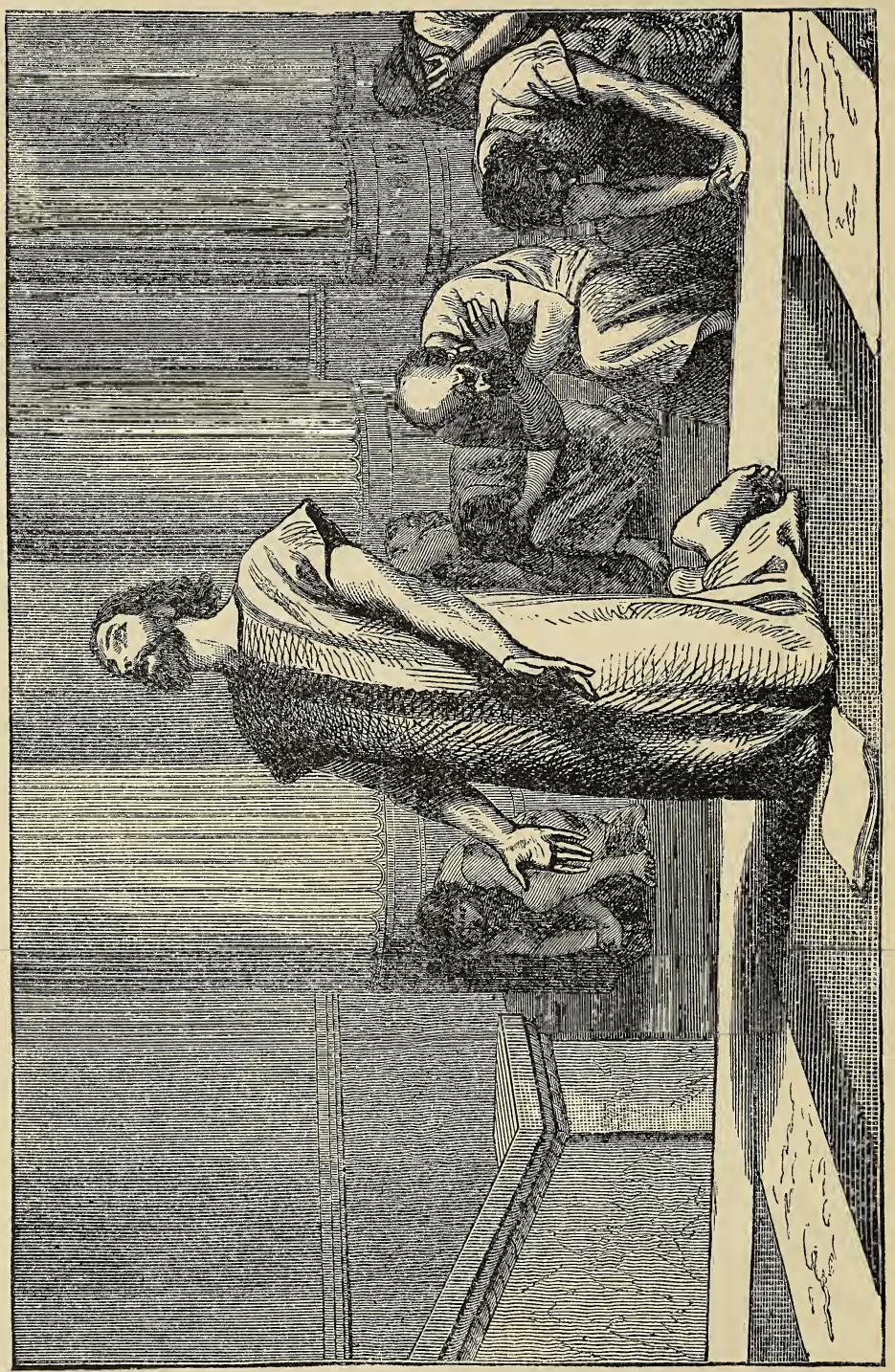


HEZEKIAH WAKAN'TANKA WACINYE CIQON. 151

Israel wicaśtayatapi kin eya cajeyatapi ; qa Solomon cinhintku kin iś Juda wicaśtayatapi eya cajeyatapi. Qa Juda wicaśtayatapi kin he Jerusalem en ti, qa unma kin e iś icunonpa.

Hena țapi, qa hetanhan wicaśtayatapi ota; hena ecen owecinhan unpi. Juda en wicaśtayatapi wanjikji waśtepi, tuka ota śicapi.

Unkan wicaśtayatapi wan Hezekiah eciyapi. Juda oyate en wicaśta kin he waśte, qa Wakantanka wacinyan, qa oyate kin tipi wakan kin en nina Wakantanka ohoda au kta e cinwicakiya.

Aśur wicaśtayatapi wan Israel azuwicaye ça wicaktedan, qa wayaka iwicacu qon. Tuka Wakantanka iyawicakta śni qon hee ake nakun Jerusalem en zuya i ; ozuye tanka i, qa Jerusalem otonwe kin ihdukśanyanpi. Hecen otonwe conkaśke tiyopa owasin sutaya nakitakapi. Heca tuka nakun ozuye kin conkaśke ahiyohpaye kte sece cin he on oyate kin nina wikopapi. Tuka Hezekiah Wakantanka nina wacinye ça ozuye tanka eśta, etanhan niye kte cin he sdonkiya.

Aśur oyate kin Jerusalem ihdukśan yakonpi kin he icunhan nina Wakantanka ośtehdapi.

Unkan anpetu wan en Aśur wicaśtayatapi kin wowapi wan śicaya Hezekiah kicaga. Wowapi kin he kaken kaga: Nitawakantanka kin he niniyan okihi śni ce. Wanna oyate ota ohiwicawaye. Oyate kin hena iś eya tawakantankapi yukan, 
tuka niwicayapi śni: hecen niśs eya Nitawakan-. tanka kin he niniyan okihi śni ce, eciya.

Hezekiah wowapi kin he kaipi, qa yawa. Tuka yawa kapin, sicaya kicag̀e cin heon. Unkan yuha tipi wakan kin mahen i, qa wowapi qon he itokam

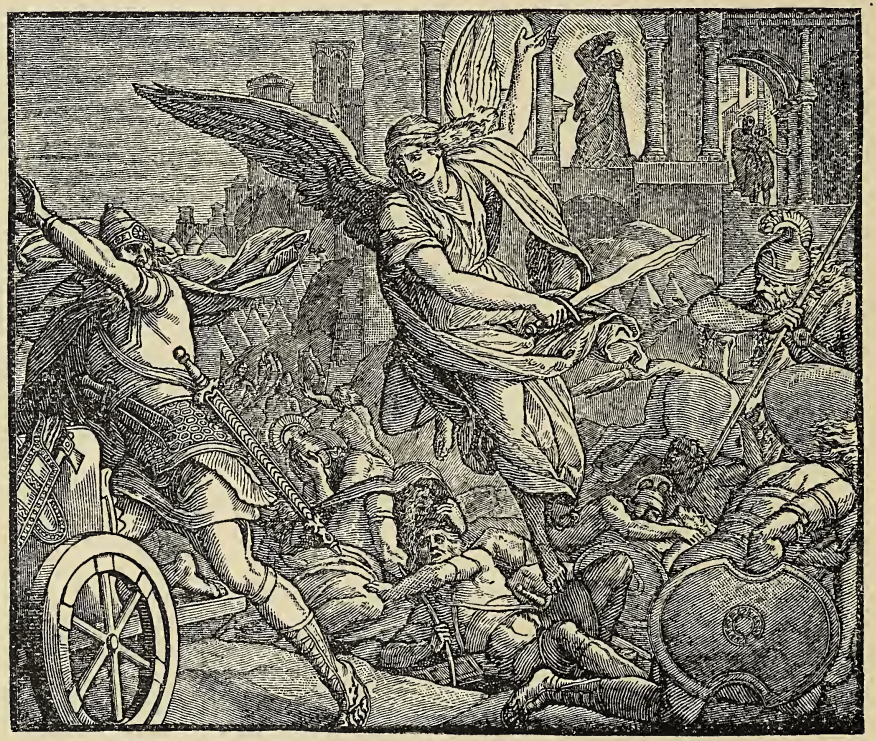

MAḦPIYA OHNIHDE AŚUR WICAKTE.

yumdaya ehpeya. Qa hehan Wakantanka cekiye, qa heya; O Wakantanka, niśnana Wakannitanka, qa iniyotan qon, qa mahpiya maka hena yakagic cin. Tuka wakag̉api kin hena e qe taku śni. Hena can, qa inyan e owicakaggapi ; qa hena oyate waniyanpi okihipi śni. Aśur wicaśtayatapi kin 
de etanhan niunkiya miye. Hecen niśnana Wakannitanka kin he oyate sdonyapi kte, eya.

Hezekiah tawocekiye kin Wakantanka nakicihon. Qa Wakantanka taohnihde wan hanyen en wicai, qa Aśur oyate kin kektopawing̀e opawing ge sanpa kektopawing’e wikcemna śahdoġan sanpa kektopawinge zaptan țewicaya. Hehan tona nipi kin nina yuśinyayapi qa nakipapi. Hecen Wakantanka Hezekiah nikiya, qa heon oyate sdonya-. pi kin on Hezekiah nina pida qa iyuśkin.

\section{WOONSPE 36.}

NEBUKADNEZZAR, QA MAZASKAZI WAKAǴAPI KIN.

Hehan Hezekiah țe cin iyohakam, wicaśtayatapi ota owecinhan Jerusalem en unpi. Tuka ota ścapi, qa Wakantanka ohodapi śni. Qa Jerusalem oyate kin ota iś eya iyewicacecapi, qa wakagapi cekiyapi.

Unkan Wakantanka he śihda, qa waayata tawa en ye si ; qa icimana oyate kin he taku akipapi eśta, ihawicakta kte śni; qa oyate tokeca wan azuyewicaupi kta ; hena waayate cin he owicakiyake si.

Unkan hehan makoce wan itehanyan, Babulon ceiyapi. Unkan hetanhan wicaśtayatapi wan Ne$7^{*}$ 
bukadnezzar eciyapi, he ozuye tanka hduha, Jerusalem otonwe kin takpe hi, qa ihdukśanyanpi, qa ecen unhanketa conkaśke kin eyohpaye ça, tipi waśte ota ideyapi; qa nakun tipi wakan wan waśte hinca e Solomon kagge ciqon he naknn huhnahyapi; qa tipi wakan kin mahen mazaskazi wiyatke, qa petijanjan ihupa, qa taku waśte ota, hen mahen hdepi qon hena icupi. Qa oyate kin iś ota wicaktepi ; qa tona niwicayapi kin hena wayaka iwicacupi. Qa Nebukadnezzar Juda wicaśtayatapi qon he iśta napin pakpe c̣a hehan, niyake aki, qa kaśka hnake c̣a ecen, țeya. Qa tipi wakan kin etanhan taku waśteśte icu qon hena iye totonwe kin en wakagapi tipi wan hde, qa hena en ehde.

Hehan Juda oyate kin iyowicakiśica tanka. Qa Babulon wakpa kin kahda iyotankapi, qa ceyapi. Qa candowankiyapi tawapi kin hena on Psalm ahiyayapi ece qon hena ee wakpa kahda coliwanjica can en otkekiyapi.

Unkan he tokeca e Israel oyate kin wayaka iwicacupi kin Wakantanka iyowinwicakiya he. Iye wakihtanipi kin heon iś eya ihawicakta śni.

Juda oyate wayaka awicakipi kin he wanjikji Wakantanka nina wacinyanpi.

Unkan Nebukadnezzar mazaskazi wakaġapi wan tanka kağa, qa can hanske cin he isanpa hanska kagga, qa tankan hde, qa taoyate kin en 
wicaśta tankapi, qa wayaco, qa akicita tancanpi kin hena wahowicaye ça, wakağapi kin wanyag u

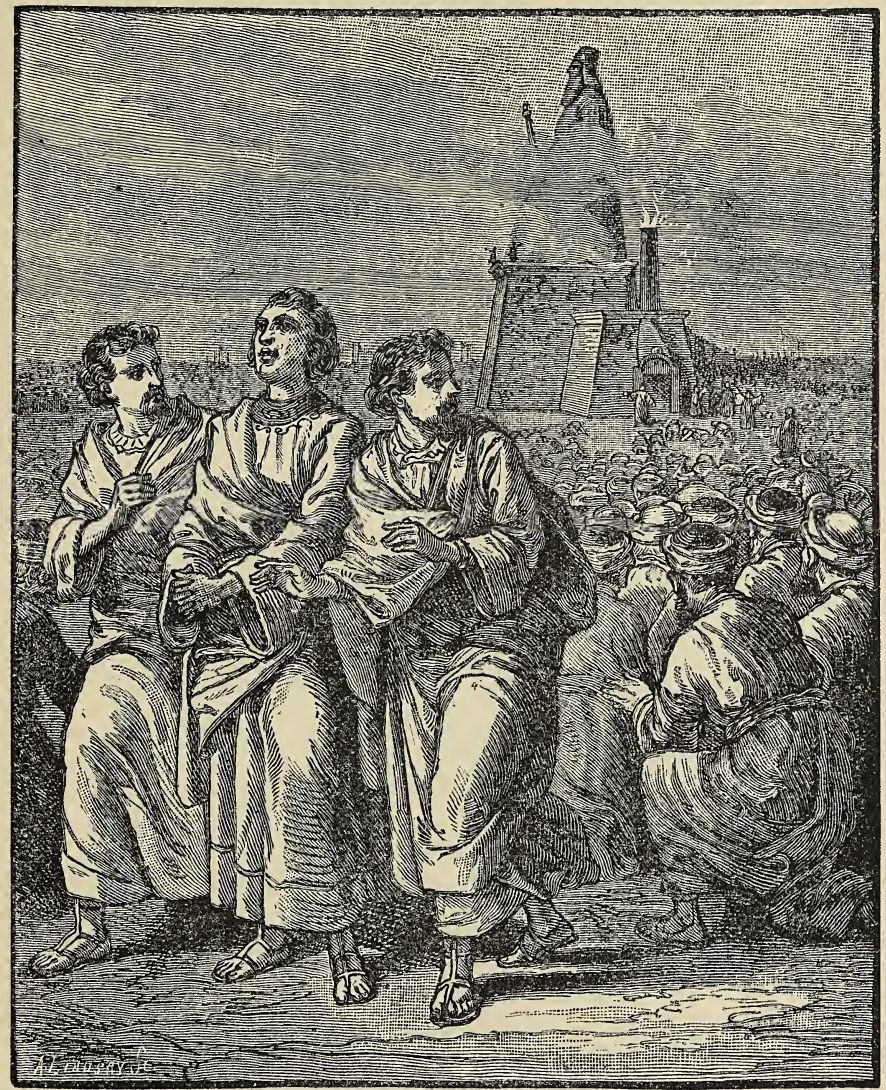

ŚADRAK, MEŚAK, QA ABEDNEGO.

wicaśi. Unkan Nebukadnezzar Juda oyate etanhan kośka waśte yamni wayaco wicakağa, qa he- 
cen hena iś eya en u wicakiya. Śadrak, qa Meśak, qa Abednego, hena eepi.

Unkan wicaśta tokapapi, qa akicita tancan, qa wayaco ko, owasin wakaġapi kin ihdukśan ahinajin kin ; hehan wicaśta wan eyanpaha aya; Oyate kin, tohan cotanka qa candowankiyapi ocaje ota yahotonpi nayahonpi kinhan, owasin makata ehpeniçiyapi, qa wicaśtayatapi wakag̉api wan ehde kin he ohoyadapi kta ce. Qa tuwe ecanonpi śni kinhan, ecahankeya peta ide wan tanka en oiyohpeniyanpi kta ce, eya aya.

Unkan wanna cotanka qa candowankiyapi ocaje ota yahotonpi, hehan oyate kin owasin makata ehpeiçiyapi qa wakaggapi kin he ohodapi.

Unkan wanjikji wicaśtayatapi kin wokiyag ipi qa heyapi; Wicaśtayatapi ohinniyan kin, tohan mazayahotonpi ota hotanin kinhan, oyate owasin makata ehpeiçiyapi, qa wakaġapi eyahde kin he ohodapi kta, keha ci. Qa tuwe econ śni kịhan, peta ide wan tanka en oiyohpeyapi kta kehe ciqon. Kośka yamni nioie kin anağoptanpi śni, qa wakagapi eyahde qon ohodapi śni ce, eya okiyakapi.

Hehan Nebukadnezzar nina sihda, qa kośka kin hena huweye wicaśi. Hecen kośka yamnipi qon en awicahipi. Hehan Nebukadnezzar heya; Wakagapi wan ewahde qon he ohoyadapi sni, keyapi kin he wicakapi he, ewicakiya. Qa hantuqe 
tohan cotanka candowankiyapi ocaje ota hotanin nayahonpi kinhan, makata ehpeiçiyapi qa ohoda po. Tuka ecanonpi śni kinhan peta ide wan tanka en oiyohpeniyanpi kta ce; qa he tuwe Wakantanka ka e minape kin de etanhan niniyanpi okihi kta he, ewicakiya.

Hehan kośka kin iś ayuptapi qa heyapi; Unkitawakantankapi kin he ninape qa nitawakantanka ko etanhan niunkiyapi okihi ce ; qu iś Unkitawakantankapi dehan niunkiyapi kte śni naceca eśta, tohinni nitawakantanka kin he ohoundapi kte śni ce, eciyapi.

Hehan Nebukadnezzar ite ececa śni, qa cante okiçunnica hinca; qa peta qon he śakowin akihde piyah kanye wicaśi. Qa hehan taakicita waśakśakapi ece wicakahnig̉e ça, kośka yamnipi qon hena petan ehpeye wicaśi.

Hecen kośka yamnipi qon hena pawicalitapi, qa tawokoyakepi ko wicakiyuśdokapi śni ecehnana peta nina ide śaśa kin he ehna ehpewicayapi. Unkan peta kin nina kate cin heon wicaśta kin tona he kośka kin petan ehpewicayapi qon hena owasin iyokatapi on țapi.

Kośka kin hena petan ehpewicayapi kin, hehan Nebukadnezzar peta kin ikiyedan inajin, qa huhnagapi kte cin he awanyag nawicajin. Tuka inyun, wicaśta tom peta tanka kin cokaya omanipi ; qa wanji Wakantanka cinhintku kin iyececa, 
he wanyake cin hehan nina yuśinyaya. Qa panyanhan taokiye kin hewicakiya; Wicaśta yamni petan ehpewicunyanpi sece ciqon, eya. Unkan taokiye kin ayuptapi qa, Han, wicaśtayatapi, heconqonpi ce, eyapi. Hehan Nebukadnezzar; Wicaśta tom peta kin cokaya tokeca śni omanipi wanwicamdaka ce, qa wanji Wakantanka cinhintku ee seca ce, eya.

Hehan Nebukadnezzar ikiyedan ye ça, wica-

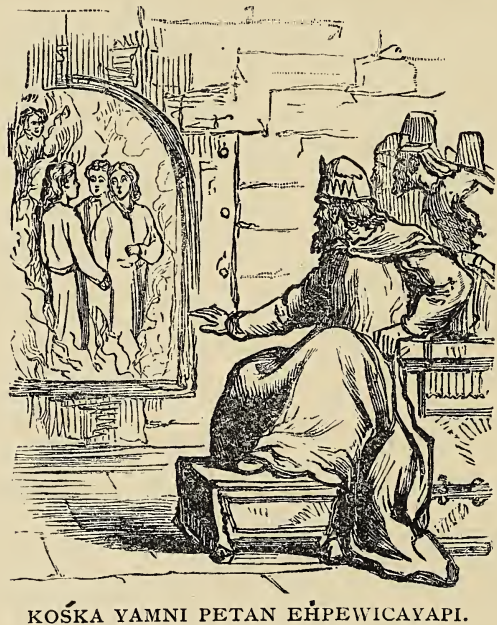

kipan, Śadrak, Meśak, Abednego, Wakantanka iyotan wankantu taokiyepi kin, hdinapa po, qa den ku po, eya. Unkan kośka qon hena eepi e peta tanka kin cokaya tanhan hdinapapi. Hehan wicaśta tokapapi qa akicita tancanpi kin hena en 
au, qa wanwicayakapi; tuka pahinpi qa tawokoyakepi eśta tuktedan gupi śni, qa nakun ecaca gumnapi śni.

Hehan Nebukadnezzar heya; Oyate kin tuwe Śadrak, Meśak, Abednego, Tawakantankapi kin he wicoie wanji ceiye cinhan baśpuśpupi kta ce ; qa tiyohnaka tawa kin nakun ihangyapi kta ce, eya.

Qa hehan Nebukadnezzar kośka yamnipi kin hena hetanhan piya hin itancan wicakaga.

\section{WOONSPE 37.}

NEBUKADNEZZAR WIHAMNA QON.

Nebukadnezzar wanna Wakantanka ohan wanjikji nalion, qa sdonya. Tuka nakun sanpa iye kin watankaiçida, qa witantanka, heon etanhan Wakantanka wihamnakiya he iye decen ohdaka.

Nebukadnezzar miye, tipi iyotan waśte wan ohna ozimiçiye ça, mitohe kin ohna munka icunhan wiwahamna. Unkan wiwahanmde cin de en taku wanmdake cin he nina yuśinyemaye ça kowakipa.

Qehan Babulon en wicašta ksapa owasin wicaweco, qa wiwahanmde cin de mitokam iyukcanwicawakiya; tuka yukcanpi okihipi śni. Unkan owihanketam Daniel en mahi.

Daniel Israel oyate etanhan, Nebukadnezzar 
Jerusalem oyate wayaka awicahdi qon he ehan opa. Qa he ehan nahanliin hokśidan. Tuka wanna wicaśta tanka, qa ksape ça Wakantanka nina wacinyan. Qa Wakantanka iś eya Daniel waśtekidaka.

Unkan wanna Daniel en hi kin, hehan Nebukadnezzar heya; O Daniel, wowihanmde woanalibe kin de omiciyaka ye. He odaka oyakihi e sdonciya ce, eya.

Iho, wiwahamna, unkan inyun, maka kin cokaya, can tanka wan hanska, qa mahpiya kin iyapatoya han. Qa canwapa waśte aicagge ça, waskuyeca ojudan aicağa. He owasin unkitawotepi. Qa can kin he ihukuya ohanzi kin en woteca qeya wankapi qa zitkadan anpehepiya unpi kin iś adetka kin hena en oiyaheyapi ce.

Unkan inyun waawanyaka wakan wan mahpiya eciyatanhan kutkiya u wanmdaka. Unkan he hotankakiya heya; Can kin yucan po, canwapa qa waskuyeca ko hena hnahe kta ce. Qa zitkadan woteca ko, hena tokan iyewicaya po. Qa can adetka kin kaksapi, qa can kin kawanka po; qa can huta kin he okapta po; cu mahpiya kin on spayeye kta ce.

Qa cante kin iś woteca cante wan eckiya canteye kta, waniyetu sakowin hehanyan.

Unkan Daniel he nahon qehan, wihiyayedan oape wanji hehanyan iyukcan; qa he iyukcan 
kin on iyoyaka. Unkan wicaśtayatapi kin heya; Daniel wowihanmde kin on naġiyeiçiye śni wo.

Unkan Daniel iś ayupte ça heya, O wicaśtayatapi itancan mitawa, can wan wandake cin he niye ce. Wicaśtayatapi nitanka, qa maka akan nitowawidake tanka qon he hee ce.

Unkan Iyotan Wankantu Wakantanka he e niyaco ce. Heon winicaśta qon he enicihdaku kta ce. Hecen woteca ehna yaun, qa tatanka iyecen wato e yate kta; Wakantanka e iśnana owancaya Itancan; qa iye token cin eca towawidake kin ecen ecakicon ece kin he sdonyeniye kta, on hecanicon. Heon waniyetu sakowin hehanyan henicece kța ce, eya.

Wicaśtayatapi, mioie kin awacin, qa wayahitani kin elipeye ça, wicoowotanna e econ wo, qa wahpanicapi kin waonśida wo ; okinni hecanon kinhan Wakantanka wiconi niçu nun ce, eciya.

Daniel hena hecen eciya, tuka nakun iyowinye śni watankaic̣ida kin he ayuśtan śni. Unkan etanhan omaka wanji, hehan Nebukadnezzar tipi iyotan waśte wan oti qon he ohna omani, qa heya; Babulon otonwe wakage de ehan qon tanka śni he. Mitookitanin qa mitowaśake kin on dena owakihi.

Nebukadnezzar hena heye cin, he icunhan mahpiya kin eciyatanhan wicaho wan ahinhipaya, qa heciya ; Nebukadnezzar wicaśtayatapi he niye ci- 
ca ce, nitowawidake qon he enicihdakupi ce, eya.

Unkan wicaho wan nahon qon etanhan wihiyayedan oape hanke hehanna, taku kin dena owasin Nebukadnezzar hiyahde. Qa hecen tawacin qon iye tantanhan kahintapi. Hecen tatanka iyecen peji ece yuta. qa cehpi kin iś cu mahpiya kin on spaya, qa tancan kin owanca wanmdi hin kin iyecen hin uya; qa śake kin iś zitkadan śake iyecen uya, waniyetu śakowin hehanyan.

Unkan waniyetu śakowin iyehan i kin, hehan Nebukadnezzar tawacin qon kiyohpaya. Unkan iśta yuwankan ehdaku, qa malipiya kin ekta etonwan, Wakantanka yatan qa yaonihan.

Unkan taakicita qa itancanwicakiye ciqon hena, wanna tawacin ekicetu wanyakapi qehan, owasin en ipi, qa ake piya hin wicaśtayatapi kicag̉api. Unkan hehan wicaśtayatapi waśte hinca qa heya; Iho, Nebukadnezzar miye ce, mahpiya Wakantanka kin mdatan qa mdaonihan ce. Tuwe ohinni wowicake econ kin he hee ce; qa tawoyaco kin ohinniyan ce. Qa tona wahaniçida omanipi keś, iś he icunhan wicayuśsihtin okihi ce, eya. 
BELŚAZZAR, QA WICANAPE WOWA QON. 163

\section{WOONSPE 38.}

BELŚAZZAR, QA WICANAPE WOWA QON.

- Nebukadnezzar te cin, hehan ake wicaśtayatapi wan tokeca, he Belśazzar eciyapi. Nebukadnezzar he takojakpaku ; tuka iś eya ake Nebukadnezzar ohan kin iyececa, wahaniçida, qa nakun wakaġapi cekiya. Unkan Belśazzar anpetu wan en wonwicaya, qa wicaśta wijicapi, qa wicaśta tankapi, wicaśta itancanpi ko wicota wicakico, qa hena wicitokam miniśa yatkan.

Jerusalem en tipi wakan kin hetanhan Nebukadnezzar mazaskazi wiyatke, qa taku waśte ota icu, qa hena iye totonwe kin en wakagapi tipi wan hde, qa hena en ehde nayahonpi qon.

Unkan Belśazzar wonwicaye cin he icunhan wiyatke kin hena huweye wicaśi. Qa iye tawicu wicaye cin hena, qa itancan wicayuhe cin hena ko om, wiyatke kin hena ohna miniśa yatkanpi. Qa miniśa yatkanpi kin he icunhan, wakaggapi wicahdepi kin hena nina wicahdatanpi.

Belśazzar Wakantanka heceedan wakan, qa ohan wanjikji nahon tuka ohoda sni kin he Wakantanka śihda. Qa Belśazzar Wakantanka ohan wanji sdonya.

Wicaśtayatapi ti ohna miniśa yatkanpi kin he icunhan, petijanjan ihupa he cin iwankam, tiona- 


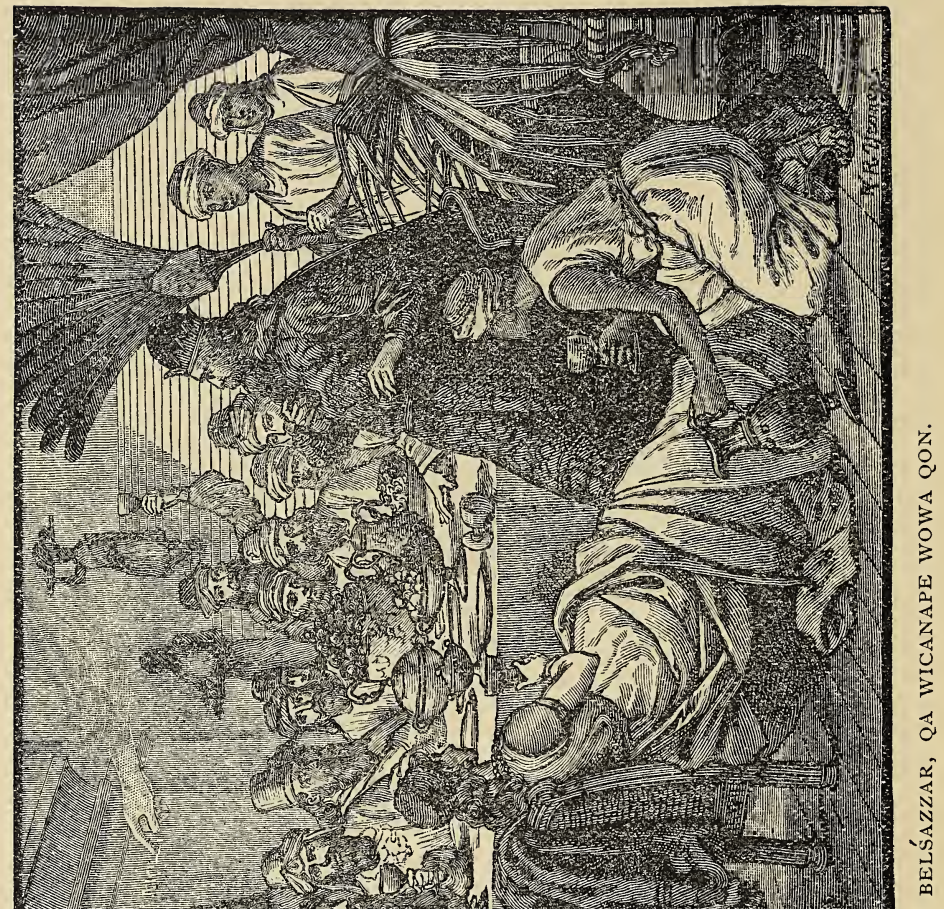


BELŚSAZZAR, QA WICANAPE WOWA QON. 165

ptan kin en wicanape wan wowa, wicaśtayatapi kin wanyaka. Wicaśta e wanyake śni, tuka wicanape wan ecedan wowa e wanyaka. Hehan wicaśtayatapi kin nina yuśinyaya. Nina inihan kin on cancan, qa hupahu kin napin iciyapa.

Hehan wicaśtayatapi kin panyanhan heya; Babulon en wicaśta ksapapi kin owasin awicau po, eya. Qa en ahi kin, hehan hewicakiya; Tuwe oowa kin de yawa, qa token kapi oyake cinhan, wokoyake duta koyake ça, mazaskazi wanap̣in wan napin kta, qa wicaśtayatapi en wiciyamni kta ce, eya.

Oowa kin he wicoie topa, tuka oyate kin tuwedan yawa okihi śni. Hehan iyotan Belśazzar nina inihan, qa ite kin nina iyoyaka; qa itancan wicakiye cin nakun nina inihanpi.

Unkan Belśazzar hunku wan wicaśtayatapi winohinca hee, hehan nalion qehan, wicaśtayatapi yanke cin en hi, qa heya; Wicaśtayatapi ohinniyan kin, ihnuhan nina ininihan kin. Babulon otonwe kin de en wicaśta wan ksapa un ce, Daniel eciyapi ce; he wahoyaye cinhan hi, qa taku kin de token kapi kin sdonye kta ce, eya.

Unkan wanna Daniel wicaśtayatapi kin en aipi, hehan wicastayatapi kin heya; Daniel he niye he, Juda oyate wayaka awicahdipi qon he enitanhan he. Iho, wanna wicaśta wokcanpi kin 
owasin oowa kin de yawapi qa toketu kin oyakapi kta, tuka tuwedan okihi śni ce.

Tuka niś taku dececa keśs odaka oyakihi keyapi. Oowa kin de dawa, qa toketu kin odake cinhan, wokoyake duta koniyake ça, mazaskazi wanapin wan nayapin kta ce; qa wicaśtayatapi en wiciniyamni kta ce, eya.

Hehan Daniel ayupte ça heya; Wokajuju e qe takudan wacin śni ce; tuka oowa kin de mdawa, qa toketu kin omdake kta ce, eya.

Hehan Daniel wicaśtayatapi kin heciya; Wakantanka canniyeniyan ce; wahanniçida qa Wakantanka tipi wakan tawa kin etanhan wiyatke qeya on miniśa datke ca, nakun Wakantanka ecedan wakan kin he nayahon, tuka ohoyada śni, qa wakaggapi qeya e nina wicadatan kin hena Wakantanka sinhda ce. Qa wowa kin he kaken ka ce; Wicastaniyatapi qon he wanna henakeca kta ce; oyate tokeca wan azuniye ça niktedan, qa makoce nici kta ce, eciya.

Hehan Belśazzar Daniel wokoyake duta koyagye ça, mazaskazi wanapin wan napinkiye ça, oyate kin en wicaśtayatapi wiciyamni kag̉a.

Unkan ihtayetu hepiya wicastayatapi tokeca wan, ozuye tanka hduha, Babulon otonwe kin iyohpaye ça, Belśazzar wicaśtayatapi qon ktepi, qa makoce kin he ohiya. Hecen Wakantanka token eye ciqon owasin iyecetu. 


\section{WOONSPE 39.}

DANIEL, QA MNAJA OTIPI KIN.

Wicaśtayatapi wan Belśazzar kte, qa tamakoce owasin ohiye ciqon he Darius eciyapi. Tuka iś eya ake wakagapi cekiya. Tuka hehan nakun Daniel waśtedake ça, oyate en wicaśta itancanpi, qa wayacopi ko, wiciwankam Daniel itancan kağa ; qa oyate kin owasin Daniel owe unpi kta, kewicakiya.

Daniel wicasta ksape hinca, qa Wakantanka nina wacinyan.

Unkan wicaśta itancanpi kin wicota Daniel sicedakapi, winakiwizipi kin heon. Hecen hena Qain iyececapi. Qain sunkaku Abel winakiwizi. Qa nakun Joseph hunkawanjinwicaye cin hena iyewicacecapi. Qa nakun Saul iyececapi; Saul Dawid winakiwizi.

Qa wicaśta tokapapi kin hena tawacin śicapi, qa Daniel wowiśtece wanji iyaonpe wacinpi. Tuka ohan wanjidan ektaśniyan iyeyapi śni. Tuka nakun nina wiyaonpe wacinpi; qa token econpi kte cin he ihdukcanpi.

Unkan Daniel ohinniyan Wakantanka nina cekiye cin he sdonyapi. Unkan heon etanhan wican stayatapi kin en ipi, qa woope wan kah śipi. Qa 
heyapi ; Anpetu wikcemna yamni hehanyan tuwedan taku wakan tokeca cekiye kte śni ce; tuka niśnana ceniciyapi kta; qa he iyokpani tuwe taku tokeca cekiye cinhan, mnaja tipi en oiyohpeyapi kta ce, eyapi.

Daniel nina Wakantanka cekiye cin, heon heyapi kin wicaśtayatapi kin he sdonye cinhan, okinni woope kin he kagge kte cin itonpe kta naceca tuka.

Unkan hecen wicaśtayatapi kin woope kin he ecen kağe ça ohna yusuta.

Unkan Daniel woope kin he nation; unkan anpetu wikcemna yamni hehanyan Wakantanka cekiye kte śni kin he nina tehike daka.

Daniel anpetu otoiyohi yamni wocekiye eya ece. Qa tipi ohna cekiye cin he en tohan cekiya eca, owanyeye kin he yuzamni hde, qa cekiya ece. Unkan he token on hecon hwo, ecannipi kta nace.

Jerusalem otonwe kin he ekta itoheya etonwan cekiya ece kin heon hecon. Jerusalem en tipi wakan he çehan he ohna Wakantanka taniniçiya ece; qa Wakantanka he waśtedake cin, Daniel he sdonya, heon he tohan cekiya eca, ekta etonwan cekiye cin hecon.

Unkan Daniel wocekiye eya ece qon hecetu wanjica ccon. Anpetu otoiyohi yamni canpeśka makehde inajin, qa Wakantanka onśikida qa tan- 
yan hduhe cin hena on wopida eya ece. Unkan winakiwizipi qon hena eepi e Daniel wocekiye eye cin atayedan wanyag ipi ; qa hehan wicaśtayatapi kin okiyag ipi.

Qa heyapi ; Anpetu wikcemna yamni hehanyan tuwedan taku tokeca cekiye kte śni, tuka niśnana ceniciyapi kta; qa tuwe he iyokpani taku tokeca cekiye cinhan, mnaja tipi kin en oiyohpeyapi kta, woope wan yakağa ci, eyapi.

Unkan wicaśtayatapi kin, Han, he hecetu ce, qa woope kin he mdutokeca kte śni ce, eya.

Hehan heyapi; Daniel Jerusalem etanhan wayaka ahdipi qon hee eca iyae cin onipe śni, qa woope qon he ope śni, anpetu otoiyohi yamni akihde iye Tawakantanka kin cekiya ce, eyapi.

Hehan wicaśtayatapi kin nina icanśica, qa token econ qa Daniel niye kte cin he iyukcan, tuka token econ kta wanjidan iyeye śni.

Hehan ake wicaśtayatapi kin en ipi qa heciyapi; Woope yakagie cin wanna hecetu e dusuta, hecen dutokeca kte śni, eyapi.

Hecen wicaśtayatapi kin Daniel mnaja tipi en oiyolipeye wicaśi. Mnaja kin he makolidoka otipi, qa ohinni wotektehdapi, qa litayetu aya eca, hehan iyotan nina woda hotonpi ece. Hecen Daniel hen oiyohpeyapi kinhan ecahankeya temya iyeyapi kta iyececa.

Unkan wicaśtayatapi kin Daniel heciya; Nita- 


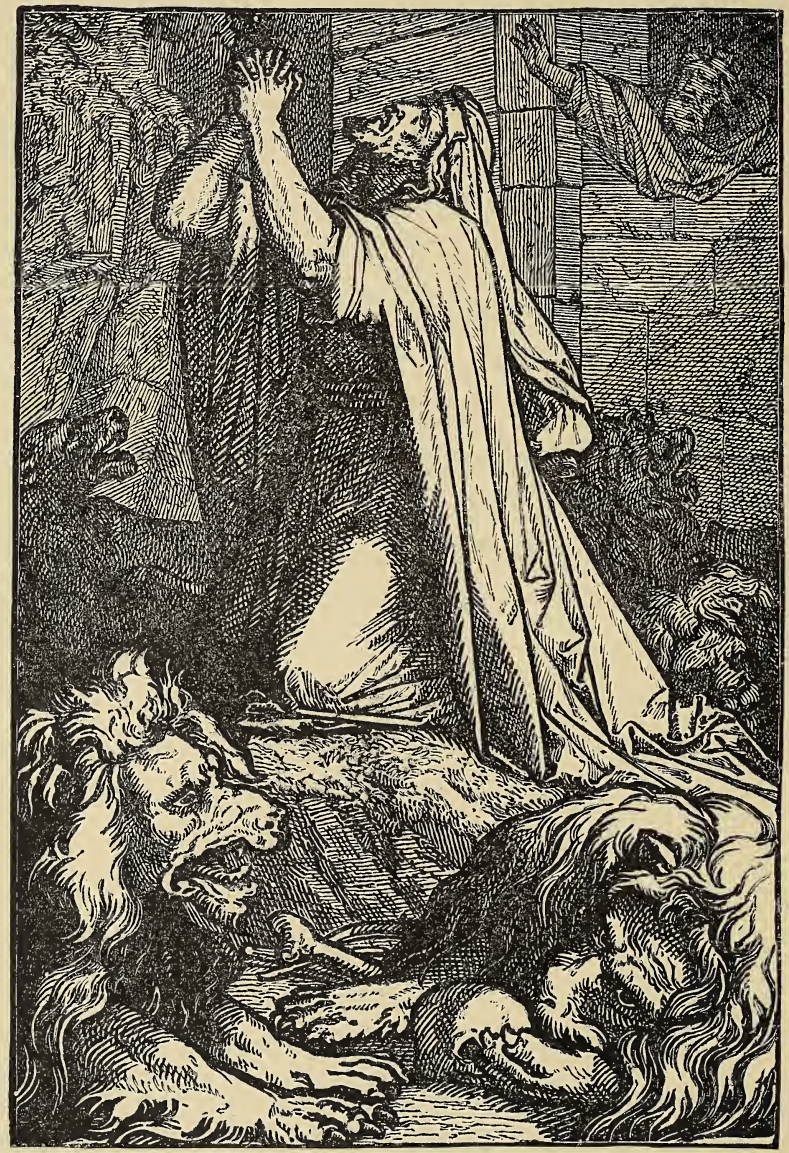

DANIEL MNAJA TIPI EN.

wakantanka heceedan iyotan ce; he niniyan okihi ce, eya.

Daniel mnaja tipi en oiyolipeyapi, qa hehan 
inyan wan en wicaśtayatapi wipuspe tawa wan askamye ça, hehan tona Daniel kipajinpi qon hena inyan kin he on ohdoka kin iyonatag wicaśi.

Wicaśtayatapi woope kagge cin he hdutokeca cin śni e oyate kin he sdonyapi cin kin heon hecon. Qa okinni tuwe Daniel mnaja tipi kin he etanhan eyaku eśta, hecen iye iyaonpapi kte śni, cin kin hena on hecon.

Hehan wicaśtayatapi kin hanyetu kin he Daniel on nina iyokiśsica, qa wote śni, qa taokiye kin magagayapi kta tuka iyokipi śni. Qa hanyetu kin he iśtinbe śni ecen hanlianna hin mnaja tipi kin ekta i.

Mnaja tipi kin en inajin, qa hehan ho onśiic̣iya Daniel kipan qa heya; O Daniel Wakantanka ni un kin taokiye kin, Nitawakantanka ohinni niniye ciqon ake dehan niniyan he, eya.

Unkan Daniel iś ayupte ça heya niyan, $\mathrm{O}$ wicaśtayatapi ohinniyan kin Mitawakantanka iye taohnihde wan u si, qa mnaja i kin owasin nawicatake ça, hecen takudan tokamiconpi śni ce. Tuka ecin takudan śica ecamon śni kin heon etanhan, eya.

Hehan wicaśtayatapi qon nina pida, qa hecen Daniel mnaja tipi kin etanhan icu wicaśi. Qa icupi, tuka tuktedan tokeca sni.

Unkan wicaśta kin tona he Daniel kipajinpi, qa on etanhan mnaja tipi en ehpeyapi kin he 
kaggapi kin hena wicaśtayatapi kin nina canniyewicaya, qa hecen wicaśta kin hena owasin tawicu, cincapi ko om iś ito mnaja tipi kin en oiyohpeye wicaśi.

Hecen hena owasin mnaja tipi kin en oiyohpewicayapi, tuka sicuta ihpayapi śni ecen tancan huhu ko owasin iyahuh temya iyewicayapi.

Hehan Darius wicaśtayatapi kin woope wan kağa ça, makoce kin owancaya yuotanin, qa heya; Oyate kin owasin Daniel Tawakantanka kin hecedan kokipapi kta ce; hecedan Wakantanka kin heon; qa towawidake kin tohinni jujuwahe kte śni ce, eya.

Daniel Wakantanka ecedan waśtedake cin heon wiconte ikope śni kin de nayahonpi. Tona cante ocowasin on Wakantanka waśtedakapi kin hena hececapi kte. Kinhan iś eya Wakantanka hena owilhanke wanin wicakiksuye kta.

\section{WOONSPE 40.}

JERUSALEM AKE EN AHDI KIN.

Israel oyate kin Babulon ekta awicakipi kin; hehantanhan ohinni nina iyokiśicapi; qa, tokin unkitamakocepi kin ekta ake unkipi qeś, ecinpi kin he ota.

Пe itokam wanakaja qehan, Wakantanka Israel 
oyate kin he ake anpetu wan en iye tamakocepi kin ekta ki wicaye kta keya. Wakantanka token eya eca tohinni akiktonje śni.

Unkan ake Babulon en wicaśtayatapi wan tokeca, he Kuros eciyapi. Kuros iś eya wakagiapi cekiyapi nahon icagga. Tuka hehan Daniel Tawakantanka heceedan Wakan kin he nahon qa wicada. Hecen Darius qa Kuros henaos wicastayatapi unpi. Unkan Wakantanka Kuros hecen cante yuskiya; qa Kuros Israel oyate kin iye tamakocepi kin ekta hdapi, qa ake Jerusalem en tipi wakan wanji kicağapi kta, kewicakiva.

Qa hehan Jerusalem en tipi wakan kin etanhan Nebukadnezzar mazaska qa mazaskazi wiyatke, qa taku waśte ota aki qon hena ee owasin Kuros Israel oyate wicakicu.

Qa Babulon oyate kin wicota Israel wawicaqupi, śuktanka, qa śonśonna, qa kamel ko wicaqupi, qa hena waqinwicakiya hdapi.

Hehan Israel oyate kin nina wiyuśkinpi; Babulon otonwe śce cin he wanna elipeya hdapi kin heon. Tuka hehan Jerusalem ekta kipi kin hehan ake iś otonwe kin oiyokiśinya wanka wanhdakapi. Conkaśke kin hee, tuka jujuwaheya wanke ça, hehan tipi waśte ota hulinahya hiyeya. Hececa tuka Israel oyate nina Wakantanka wopida eciyapi; Wakantanka hee ake heci hdiwicaye cin heon. 
Qa Jerusalem en wahna wośnapi wan ehdepi, qa he akan woteca ota Wakantanka wakiyuśnapi, qa nina wopida eyapi.

Qa ake tipi wakan wanji kohanna kag̉api cinpi. Qa hecen can iyotan waśte on kağapi kte cin hena kohanna ihnipi.

Hehan wanna Jerusalem en paha tehanwankantu wan akan tipi ahdehe kte cin inyan kin wanna tokaheya ehdepi kin, hehan oyate kin owasin en kawitaya iheya. Qa wośna itancan kin wokoyake ska koyake ça, cotanka wan yuha, qa hehan dowanpi kin owasin iś candowankiyapi on Psalm ahiyayapi ; kaken eyapi, Jehowa waśte, qa towaonśida kin he owihanke wanica ce.

Hehan wośna itancan kin cotanka kin yajo, qa dowanpi kin owasin Psalm ahiyayapi, qa oyate kin wiciyuśkin on panyanhan iyakiśapi.

- Unkan oyate wiciyuśkin kin he en icunhan, tona wanna wicaśta tankapike cin hena ceyapi; tipi wakan wan tokaheya Solomon kagge ciqon he ehan henana he wanyakapi, qa he kiksuyapi, qa oyate kin Wakantanka onśiwicada, tuka wakilitanipi ece kin hena on he ceyápi.

Unkan hecen wiciyuśkin, qa panyanhan iyakiśapi, qa wanjikji iś icunhan ceyapi ko oqo kin tehan tanhan tanin.

Unkan Israel oyate waniyetu ota tipi wakan kin he kağapi qa ecen yuśtanpi. 'Tuka Solomon 
tipi wakan wan waśte kag̉e ciqon he iyecece śni, qa nakun Wakantanka en taniniçiye śni.

Hehan Israel oyate kin wakağapi cekiyapi ece kin he ayuśtanpi, tuka cante ocowasin on Wakantanka waśtedakapi śni; qa hecen nakun nina wa-

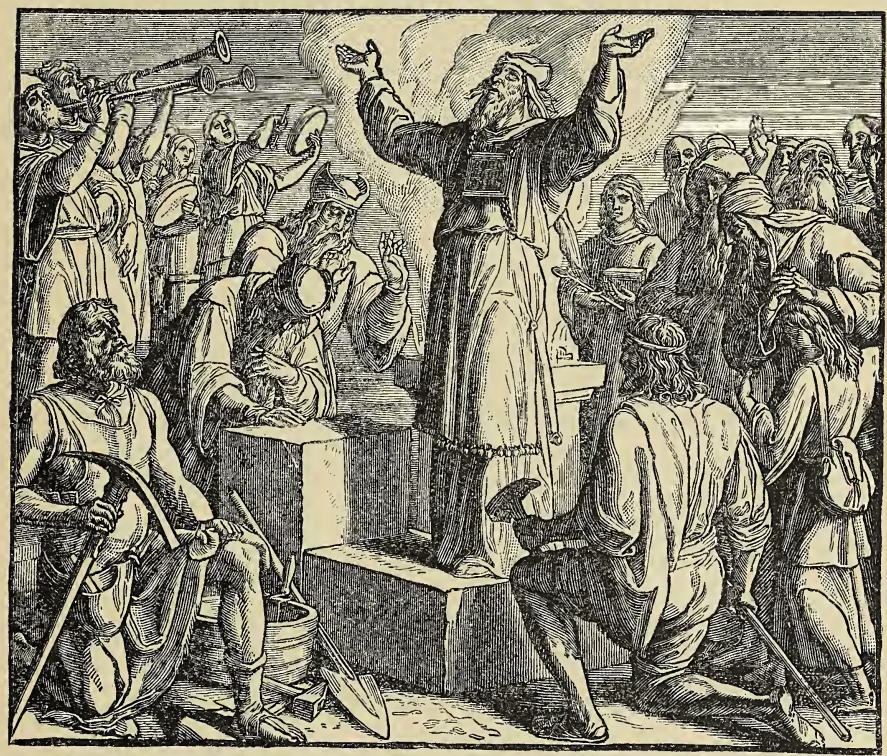

TIPI WAKAN PIYA EHDEPI.

kilitanipi. Tuka he en opeya wanjikji Wakantanka nina wacinyanpi.

Israel oyate Kanan makoce kin en waniyetu ota ounyanpi; qa Wakantanka waayata tawa wanjikji en ye wicaśi, qa hena wahokonwicakiyapi 
ece. Qa Wakantanka Abraham wahoye ciqon he iwahokonwicakiyapi; maka akan Wanikiya wan tonpi kta, kinhan he oyate kin wanikiye kte cin hee kta.

Qa nakun Wakantanka Dawid hecen iwahoya; Dawid tawicoicage wicoicagge owecinhan $\mathrm{u}$ kin hetanhan wanji e Wanikiya wan icag̉e kta.

Unkan Israel oyate wanjikji he nina awacinpi sa, qa Wanikiya wan maka akan hi kte cin he nina cantokpanipi.

Wanikiya wan tonpi kte cin he Bethlehem otonwe kin hetu kta; waayatapi kin he oyakapi, qa oyate kin nalionpi.

Wanikiya kin he Wakantanka Cinhintku kin hee. Jesus Wanikiya maka kin de akan token hi, qa token icag̉e cin he wanna Wowapi Wakan etanhan dawapi qa nayalionpi.

Maka kağapi śni he itokam Wakantanka iye Cinhintku kin maka kin de akan țe u śi. Unkan Cinhintku kin Atkuku oie kin kiciyuśtan kta e maka kin de akan te hi.

Adam wicaśta tokaheya kin wahtani kin heon, qa miś wawahtani qa niśs wayahtanipi kin hena on Jesus Messiya țe hi, qa ake malipiya kin ekta Atkuku etapa kin en kiyotanka; qa ake anpetu ihankeya kinhan u kta, qa tona Jesus waśte dakapi, qa iye tacanku omanipi qon hena wanna wicahduha qa hena om u kta. 
Jesus Messiya towaonśida tanka qon otoiyohi unkicipi un nunwe.

\section{ITANCAN TAWOCEKIYE KIN.}

MATT. 6:9; QA LUK. 11:2.

Ateunyanpi Mahpiya ekta nanke cin, Nicaje wakandapi kte: Nitokiconze u kte.

Nitawacin maka akan econpi nunwe: mahipiya ekta iyececa.

Anpetu kin de anpetu woyute unqu po.

Qa waunhtanipi unkicicajuju miye ; tona śicaya ecaunkicunpi wicunkicicajujupi kin iyececa.

Qa taku wawiyutan en unkayapi śni ye; tuka taku sice cin etanhan eunyaku po.

Wokiconze kin he Niye nitawa, qa wowaśake kin, qa wowitan kin, owihanke wanica. Amen.

\section{WOAHOPE WIKCEMNA KIN.}

1. Mitokam Taku Wakan tokeca duhe kte śni.

2. Wakaġapi kin takudan nic̣icag̉e kte śni, taku wankan mahpiya kin ekta, qa taku kuya maka akan, qa taku maka kin ihukuya mini mahen iyacinpi takudan yakagge kte sni; hena itokam canpeśka makehde inayajin kte sni qa ohoyada kte śni. 
Jehowa Wakantanka nitawa kin he miye, Taku Wakan winawizi waun, ateyapi wahtanipi kin hena cincapi kin en awicawai ece, wicoicage iyamni qa itopa hehanyan, tona śicemadapi kin; qa tona waśtemadapi qa mitawoahope yuhapi kin hena kektopawing hehanyan onśiwicawakida ece.

3. Jehowa Wakantanka nitawa caje kin he ikcekceya cajedate kte sni: tuwe ikcekceya cajeyate cinhan he sicaya econ Jehowa ecankin kta.

4. Anpetu okihpapi kin he kiksuye ça wakanda wo: Anpetu śakpe litayani qa nitolitani owasin ecanon kta; tuka anpetu iśakowin kin he anpetu okih papi, Jehowa Nitawakantanka kin he tawa; he en wicohtani takudan ecanon kte śni, niye, qa nicinkśi, nicunkśi, wicaśta nitaokiye, winyan nitaokiye, nitawoteca qa wicaśta tokeca nitatiyopa en un kin.

Anpetu śakpe en Jehowa mahpiya, maka qa miniwanca, qa taku ohnaka koya owasin kaga, unkan anpetu iśakowin kin okilipa; heon Jehowa anpetu okihpapi kin hdawaśte qa hduwakan.

5. Niyate nihun kici wicahduonihan wo: hecetu kinhan makoce Jehowa Nitawakan tanka nic̣u kin he en tehan yani kta ce.

6. Tuwedan yakte kte śni.

7. Wawicihahapi ecanon kte śni. 
8. Wamayanon kte śni.

9. Nitakoda on takudan oitonśni daotanin kte śni.

10. Nitakoda ti kin cantiheyaye kte sni ; nitakoda tawicu, wicaśta taokiye qa winyan taokiye, tatanka tawa, taśunke, qa taku nitakoda tawa kin takudan cantiheyaye kte sni.

\section{WICAYAWAŚTEPI KIN.}

MATT. 5:2-10.

Unkan i hdukawa qa waonspewicakiye ca heya :

1. Tona cante mahen onśiiçidapi kin hena wicayawaśtepi ce; mahpiya wokiconze kin he tawapi ece.

2. Tona ceyapi kin hena wicayawaśtepi; hena wicakihnapi kta.

3. Tona onśihianpi kin hena wicayawaśtepi; maka kin de tawapi kta.

4. Tona woowotanna on wotektehdapi qa ipuzapi kin hena wicayawaśtepi ce; hena imnanpi kta.

5. Tona waonśidapi kin hena wicayawaśtepi ; hena onśiwicadapi kta.

6. Tona cante en ecepidan kin hena wicayawaśtepi ; hena Wakantanka wanyakapi kta. 
7. Tona wookiye kagapi kin hena wicayawaśtepi ; hena Wakantanka cinca ewicakiyapi kta.

8. Tona woowotanna econpi kin on ścaya kuwapi kin hena wicayawaśtepi ; mahipiya wokiconze kin he tawapi ece.

9. Tohan aniiapi qa śicaya nicuwapi, qa miye on itonśniyan taku śica owasin eniciyapi kinhan, niyawaśtepi ece.

10. Wiyuśkinpi qa nina cante waśte po, mahipiya ekta ocinyopeniyanpi tanka yanka; wicaśta wokcan nitokam unpi qon hena iś eya hecen wicakuwapi qon. 






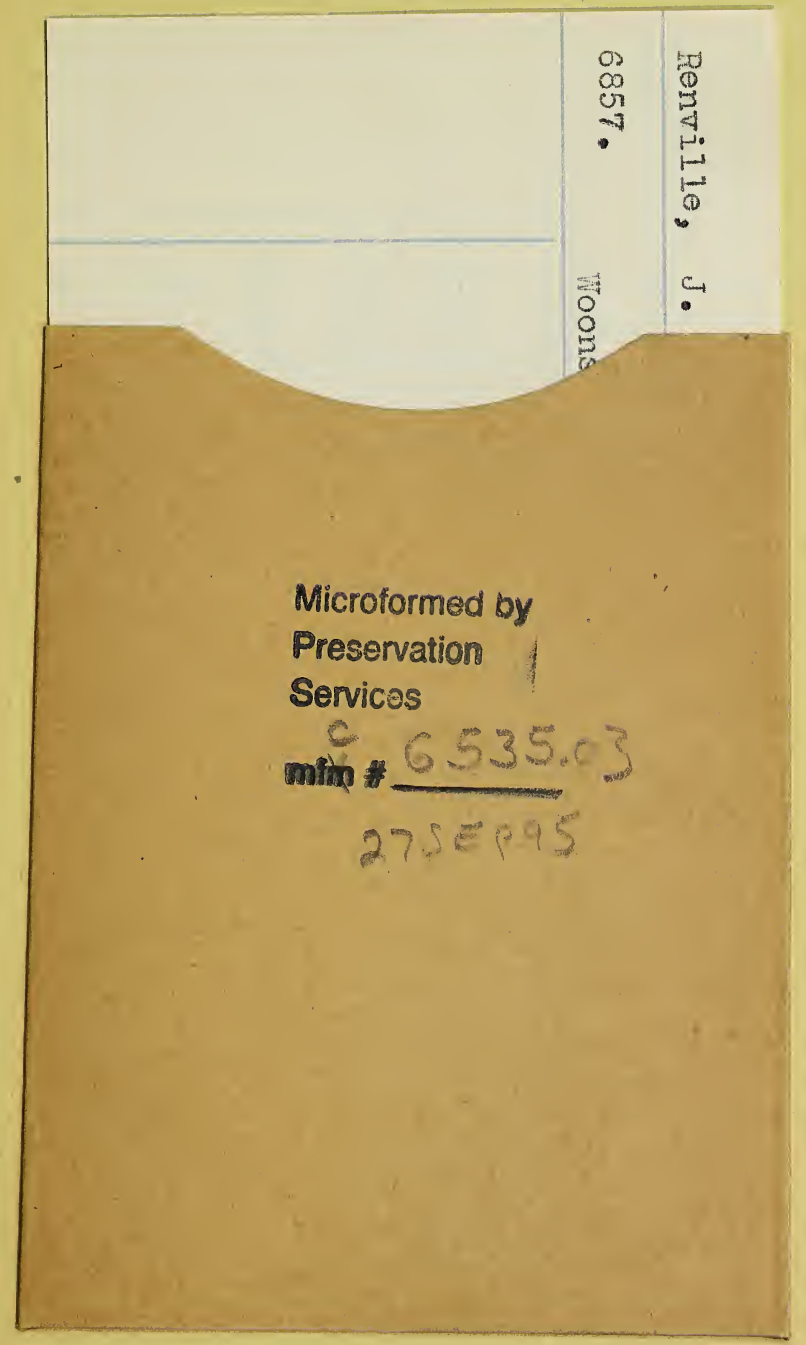


SMITHSONIAN INSTITUTION LIBRARIES

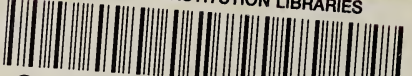
3908800578 (I) 39088005784806 